ESAIM: M2AN 48 (2014) 1639-1679

DOI: $10.1051 / \mathrm{m} 2 \mathrm{an} / 2014015$
ESAIM: Mathematical Modelling and Numerical Analysis

www.esaim-m2an.org

\title{
STUDY OF A LOW MACH NUCLEAR CORE MODEL FOR TWO-PHASE FLOWS WITH PHASE TRANSITION I: STIFFENED GAS LAW
}

\author{
Manuel Bernard ${ }^{1}$, Stéphane Dellacherie $^{2}$, Gloria Faccanoni ${ }^{3}$, \\ BÉrÉnice Grec ${ }^{4}$ And Yohan Penel ${ }^{5}$
}

\begin{abstract}
In this paper, we are interested in modelling the flow of the coolant (water) in a nuclear reactor core. To this end, we use a monodimensional low Mach number model supplemented with the stiffened gas law. We take into account potential phase transitions by a single equation of state which describes both pure and mixture phases. In some particular cases, we give analytical steady and/or unsteady solutions which provide qualitative information about the flow. In the second part of the paper, we introduce two variants of a numerical scheme based on the method of characteristics to simulate this model. We study and verify numerically the properties of these schemes. We finally present numerical simulations of a loss of flow accident (LOFA) induced by a coolant pump trip event.
\end{abstract}

Mathematics Subject Classification. 35Q35, 35Q79, 65M25, 76T10.

Received November 11, 2012. Revised Ferbuary 11, 2014.

Published online September 24, 2014.

\section{INTRODUCTION}

Several physical phenomena have to be taken into account when modelling a water nuclear reactor such as $\mathrm{PWRs}^{6}$ or $\mathrm{BWRs}^{7}$ (see [12] for an introduction). In particular, the present work deals with the handling of high thermal dilation of the coolant fluid induced by thermal transfers in nuclear cores (see Fig. 1 for schematic pictures of PWR and BWR reactors). A natural approach is to represent the evolution of the flow by means of a system of PDEs similar to the compressible Navier-Stokes equations coupled to the modelling of phase transition as it is the case in classic industrial codes $[1,7,24]$.

In nominal and incidental situations as well as in some accidental situations studied in safety evaluations, the magnitude of the speed of sound is much higher than the one of the velocity of the coolant fluid, which means that the Mach number of the flow is small. The discretization of compressible Navier-Stokes type systems

Keywords and phrases. Low Mach number flows, modelling of phase transition, analytical solutions, method of characteristics, positivity-preserving schemes.

1 IFPEN - Lyon, BP 3, 69360 Solaize, France. manuel.bernard@ifpen.fr

2 DEN/DANS/DM2S/STMF, Commissariat à l'Énergie Atomique et aux Énergies Alternatives - Saclay, 91191 Gif-sur-Yvette, France. stephane.dellacherie@cea.fr

3 Université de Toulon - IMATH, EA 2134, avenue de l'Université, 83957 La Garde, France. faccanon@univ-tln.fr

4 MAP5 UMR CNRS 8145 - Université Paris Descartes, Sorbonne Paris Cité, 45 rue des Saints Pères, 75270 Paris Cedex 6, France. berenice.grec@parisdescartes.fr

5 CEREMA-INRIA - team ANGE and LJLL UMR CNRS 7598, 4 place Jussieu, 75005 Paris, France. yohan.penel@cerema.fr

${ }^{6} \mathrm{PWR}$ is the acronym for Pressurized Water Reactor.

${ }^{7} \mathrm{BWR}$ is the acronym for Boiling Water Reactor. 
at low Mach number may induce numerical issues directly related to the existence of fast acoustic waves (see for example $[15,28,42]$ when the convective part of the compressible Navier-Stokes system is discretized by means of a Godunov type scheme). Sereval numerical techniques based on the resolution of a Poisson equation have been proposed in the literature to extend incompressible methods to the (compressible) low Mach case. A pioneering work was that of Casulli and Greenspan [9] where a finite-difference scheme over a staggered grid is designed by impliciting terms involving the speed of sound in order to avoid restrictive stability conditions. In [11], Colella and Pao made use of the Hodge decomposition to single out the incompressible part of the velocity field.

Nevertheless, in a low Mach number regime, the acoustic phenomena can be neglected in energy balances although the flow is highly compressible because of the thermal dilation. Thus, to overcome the numerical difficulties, Dellacherie proposed in [16] another model obtained by filtering out the acoustic waves in the compressible model. Let us underline that this approach was first applied to model low Mach combustion phenomena [34,35,41], then astrophysical issues [4,5] and thermal dilation of the interface of bubbles at low Mach number [13]. This specific kind of models has been studied from a theoretical point of view. We mention for instance [21] for the well-posedness of a low Mach number system and $[26,34,39,41]$ for the derivation of monodimensional explicit solutions.

From a numerical point of view, 2D simulations have been performed in $[4,5,30]$ for astrophysical and combustion applications while a numerical study of the model established in [13] was proposed in [14]. As for the low Mach number model derived in [16] and called the Low Mach Nuclear Core (LMNC) model, it was discretized in [6] in the monodimensional (1D) case. Moreover, 1D unsteady analytical solutions were also given in $[6]$ which allowed to assess the numerical schemes. Notice that $2 \mathrm{D}$ numerical results will be presented in $[17,18]$.

Despite relevant numerical results, the approach proposed in $[6,16]$ was not satisfying since it was restricted to monophasic flows. Thus, we extend in this study the results stated in $[6,16]$ by taking into account phase transition in the LMNC model. If we neglect viscous effects, the LMNC model proposed in this paper may be seen as the low Mach number limit of the Homogeneous Equilibrium Model (HEM) [10,25,29,37,43] with source terms. Let us recall that the HEM model is the compressible Euler system in which the two phases are supposed to be at local kinematic, mechanic and thermodynamic equilibria.

A crucial step in the process is the modelling of fluid properties through the equation of state (EOS). It is important from a physical point of view to match experimental data and from a mathematical point of view to close the system of PDEs. In the present work, this point is achieved by using the stiffened gas EOS. A major result in this paper is the exhibition of 1D unsteady analytical solutions with phase transition (see Prop. 4.10). These solutions are of great importance: on the one hand they provide accurate estimates of heat transfers in a nuclear core in incidental and accidental situations, and on the other hand they enable to assess the robustness of the monodimensional numerical schemes presented in this article. In addition, regardless of the EOS used in the pure phases, when the thermodynamic pressure is constant (which is the case in the LMNC model) and when the phase change is modelled by assuming local mechanic and thermodynamic equilibria, the mixture can always be considered a stiffened gas (this point will be clarified in the sequel): this important remark legitimates the study of the LMNC model together with the stiffened gas EOS.

Compared to the numerous low Mach number combustion models derived in the literature, the LmNC model differs for several reasons. Some of them are due to the underlying fluids: indeed, combustion issues are related to gas modelled by the ideal gas law and involved in miscible mixtures whereas our modelling comprises a more general equation of state and mixtures of immiscible fluids. We must also mention that the system of PDEs is set in a bounded domain with nonperiodic boundary conditions whose influence upon theoretical and numerical investigations is noticeable.

At last, we wish to underline that although this study is specific to dimension 1 (which is essential to obtain in particular the unsteady analytical solutions with phase change), it remains useful from an industrial point of view since many safety evaluations use a 1D modelling to describe the flow in each component of the nuclear reactor and thus within the nuclear core [7,19]. Nevertheless, the extension of this work to dimensions 2 and 3 is a natural and important perspective [17].

This paper is organized as follows. In Section 2, the LMNC model is recalled together with boundary/initial conditions and assumptions under which it is valid. We also study the existence of (more or less) equivalent 


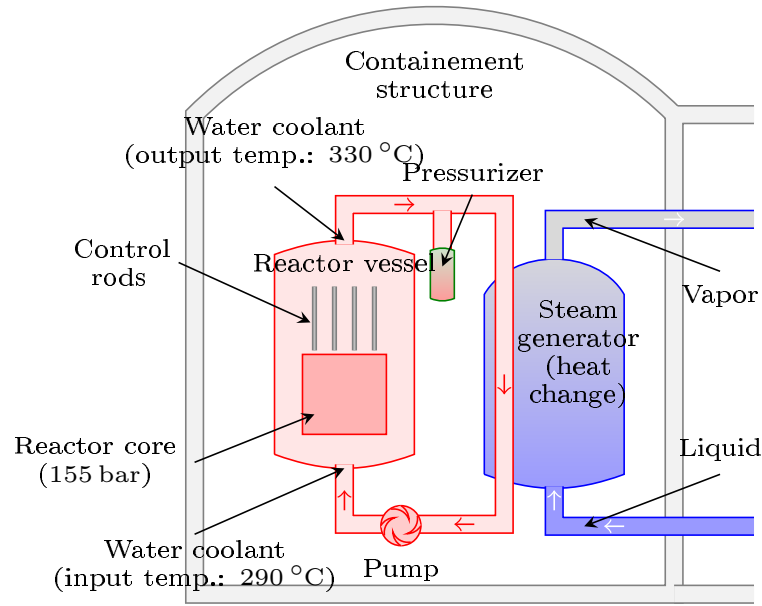

(A) Scheme of the primary circuit of a PWR.

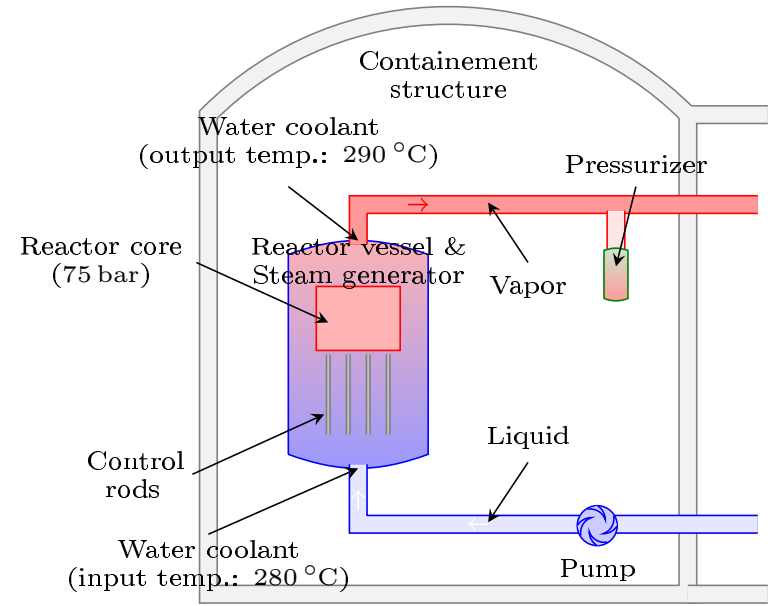

(в) Scheme of the primary circuit of a BWR.

FIGURE 1. Scheme of nuclear reactors whose coolant is water: the major difference between PWR and BWR is the steam void formation in the core of the latter.

formulations of the model that can be used depending on the variables we aim at focusing on. Section 3 is devoted to the modelling of phase transition and to the EOS that is necessary to close the system. In Section 4, we prove some theoretical results stated (without proof) in [6] and we extend them to the multiphasic case. Exact and asymptotic solutions are thus exhibited. Numerical aspects are then investigated in Section 5. More precisely, we present some numerical schemes based on the method of characteristics proposed in [38]. We then prove that these schemes preserve the positivity of the density and of the temperature for any time step. Finally, these schemes are applied in Section 6 to various situations with occurrence of phase transition like a simplified scenario for a Loss of Flow Accident (LOFA) induced by a coolant pump trip event.

\section{THE LOW MACH NUCLEAR CORE MODEL}

The Low Mach Nuclear Core (LMNC) model introduced in [16] is obtained by filtering out the acoustic waves in a compressible Navier-Stokes type system. This is achieved through an asymptotic expansion with respect to the Mach number assumed to be very small in this framework. One of the major consequences is the modification of the nature of the equations: the filtering out of the acoustic waves - which are solutions of a hyperbolic equation in the compressible system - introduces a new unknown (namely the dynamic pressure) which is solution to an elliptic equation in the LMNC model. Another consequence is that we are able to compute explicit monodimensional unsteady solutions of the LMNC model with or without phase transition ${ }^{8}$ (see Sect. 4) and to construct 1D robust and accurate numerical schemes ${ }^{9}$ (see Sect. 5).

In this section, we recall the LMNC model and we present equivalent formulations for smooth solutions. Since we are interested in the 1D case in this paper, we do not extend results to 2D/3D. Nevertheless, this can easily be done (provided the boundary conditions are adapted).

\subsection{Governing equations}

The 1D nonconservative formulation of the LMNC model [16] is written as

$$
\left\{\begin{array}{l}
\partial_{y} v=\frac{\beta\left(h, p_{0}\right)}{p_{0}} \Phi(t, y), \\
\rho\left(\partial_{t} h+v \partial_{y} h\right)=\Phi(t, y), \\
\partial_{t}(\rho v)+\partial_{y}\left(\rho v^{2}+\bar{p}\right)=\mathrm{F}(v)-\rho g,
\end{array} \quad(t, y) \in \mathbb{R}^{+} \times[0, L]\right.
$$

\footnotetext{
${ }^{8}$ This is not the case for the 1D compressible system from which the LMNC is derived.

${ }^{9}$ The existence of fast acoustic waves in the compressible system induces numerical difficulties - see [15,28] for example - which cannot arise in the LMNC model since the acoustic waves have been filtered out to obtain this low Mach number model.
} 
where $v$ and $h$ denote respectively the velocity and the (internal) enthalpy of the fluid. Pressure $p_{0}$ is a given constant - see below. The density $\rho=\rho\left(h, p_{0}\right)$ is related to the enthalpy by an equation of state (EOS) - see Section 3 . So does the dimensionless compressibility coefficient $\beta\left(h, p_{0}\right)$ which is defined by

$$
\beta\left(h, p_{0}\right) \stackrel{\text { def }}{=}-\frac{p_{0}}{\rho^{2}\left(h, p_{0}\right)} \cdot \frac{\partial \rho}{\partial h}\left(h, p_{0}\right) .
$$

The power density $\Phi(t, y)$ is a given function of time and space modelling the heating of the coolant fluid due to the fission reactions in the nuclear core. Finally, $g$ is the gravity field and $\mathrm{F}(v)$ models viscous effects: the classic internal friction in the fluid, and also the friction on the fluid due to technological devices in the nuclear core (e.g. the friction on the fluid due to the fuel rods). In the sequel, we take

$$
\mathrm{F}(v)=\partial_{y}\left(\mu \partial_{y} v\right) .
$$

In this case, $\mu$ is a turbulent viscosity given by an homogenized turbulent model. Nevertheless, we explain in the sequel that the exact choice of $\mathrm{F}(v)$ is not important in the $1 \mathrm{D}$ case (this is no more the case in $2 \mathrm{D} / 3 \mathrm{D}$ ).

We must also emphasize that model (2.1) is characterized by two pressure fields, which is classic in low Mach number approaches:

- $p_{0}$ : this field is involved in the equation of state and is named the thermodynamic pressure. It is assumed to be constant throughout the present study and corresponds to an average pressure in the nuclear core;

- $\bar{p}(t, y)$ : it appears in the momentum equation (2.1c) and is referred to as the dynamic pressure. It is similar to the pressure field which appears in the incompressible Navier-Stokes system.

Notice that $p_{0}+\bar{p}(t, y)$ is a 1st-order approximation of the classic compressible pressure in the nuclear core: the pressure $\bar{p}(t, y)$ is indeed a perturbation around the average pressure $p_{0}$ (this is due to the low Mach number hypothesis [16]). In the 1D case, equation (2.1c) decouples from the two other equations and may be considered a post-processing leading to the computation of $\bar{p}$ (this is why the expression of $\mathrm{F}(v)$ is not essential in 1D). Thus, equation (2.1c) will often be left apart in the sequel and equations (2.1a), (2.1b) will often be referred to as the LMNC model for the sake of simplicity.

\subsection{Supplements}

From now on, we suppose that:

\section{Hypothesis 2.1.}

(1) $\Phi(t, y)$ is nonnegative for all $(t, y) \in \mathbb{R}^{+} \times[0, L]$.

(2) $p_{0}$ is a positive constant.

The first assumption characterizes the fact that we study a nuclear core where the coolant fluid is heated. In the steam generator of a PWR type reactor (see Fig. 1a) - which could also be modelled by means of a LMNC type model - the fluid of the primary circuit heats the fluid of the secondary circuit by exchanging heat through a tube bundle: in that case, we would have $\Phi(t, y) \leq 0$ in the primary circuit and $\Phi(t, y) \geq 0$ in the secondary circuit.

The second assumption corresponds to real physical conditions even if it is not required in the setting of the equation of state (see Sect. 3.3).

Boundary conditions $(B C)$. The fluid is injected at the bottom of the core at a given enthalpy $h_{e}$ and at a given flow rate $D_{e}$. We also impose the dynamic pressure $\bar{p}$ at the exit of the core $(y=L)$. The inlet BC are

$$
\left\{\begin{array}{l}
h(t, 0)=h_{e}(t) \\
(\rho v)(t, 0)=D_{e}(t)
\end{array}\right.
$$

while the outlet $\mathrm{BC}$ is

$$
\bar{p}(t, L)=0 .
$$

The entrance velocity $v_{e}(t)$ to apply at $y=0$ is deduced from the relation

$$
v_{e}(t) \stackrel{\text { def }}{=} \frac{D_{e}(t)}{\rho_{e}(t)} \quad \text { where } \quad \rho_{e} \stackrel{\text { def }}{=} \rho\left(h_{e}, p=p_{0}\right) .
$$


The fact that $h_{e}$ and $D_{e}$ depend on time enables to model transient regimes induced by accidental situations. For example, when $D_{e}(t)$ tends to zero, it models a main coolant pump trip event which is a Loss Of Flow Accident (LOFA) as at the beginning of the Fukushima accident in the reactors 1, 2 and 3.

We also assume in the sequel that:

\section{Hypothesis 2.2.}

(1) $D_{e}$ is nonnegative.

(2) $h_{e}$ is such that $\rho_{e}$ is well-defined and positive.

The first assumption corresponds to a nuclear power plant of PWR or BWR type: the flow is upward ${ }^{10}$. The second assumption means that the $\operatorname{EOS} \rho(h, p)$ is such that $\rho\left(h_{e}, p_{0}\right)$ can be computed. Moreover, we also suppose that $\rho\left(h_{e}, p_{0}\right)>0$ from a physical point of view.

We finally make the following modelling hypothesis:

Hypothesis 2.3. $\beta$ is nonnegative.

Positivity assumptions about $\Phi, D_{e}, \beta$ and $\rho$ in Hypotheses 2.1, 2.2 and 2.3 ensure that the velocity $v(t, y)$ remains nonnegative at any time and anywhere in the core. Otherwise, the system could become ill-posed (see Sect. 4.2 in [16] where this question is partially addressed).

Well-prepared initial conditions. The model is finally closed by means of the initial condition $h_{0}(y)=h(0, y)$ satisfying the following hypothesis:

\section{Hypothesis 2.4.}

(1) We impose the compatibility condition $h_{0}(y=0)=h_{e}(t=0)$.

(2) $h_{0}$ is such that $\rho_{0}$ is well-defined and positive.

The initial density deduced from the EOS directly satisfies the equality $\rho_{0}(y=0)=\rho_{e}(t=0)$. Secondly, as system (2.1) consists of steady and unsteady equations, the initial velocity $v_{0}$ must satisfy equation (2.1a) for $t=0$, which means

$$
v_{0}^{\prime}(y)=\frac{\beta\left(h_{0}(y), p_{0}\right)}{p_{0}} \Phi(0, y) .
$$

Hence, $h_{0}$ prescribes the initial velocity $v_{0}$ through the previous differential equation together with the condition $v_{0}(y=0)=v_{e}(t=0)$. The initial flow rate $D_{0}$ is thus given by

$$
D_{0}(y)=\rho_{0}(y) v_{0}(y) \text {. }
$$

Such initial data $h_{0}$ and $D_{0}$ are said to be well-prepared. This will be implicitly assumed in the sequel.

\subsection{Origin and different formulations of the model}

The 1D LMNC model (2.1) is written in [16] as

$$
\left\{\begin{array}{l}
\partial_{y} v=\frac{\beta\left(h, p_{0}\right)}{p_{0}} \Phi(t, y), \\
\rho\left(h, p_{0}\right) \cdot\left(\partial_{t} h+v \partial_{y} h\right)=\Phi(t, y) .
\end{array}\right.
$$

We recall that in the $1 \mathrm{D}$ case, equation (2.1c) is a post-processing of (2.5). It is important to note that the low Mach number model (2.5) is justified only under smoothness assumptions. To study the existence of weak solutions, it might be better to use a conservative formulation which is equivalent to (2.5) for smooth solutions. This conservative formulation is the following:

Proposition 2.5. Under smoothness assumptions, system (2.5) is equivalent to

$$
\left\{\begin{array}{l}
\partial_{t} \rho+\partial_{y}(\rho v)=0 \\
\partial_{t}(\rho h)+\partial_{y}(\rho h v)=\Phi(t, y)
\end{array}\right.
$$

10 The flow could be downward when the nuclear reactor is a material testing reactor. 
System (2.6) (coupled to equation (2.1c)) is the LMNC model written in conservative variables. Although (2.6) is more general than (2.5), system (2.5) is interesting as it emphasizes the fact that the filtering out of the acoustic waves turns the hyperbolic nature of the compressible Navier-Stokes system (related to the acoustic waves) to an elliptic constraint (upon the velocity) similar to the incompressible case.

Moreover, under smoothness assumptions and for a particular class of EOS, we can derive a semi-conservative formulation equivalent to (2.5), and which may be useful to derive efficient numerical schemes. Indeed, we have the following proposition.

Proposition 2.6. Under smoothness assumptions:

(1) System (2.5) implies

$$
\left\{\begin{array}{l}
\partial_{y} v=\frac{\beta\left(h, p_{0}\right)}{p_{0}} \Phi(t, y) \\
\partial_{t}\left(\rho\left(h, p_{0}\right) h\right)+\partial_{y}\left(\rho\left(h, p_{0}\right) h v\right)=\Phi(t, y)
\end{array}\right.
$$

(2) For equations of state such that

$$
\frac{\partial \rho}{\partial h}\left(h, p_{0}\right) \neq-\frac{\rho\left(h, p_{0}\right)}{h},
$$

systems (2.5) and (2.7) are equivalent.

Condition (2.8) upon $\rho$ seems to be quite restrictive insofar as it does not enable to handle ideal gas (for which $\left.\frac{\partial \rho}{\partial h}=-\frac{\rho}{h}\right)$. In the latter case, equations $(2.7 \mathrm{a})$ and $(2.7 \mathrm{~b})$ are nothing but the same equation, which implies that we have to use formulations (2.5) or (2.6).

Remark 2.7. The equivalence between systems (2.5), (2.6) and (2.7) also holds in higher dimensions. Nevertheless, the momentum equation is strongly coupled to the other equations in $2 \mathrm{D} / 3 \mathrm{D}$ and must be taken into account under conservative or nonconservative forms. Indeed, these forms are equivalent (as soon as the unknowns are smooth).

Proof of Proposition 2.5.

According to definition (2.2) of $\beta$, we have

$$
\partial_{t} \rho+\partial_{y}(\rho v)=\frac{\partial \rho}{\partial h} \underbrace{\left(\partial_{t} h+v \partial_{y} h\right)}_{\stackrel{(2.5 \mathrm{~b})}{=} \frac{\Phi}{\rho}}+\rho \underbrace{\partial_{y} v}_{\stackrel{(2.5 \mathrm{a})}{=} \frac{\beta \Phi}{p_{0}}}=0
$$

which gives (2.6a). We also obtain

$$
\partial_{t}(\rho h)+\partial_{y}(\rho h v)=h\left(\partial_{t} \rho+\partial_{y}(\rho v)\right)+\rho\left(\partial_{t} h+v \partial_{y} h\right)=\Phi,
$$

using (2.5b) and (2.6a). We recover (2.6b).

$\Leftarrow$ Because of (2.6a), we deduce (2.5b) from (2.6b). Moreover, we deduce from (2.6a) and (2.5b) that

$$
\partial_{t} \rho+\partial_{y}(\rho v)=\frac{\partial \rho}{\partial h}\left(\partial_{t} h+v \partial_{y} h\right)+\rho \partial_{y} v=\frac{\partial \rho}{\partial h} \cdot \frac{\Phi}{\rho}+\rho \partial_{y} v=0,
$$

which gives (2.5a) thanks to definition $(2.2)$ of $\beta$.

Proof of Proposition 2.6. The first point is a direct consequence of Proposition 2.5 since

$$
\partial_{t}\left(\rho\left(h, p_{0}\right) h\right)+\partial_{y}\left(\rho\left(h, p_{0}\right) h v\right)=\underbrace{=\rho\left(h, p_{0}\right) \cdot\left(\partial_{t} h+v \partial_{y} h\right)}_{(2.5 \mathrm{~b})}+h \underbrace{\left[\partial_{t}\left(\rho\left(h, p_{0}\right)\right)+\partial_{y}\left(\rho\left(h, p_{0}\right) v\right)\right]}_{\stackrel{(2.9)}{=} 0} .
$$


To prove the second point, we just have to show that (2.7) implies (2.5) under condition (2.8). On the one hand, since

$$
\partial_{t} \rho+\partial_{y}(\rho v)=\frac{\partial \rho}{\partial h}\left(h, p_{0}\right)\left(\partial_{t} h+v \partial_{y} h\right)+\rho\left(h, p_{0}\right) \partial_{y} v,
$$

by using (2.2) and (2.7a), we obtain

$$
\partial_{t} \rho+\partial_{y}(\rho v)=\frac{\partial \rho}{\partial h}\left(h, p_{0}\right)\left(\partial_{t} h+v \partial_{y} h-\frac{\Phi}{\rho\left(h, p_{0}\right)}\right) .
$$

On the other hand, (2.7b) leads to

$$
\rho\left(h, p_{0}\right) \cdot\left(\partial_{t} h+v \partial_{y} h\right)+h\left[\partial_{t}\left(\rho\left(h, p_{0}\right)\right)+\partial_{y}\left(\rho\left(h, p_{0}\right) v\right)\right]=\Phi(t, y)
$$

that is to say

$$
\partial_{t} \rho+\partial_{y}(\rho v)=-\frac{\rho\left(h, p_{0}\right)}{h}\left(\partial_{t} h+v \partial_{y} h-\frac{\Phi}{\rho\left(h, p_{0}\right)}\right) .
$$

Thus, by comparing (2.10) and (2.11), we obtain

$$
\partial_{t} h+v \partial_{y} h-\frac{\Phi}{\rho\left(h, p_{0}\right)}=0
$$

under condition (2.8), which proves that (2.7) implies (2.5).

\section{Equation of State For tWo-Phase Fluids}

For the system to be closed, an additional equation is required: the equation of state (EOS). It corresponds to the modelling of thermodynamic properties and consists of an algebraic relation between thermodynamic variables. Indeed, perturbations of the inlet velocity or of the power density may strongly modify the temperature in the fluid and cause phase transition from liquid phase to vapor phase. At this modelling scale, the fluid can thus be under liquid, vapor or mixture phases. The issue is here to construct an EOS that models all phases of a fluid.

The model used in this study is based on the assumption of local mechanic and thermodynamic equilibria between phases. This means that the phases are assumed to move at the same velocity and that vaporisation, condensation and heat transfer processes are assumed to be instantaneous. As a consequence, the two-phase flow can be considered a single-phase problem provided the $\operatorname{EOS}(h, p) \mapsto \rho(h, p)$ (and thus the compressibility coefficient $\beta$ defined by (2.2)) takes phase transition into account. With this modelling, the two-phase flow evolution at low Mach number can be described by means of the LMNC model (2.1). In this case and when viscous effects modelled by $\mathrm{F}(v)$ in (2.1c) are neglected, the LMNC model (2.1) is the low Mach limit of the Homogeneous Equilibrium Model (HEM) [10, 25, 29, 37, 43] with source terms.

\subsection{General thermodynamics}

In classic thermodynamics, two variables are sufficient to represent a thermodynamic state of a pure singlephase fluid. This is done by means of an EOS which is a relation between the internal energy, the density and the entropy. In the literature, there exist numerous EOS specific to the fluid and to the model which are under consideration. In the case of liquid-vapor phase transitions, the EOS must not only represent the behavior of each pure phase (liquid or vapor), but also model the rate of the mass transfer between one phase to the other.

Phase diagram of Figure $2 \mathrm{~b}$ represent the coexistence curve $p^{s}(T)$ which relates the pressure to the temperature when phase change occurs: the plane $(T, p)$ is split by the coexistence curve into two regions in which one phase or another is stable. At any point on such a curve the two phases have equal Gibbs potentials and both phases can coexist.

In the $(1 / \rho, p)$ plane (see Fig. $2 \mathrm{~b}$ ) this mixture where both phases coexist is called the saturation zone. The designation "at saturation" means that the steam is in equilibrium with the liquid phase. This region is bounded by two curves connected at the critical point $\left(1 / \rho_{c}, p_{c}\right)$ which also belongs to the critical isotherm $T=T_{c}$. Within the two-phase region, through any point passes an isotherm which is a straight line. 


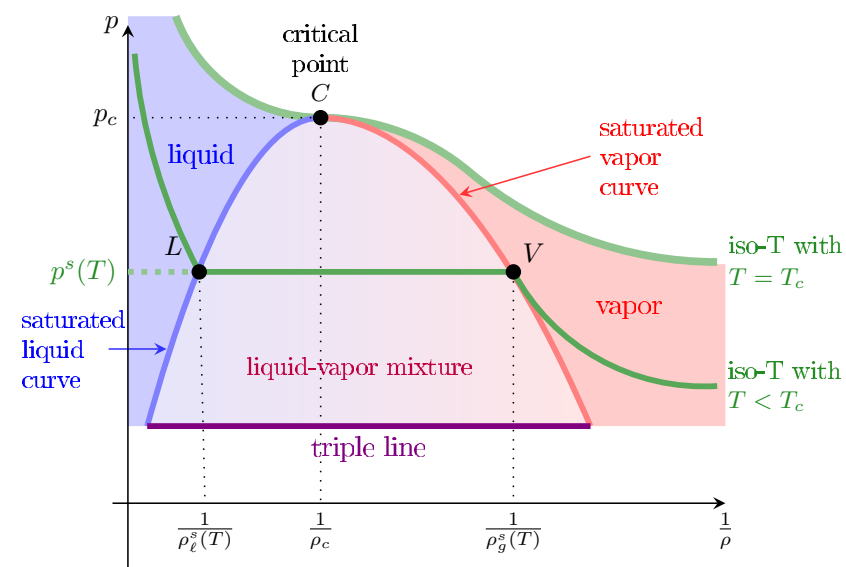

(A) Since the phase transition appears at constant pressure and temperature, the physical isotherm is horizontal in the mixture region and coincide with the isobar. At critical point $C$, the isotherm $T=T_{c}$ has a horizontal tangent.

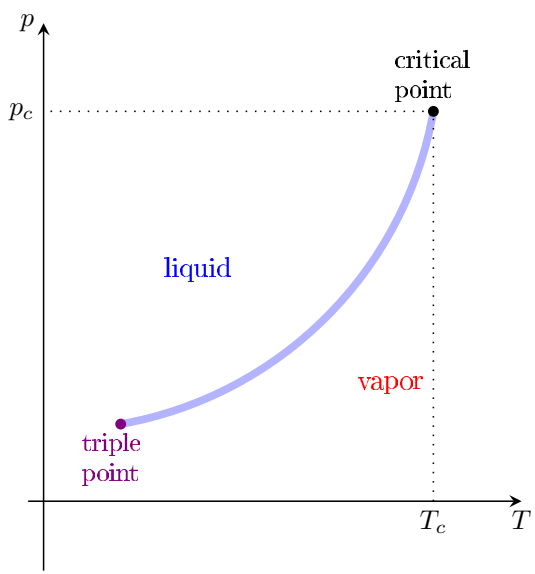

(в) Coexistence curve $p^{s}(T)$.

FigURE 2. Saturation and coexistence curves.

These curves can be obtained experimentally (see [33] for instance) and correspond to thermodynamic equilibria of temperature, pressure and Gibbs potential of the two phases. The Van der Waals law associated to the Maxwell construction is the most common example of this kind of EOS.

Nevertheless, it is very complicated to derive a unique EOS describing accurately both pure and mixture phases. To better handle pure phases and saturation curves, an idea consists in using two laws (one for each phase) so that each phase has its own thermodynamics. In the following section, we detail the general construction of the EOS in the mixture region given one EOS for each phase.

\subsection{Construction of the EOS in the mixture}

In this section, we explain how to specify the EOS in the mixture given an EOS for each pure phase.

Characterization of the two-phase media.

We consider each phase $\kappa(\kappa=\ell$ for the liquid phase and $\kappa=g$ for the vapor phase) as a compressible fluid governed by a given EOS: $(\rho, \varepsilon) \mapsto \eta_{\kappa}$ where $\rho, \varepsilon$ and $\eta_{\kappa}$ denote respectively the specific density, the specific internal energy and the specific entropy of the fluid. We assume that the function $(\tau, \varepsilon) \mapsto \eta_{\kappa}(1 / \tau, \varepsilon)$ has a negative-definite Hessian matrix where $\tau \stackrel{\text { def }}{=} 1 / \rho$ is the specific volume [8].

We then define classically for any phase $\kappa$ the temperature $T_{\kappa}$, the pressure $p_{\kappa}$ and the chemical potential $g_{\kappa}$ respectively by

$$
T_{\kappa} \stackrel{\text { def }}{=}\left(\left.\frac{\partial \eta_{\kappa}}{\partial \varepsilon}\right|_{\rho}\right)^{-1}, \quad p_{\kappa} \stackrel{\text { def }}{=}-\left.\rho^{2} T_{\kappa} \frac{\partial \eta_{\kappa}}{\partial \rho}\right|_{\varepsilon}, \quad g_{\kappa} \stackrel{\text { def }}{=} \varepsilon+\frac{p_{\kappa}}{\rho}-T_{\kappa} \eta_{\kappa} .
$$

Finally $\alpha$ denotes the volume fraction of vapor phase. This variable characterizes the volume of vapor in each unit volume: $\alpha=1$ means that this volume is completely filled by vapor; similarly, a full liquid volume corresponds to $\alpha=0$. Liquid and vapor are thus characterized by their thermodynamic properties.

The mixture density $\rho$ and the mixture internal energy $\varepsilon$ are defined by

$$
\left\{\begin{array}{l}
\rho \stackrel{\text { def }}{=} \alpha \rho_{g}+(1-\alpha) \rho_{\ell} \\
\rho \varepsilon \stackrel{\text { def }}{=} \alpha \rho_{g} \varepsilon_{g}+(1-\alpha) \rho_{\ell} \varepsilon_{\ell}
\end{array}\right.
$$


where $\rho_{g}, \rho_{\ell}, \varepsilon_{g}$ and $\varepsilon_{\ell}$ denote respectively vapor/liquid densities and vapor/liquid internal energies. Recalling that the internal energy is connected to the enthalpy by the relation $\rho h=\rho \varepsilon+p$, we can compute the mixture enthalpy $h$ when the pressure is the same in both phases (which is the case in the LMNC model where the thermodynamic pressure $p$ is constant and equal to $p_{0}$ ). This leads to

$$
\rho h=\alpha \rho_{g} h_{g}+(1-\alpha) \rho_{\ell} h_{\ell},
$$

where $h_{g}, h_{\ell}$ are respectively vapor/liquid enthalpies.

When taking phase transition into account, the two-phase mixture is constructed according to the second principle of thermodynamics. The key idea is that, when phases coexist (i.e. when $0<\alpha<1$ ), they have the same pressure, the same temperature and their chemical potentials are equal. The corresponding temperature, noted $T^{s}$ for temperature at saturation, is obtained by expliciting the equality of chemical potentials $g_{\ell}\left(p, T^{s}\right)=$ $g_{g}\left(p, T^{s}\right)$. This implies a relation between $T^{s}$ and $p$ (see for example [8,22,27] for more details). In the sequel we choose to express the temperature in the mixture as a function of the pressure and we define functions at saturation $\rho_{\kappa}^{s}$ and $h_{\kappa}^{s}$ by $p \mapsto \rho_{\kappa}^{s} \stackrel{\text { def }}{=} \rho_{\kappa}\left(p, T^{s}(p)\right)$ and $p \mapsto h_{\kappa}^{s} \stackrel{\text { def }}{=} h_{\kappa}\left(p, T^{s}(p)\right)$. Consequently, all thermodynamic quantities can be expressed as functions of the enthalpy and the pressure as it will be seen below. The choice to focus on pressure relies on the fact that the pressure in the LMNC model is supposed to be constant and equal to $p_{0}$.

Remark 3.1. Notice that for most fluids $h_{\ell}^{s}<h_{g}^{s}$ and $\rho_{\ell}^{s}>\rho_{g}^{s}$. In fact, the difference of enthalpies between the two phases in the saturated mixture is the latent heat of vaporisation

$$
L_{\ell g}^{s}(p) \stackrel{\text { def }}{=} h_{g}^{s}(p)-h_{\ell}^{s}(p)
$$

and we have the Clapeyron's law linking the latent heat of vaporisation to the slope of the coexistence curve (see $[8,27]$ for more details):

$$
L_{\ell g}^{s}(p)=\frac{\left(\frac{1}{\rho_{g}^{s}(p)}-\frac{1}{\rho_{\ell}^{s}(p)}\right) T^{s}(p)}{\left(T^{s}\right)^{\prime}(p)} .
$$

Density of the two-phase media.

Given functions at saturation, we are now able to model density in pure and mixture phases. Using equations (3.1a) and (3.2), the density is written as a function of enthalpy $h$ and pressure $p$ as follows

$$
\rho(h, p)= \begin{cases}\rho_{\ell}(h, p), & \text { if } h \leq h_{\ell}^{s}(p), \\ \rho_{m}(h, p)=\frac{\left[\rho_{g}^{s} \rho_{\ell}^{s}\left(h_{g}^{s}-h_{\ell}^{s}\right)\right](p)}{\left[\rho_{g}^{s} h_{g}^{s}-\rho_{\ell}^{s} h_{\ell}^{s}\right](p)-h \cdot\left[\rho_{g}^{s}-\rho_{\ell}^{s}\right](p)}, & \text { if } h_{\ell}^{s}(p)<h<h_{g}^{s}(p), \\ \rho_{g}(h, p), & \text { if } h \geq h_{g}^{s}(p) .\end{cases}
$$

For the derivation of this formula, see Appendix A. Notice that we have

$$
\alpha(h, p)= \begin{cases}0, & \text { if } h \leq h_{\ell}^{s}(p), \\ \frac{\rho_{\ell}^{s}(p)\left[h-h_{\ell}^{s}(p)\right]}{\left[\rho_{g}^{s} h_{g}^{s}-\rho_{\ell}^{s} h_{\ell}^{s}\right](p)-h \cdot\left[\rho_{g}^{s}-\rho_{\ell}^{s}\right](p)}, & \text { if } h_{\ell}^{s}(p)<h<h_{g}^{s}(p), \\ 1, & \text { if } h \geq h_{g}^{s}(p) .\end{cases}
$$

Temperature of the two-phase media. The temperature in the mixture $T^{s}$ is implicitly defined by the equation $g_{\ell}\left(p, T^{s}\right)=g_{g}\left(p, T^{s}\right)$ so that the temperature depends continuously on the enthalpy and on the pressure and reads

$$
T(h, p)= \begin{cases}T_{\ell}(h, p), & \text { if } h \leq h_{\ell}^{s}(p), \\ T^{s}(p), & \text { if } h_{\ell}^{s}(p)<h<h_{g}^{s}(p), \\ T_{g}(h, p), & \text { if } h \geq h_{g}^{s}(p) .\end{cases}
$$

We must emphasize that the function $h \mapsto T\left(h, p=p_{0}\right)$ cannot be inverted in the mixture zone for a constant pressure (as it is the case in the LMNC model). This remark prevents from working with equations on $T$ instead of equations on $h$. 
Compressibility coefficient of two-phase media. Computing the derivative of the density (3.3), we obtain the compressibility coefficient $\beta$ (previously defined by $(2.2)$ )

$$
\beta(h, p) \stackrel{\text { def }}{=}-\left.\frac{p}{\rho^{2}} \cdot \frac{\partial \rho}{\partial h}\right|_{p}= \begin{cases}\beta_{\ell}(h, p), & \text { if } h \leq h_{\ell}^{s}(p), \\ \beta_{m}(p) \stackrel{\text { def }}{=} p \cdot \frac{\frac{1}{\rho_{g}^{s}}-\frac{1}{\rho_{\ell}^{s}}}{h_{g}^{s}-h_{\ell}^{s}}(p), & \text { if } h_{\ell}^{s}(p)<h<h_{g}^{s}(p), \\ \beta_{g}(h, p), & \text { if } h \geq h_{g}^{s}(p) .\end{cases}
$$

We notice that independently of the EOS in the pure phases, the compressibility coefficient is constant in the mixture (since the pressure is constant in the LMNC model). Moreover, it is generally discontinuous between pure and mixture phases.

\subsection{The stiffened gas EOS}

Several EOS can be considered to describe thermodynamic properties of pure phases. In this paper (like in $[6,16])$ we use the stiffened gas law. This EOS is the simplest prototype that contains the main physical properties of pure fluids such as repulsive and attractive molecular effects, thereby facilitating the handling of thermodynamics through a simple analytical formulation. It is a generalization of the well-known ideal gas law (which is a commonly used EOS to describe the vapor phase), and it is an acceptable model for the liquid phase which is nearly incompressible (see Table 1 for water and steam parameters). Moreover, we will see that the EOS which models the mixture is a stiffened gas EOS regardless of the EOS modelling the pure phases.

For a given pure phase, the stiffened gas EOS is fully defined by the entropy $\eta$ written as a function of the density $\rho$ and the internal energy $\varepsilon$ :

$$
(\rho, \varepsilon) \mapsto \eta=c_{v}[\ln (\varepsilon-q-\pi / \rho)-(\gamma-1) \ln \rho]+m .
$$

The parameters $c_{v}>0$ (specific heat at constant volume), $\gamma>1$ (adiabatic coefficient), $\pi$ (constant reference pressure), $q$ (binding energy) and $m$ (reference entropy, relevant only when phase transition is involved) are some constants describing thermodynamic properties of the phase. Note that the case of an ideal gas is recovered by setting $\pi$ and $q$ to zero. We refer to [36] for a more in-depth discussion on the physical basis for this EOS.

The classic definitions in thermodynamics provide the following expressions for the temperature $T$, the pressure $p$, the enthalpy $h$ and the Gibbs potential $g$ as functions of the density $\rho$ and the internal energy $\varepsilon$ :

$$
\begin{aligned}
& p(\rho, \varepsilon) \stackrel{\text { def }}{=}-\left.T \rho^{2} \frac{\partial \eta}{\partial \rho}\right|_{\varepsilon}=(\gamma-1)(\varepsilon-q-\pi / \rho) \rho-\pi=(\gamma-1)(\varepsilon-q) \rho-\gamma \pi, \\
& T(\rho, \varepsilon) \stackrel{\text { def }}{=}\left(\left.\frac{\partial \eta}{\partial \varepsilon}\right|_{\rho}\right)^{-1}=\frac{\varepsilon-q-\pi / \rho}{c_{v}}, \\
& h(\rho, \varepsilon) \stackrel{\text { def }}{=} \varepsilon+\frac{p}{\rho}=q+(\varepsilon-q-\pi / \rho) \gamma, \\
& g(\rho, \varepsilon) \stackrel{\text { def }}{=} \varepsilon-T \eta+\frac{p}{\rho}=q+(\varepsilon-q-\pi / \rho)\left(\gamma-\frac{m}{c_{v}}-\ln \left((\varepsilon-q-\pi / \rho) \rho^{1-\gamma}\right)\right) .
\end{aligned}
$$

Physical considerations. We underline that the various parameters of the stiffened gas EOS cannot be chosen freely if a physically correct thermodynamic behavior is expected. Throughout this paper, we will consistently make the assumption that the parameters satisfy the following standard restrictions, which follow from thermodynamic stability theory.

For $\eta$ to be well-defined, it is necessary to have $\varepsilon-q-\pi / \rho>0$ and $\rho>0$. Since $c_{v}>0$, the first inequality implies $T>0$. Because of $\gamma>0$, we get $h-q>0$. Moreover, we also have to satisfy $\rho>0$ : since $\gamma>1$ and $h-q>0$, this is satisfied when $p+\pi>0$. We note that this hypothesis does not generally guarantee positivity of the pressure. This is consistent with the view that a stiffened gas is obtained by shifting the zero point of an ideal gas pressure [36]. In particular, all derived thermodynamic quantities are well defined as long as $p+\pi$ remains positive; see for instance [23]. Hence there is no reason to discard negative-pressure solutions as unphysical. To summarize, the modelling hypotheses upon the EOS are

$$
c_{v}>0, \quad \gamma>1 \quad \text { and } \quad p+\pi>0 .
$$


TABLE 1. Liquid water and steam, parameters computed in [31,32].

\begin{tabular}{lccccc}
\hline Phase & $c_{v}\left[\mathrm{~J} \cdot \mathrm{K}^{-1}\right]$ & $\gamma$ & $\pi[\mathrm{Pa}]$ & $q\left[\mathrm{~J} \cdot \mathrm{kg}^{-1}\right]$ & $q^{\prime}\left[\mathrm{J} \cdot \mathrm{K}^{-1}\right]$ \\
\hline Liquid & 1816.2 & 2.35 & $10^{9}$ & $-1167.056 \times 10^{3}$ & 0 \\
Vapor & 1040.14 & 1.43 & 0 & $2030.255 \times 10^{3}$ & -23310 \\
\hline
\end{tabular}

The term $(\gamma-1)(\varepsilon-q) \rho>0$ in the expression of $p$ models repulsive effects that are present for any state (gas, liquid or solid) and is due to molecular vibrations. $\pi$ leads to the "stiffened" properties compared to an ideal gas: a large value of $\pi$ implies near-incompressible behavior. The product $\gamma \pi>0$ represents the attractive molecular effect that guarantees the cohesion of matter in liquid or solid phases (hence $\pi=0$ for a gas).

Temperature and enthalpy at saturation. We assume that each phase $\kappa$ is described by its own stiffened gas EOS. To complete the results from Section 3.2, we have to express the temperature at saturation. As the temperature is constant in the mixture, we make a change of thermodynamic variables from $(\rho, \varepsilon)$ to $(p, T)$ which can be made explicit for this kind of EOS. The variables are now given by

$$
\begin{aligned}
\rho_{\kappa}(p, T) & =\frac{p+\pi_{\kappa}}{\left(\gamma_{\kappa}-1\right) c_{v_{\kappa}} T}, \\
\varepsilon_{\kappa}(p, T) & =c_{v_{\kappa}} T \frac{p+\pi_{\kappa} \gamma_{\kappa}}{p+\pi_{\kappa}}+q_{\kappa}, \\
h_{\kappa}(p, T) & =q_{\kappa}+\gamma_{\kappa} c_{v_{\kappa}} T, \\
g_{\kappa}(p, T) & =q_{\kappa}+T\left(c_{v_{\kappa}} \gamma_{\kappa}-q_{\kappa}^{\prime}-c_{v_{\kappa}} \gamma_{\kappa} \ln T+c_{v_{\kappa}}\left(\gamma_{\kappa}-1\right) \ln \left(p+\pi_{\kappa}\right)\right),
\end{aligned}
$$

where for the sake of simplicity we denoted

$$
q_{\kappa}^{\prime} \stackrel{\text { def }}{=} m_{\kappa}+c_{v_{\kappa}} \gamma_{\kappa} \ln c_{v_{\kappa}}+c_{v_{\kappa}}\left(\gamma_{\kappa}-1\right) \ln \left(\gamma_{\kappa}-1\right)
$$

as in $[31,40]$. We are now able to define the temperature at saturation $T^{s}(p)$ of the mixture as the solution of the equation $g_{\ell}\left(p, T^{s}\right)=g_{g}\left(p, T^{s}\right)$ which yields

$$
\left(c_{v_{g}} \gamma_{g}-c_{v_{\ell}} \gamma_{\ell}\right)\left[1-\ln T^{s}(p)\right]+c_{v_{g}}\left(\gamma_{g}-1\right) \ln \left(p+\pi_{g}\right)-c_{v_{\ell}}\left(\gamma_{\ell}-1\right) \ln \left(p+\pi_{\ell}\right)=\frac{q_{\ell}-q_{g}}{T^{s}(p)}+q_{g}^{\prime}-q_{\ell}^{\prime} .
$$

We suppose in the sequel that for $\kappa \in\{\ell, g\}, c_{v_{\kappa}}, \gamma_{\kappa}$ and $\pi_{\kappa}$ satisfy the modelling hypothesis (3.8), and that $q_{\kappa}$ and $q_{\kappa}^{\prime}$ are such that $T^{s}(p)$ exists and is unique at least when $p=p_{0}$ (this is the case for the constants of Table 1 computed for liquid and steam water). Thus, we have in particular $T^{s}\left(p_{0}\right)>0$. We remark that if $q_{\ell}=q_{g}$ or if $c_{v_{g}} \gamma_{g}=c_{v_{\ell}} \gamma_{\ell}$, we can compute $T^{s}$ analytically. Otherwise, a Newton algorithm can be used to solve this nonlinear equation for any fixed $p$. We then deduce the enthalpy at saturation for each phase

$$
h_{\kappa}^{s}(p)=q_{\kappa}+\gamma_{\kappa} c_{v_{\kappa}} T^{s}(p) .
$$

Density. The density is linked to the enthalpy by relation (3.3) where

$$
\begin{aligned}
\rho_{\kappa}(h, p) & =\frac{\gamma_{\kappa}}{\gamma_{\kappa}-1} \frac{p+\pi_{\kappa}}{h-q_{\kappa}}, \\
\rho_{\kappa}^{s}(p) & =\frac{p+\pi_{\kappa}}{\left(\gamma_{\kappa}-1\right) c_{v_{\kappa}} T^{s}(p)} .
\end{aligned}
$$

The density $\rho_{\kappa}^{s}(p)$ defines the density at saturation for each phase $\kappa \in\{\ell, g\}$.

Temperature. The temperature satisfies relation (3.5) with

$$
T_{\kappa}(h, p)=\frac{h-q_{\kappa}}{\gamma_{\kappa} c_{v_{\kappa}}}
$$


Compressibility coefficient. Relation (3.6) provides the expression of the compressibility coefficient with

$$
\beta(h, p)= \begin{cases}\beta_{\ell}(p)=\frac{\gamma_{\ell}-1}{\gamma_{\ell}} \frac{p}{p+\pi_{\ell}}, & \text { if } h \leq h_{\ell}^{s}(p), \\ \beta_{m}(p)=p \cdot \frac{\frac{1}{\rho_{g}^{s}}-\frac{1}{\rho_{\ell}^{s}}}{h_{g}^{s}-h_{\ell}^{s}}(p), & \text { if } h_{\ell}^{s}(p)<h<h_{g}^{s}(p), \\ \beta_{g}(p)=\frac{\gamma_{g}-1}{\gamma_{g}} \frac{p}{p+\pi_{g}}, & \text { if } h \geq h_{g}^{s}(p) .\end{cases}
$$

We notice that $\beta_{\kappa}$ is independent from $h$ whereas $\beta$ depends on $h$ through the choice of the phase $\kappa \in\{\ell, m, g\}$.

Binding energy. We set

$$
q(h, p) \stackrel{\text { def }}{=} \begin{cases}q_{\ell}, & \text { if } h \leq h_{\ell}^{s}(p), \\ q_{m}(p) \stackrel{\text { def }}{=}\left[\frac{\rho_{g}^{s} h_{g}^{s}-\rho_{\ell}^{s} h_{\ell}^{s}}{\rho_{g}^{s}-\rho_{\ell}^{s}}\right](p), & \text { if } h_{\ell}^{s}(p)<h<h_{g}^{s}(p), \\ q_{g}, & \text { if } h \geq h_{g}^{s}(p) .\end{cases}
$$

We notice that

$$
h_{\ell}^{s}(p)>q_{\ell}, \quad h_{g}^{s}(p)>q_{g} \quad \text { and } \quad h_{g}^{s}(p)>h_{\ell}^{s}(p)>q_{m}(p) .
$$

The two first estimates result from the definition (3.11) of $h_{\kappa}^{s}$ while the last one is proved by a straightforward calculation as $\left(h_{g}^{s}-h_{\ell}^{s}\right)\left(\rho_{g}^{s}-\rho_{\ell}^{s}\right)<0$ (see Fig. 3 and Rem. 3.1).

Graphs of density, temperature, compressibility coefficient and speed of sound (whose expression is detailed in Appendix B) for liquid water and steam at $p=155 \times 10^{5} \mathrm{~Pa}$ with parameters of Table 1 are pictured on Figure 3.

Conclusion: a unified stiffened gas EOS. Given relation (3.3), the density can be expressed by

$$
\rho(h, p)=\frac{p / \beta(h, p)}{h-q(h, p)}
$$

where $\beta(h, p)$ and $q(h, p)$ are given by (3.13) and (3.14). It is important to note that for any EOS defining the pure phases, $\rho(h, p)$ in the mixture is always given by $\rho_{m}(h, p)=\frac{p / \beta_{m}(p)}{h-q_{m}(p)}$. Thus, since $p$ is constant in the LMNC model, the mixture can always be considered a stiffened gas. Of course, this important property is mostly due to the local mechanic and thermodynamic equilibria hypothesis which gives $\rho_{m}(h, p)$ (see Appendix A).

Because of (3.16), PDE (2.1b) can be rewritten as

$$
\partial_{t} h+v \partial_{y} h=\frac{\beta\left(h, p_{0}\right)}{p_{0}}\left(h-q\left(h, p_{0}\right)\right) \Phi .
$$

This formulation is the key point of the present study and will be used in the sequel instead of (2.1b).

\section{TheOretical STUDY}

In this section we derive some analytical steady and unsteady solutions to system (2.1) together with BC (2.3) with stiffened gas law so that equation (2.1b) is replaced by (3.17). For a single phase flow we obtain exact and asymptotic solutions for different power densities and inlet velocities. We then extend these calculations to twophase flows with phase transition. We point out that our results generalize earlier works from Gonzalez-Santalo and Lahey [26]. In the latter paper, although the modelling of mixture is identical to what we present here, pure phases are considered incompressible. On the contrary, our work does not rely on any restriction in pure phases which allows for a physically more relevant modelling especially when pure gas phase appears. 


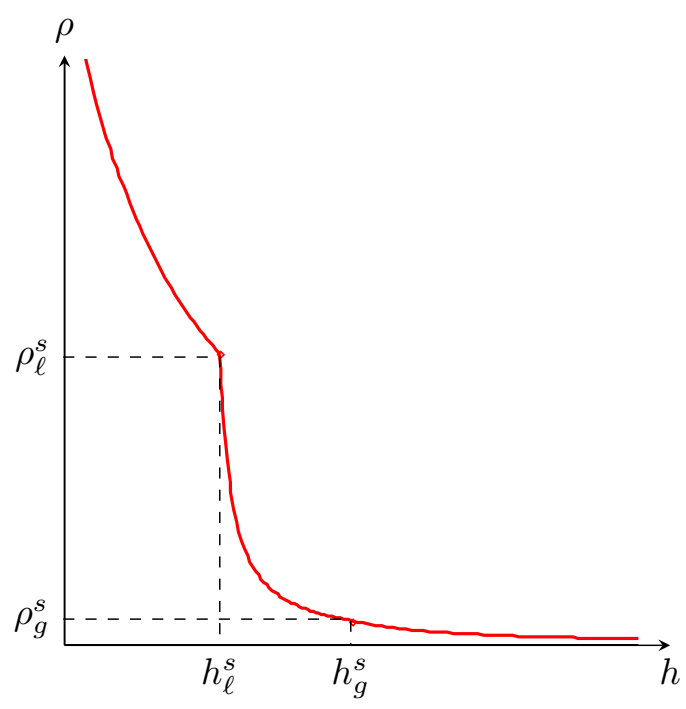

(A) Density as a function of the enthalpy: the densities of the liquid phase and the vapor phase at saturation are $\rho_{\ell}^{s} \approx 632.663 \mathrm{~kg} \cdot \mathrm{m}^{-3}$ and $\rho_{g}^{s} \approx$ $52.937 \mathrm{~kg} \cdot \mathrm{m}^{-3}$.

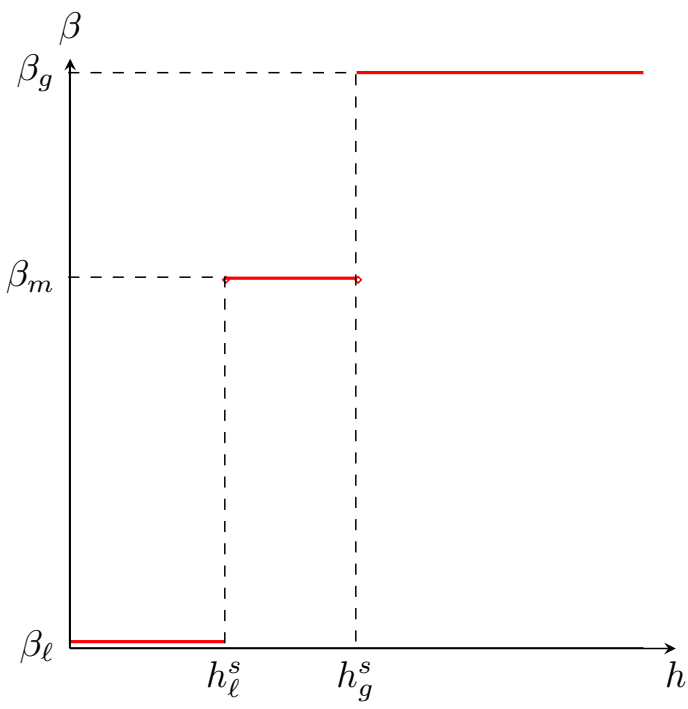

(c) Compressibility coefficient as a function of the enthalpy: the compressibility coefficients of the liquid phase, the mixture at saturation and the vapor phase are $\beta_{\ell} \approx 0.008768, \beta_{m} \approx 0.194852$ and $\beta_{g} \approx 0.300699$.

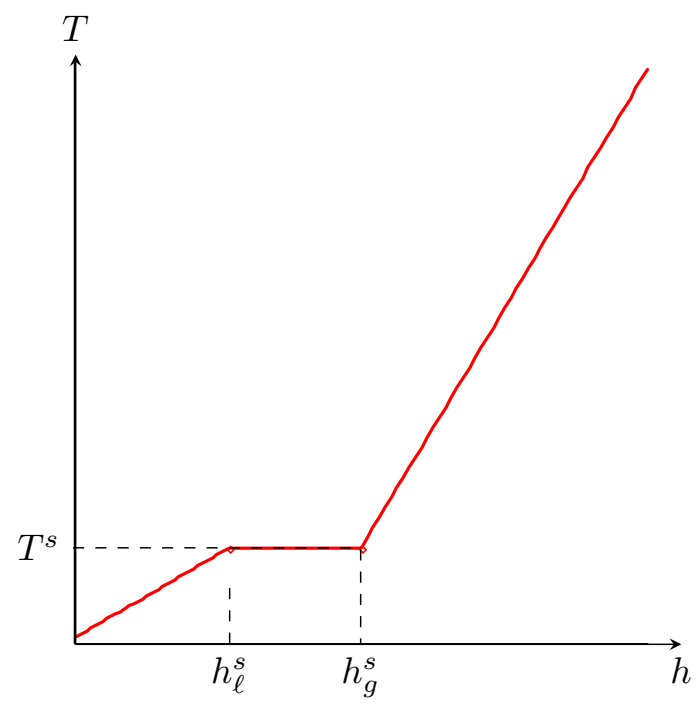

(в) Temperature as a function of the enthalpy: the temperature at saturation is $T^{s} \approx 654 \mathrm{~K}$.

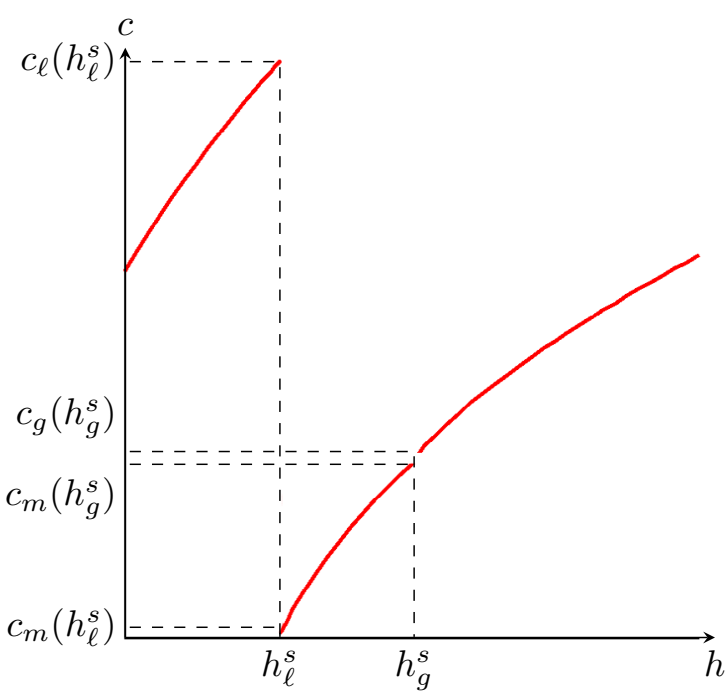

(D) Speed of sound as a function of the enthalpy: the speed of sound of the liquid phase and the vapor phase at saturation are $c_{\ell}\left(h_{\ell}^{s}\right) \approx 1942 \mathrm{~m} \cdot \mathrm{s}^{-1}$ and $c_{g}\left(h_{g}^{s}\right) \approx$ $647 \mathrm{~m} \cdot \mathrm{s}^{-1}$; in the mixture at saturation the speed of sound is in the range $\left(187 \mathrm{~m} \cdot \mathrm{s}^{-1}, 579 \mathrm{~m} \cdot \mathrm{s}^{-1}\right)$.

FiguRE 3. EOS with phase transition for parameters of Table 1 with $p_{0}=155 \times 10^{5} \mathrm{~Pa}$ : the enthalpies of the liquid and vapor phases at saturation are $h_{\ell}^{s} \approx 1.627 \times 10^{6} \mathrm{~J} \cdot \mathrm{K}^{-1}$ and $h_{g}^{s} \approx 3.004 \times 10^{6} \mathrm{~J} \cdot \mathrm{K}^{-1}$. 


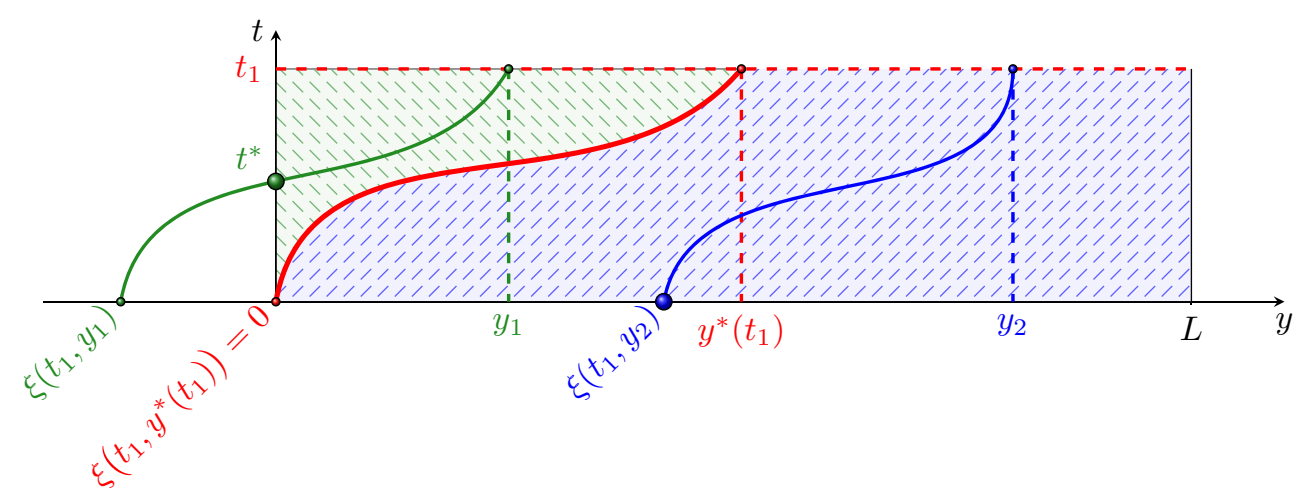

FIGURE 4. Sketch of the method of characteristics and definitions of $\xi(t, y), t^{*}(t, y)$ and $y^{*}(t)$.

\subsection{Validity of the model}

As stated in Section 3.3, we must ensure of the positivity of $h-q\left(h, p_{0}\right)$ for $h$ solution to (3.17). We mention that in the framework of stiffened gas satisfying (3.8), Hypotheses 2.2(2) and 2.4(2) reduce to:

Hypothesis 4.1. Data $h_{e}$ and $h_{0}$ are such that

$$
\mathcal{H} \stackrel{\text { def }}{=} \min \left\{\inf _{t \geq 0} h_{e}(t), \min _{y \in[0, L]} h_{0}(y)\right\}>q_{\ell} .
$$

Indeed, if at instant $t$ the inlet flow is in liquid phase (i.e. $\left.h_{e}(t)<h_{\ell}^{s}\right)$ and satisfies Hypothesis 4.1, then $h_{e}(t)-q\left(h_{e}(t), p_{0}\right)=h_{e}(t)-q_{\ell}>0$. If the inlet flow is a mixture of liquid and steam $\left(i . e . h_{e}(t) \in\left[h_{\ell}^{s}, h_{g}^{s}\right]\right)$, then $h_{e}(t)-q\left(h_{e}(t), p_{0}\right)=h_{e}(t)-q_{m} \geq h_{\ell}^{s}-q_{m}>0$ according to (3.15). Likewise, if the inlet flow is in vapor phase $\left(i . e . h_{e}(t)>h_{g}^{s}\right)$, then $h_{e}(t)-q\left(h_{e}(t), p_{0}\right)=h_{e}(t)-q_{g}>h_{g}^{s}-q_{g}>0$ according to (3.15). Thus, in each case, $h_{e}(t)-q\left(h_{e}(t), p_{0}\right)>0$ which implies that $\rho_{e}(t)$ is well-defined and positive through (3.8) and (3.12a). The same proof applies to $h_{0}$.

To go further, we need to define some notations that will be useful for the whole section. To solve the transport equation (3.17), we make use of the method of characteristics. This method consists in constructing curves (called characteristic curves) along which PDE (3.17) reduces to an ordinary differential equation (ODE). More precisely, let $\chi(\tau ; t, y)$ be the position at time $\tau$ of a particle located in $y$ at time $t$ in a flow driven at velocity $v$. For $t \geq 0$ and $y \in(0, L), \chi$ is thus solution to the parametrized ODE

$$
\left\{\begin{array}{l}
\frac{\mathrm{d} \chi}{\mathrm{d} \tau}(\tau ; t, y)=v(\tau, \chi(\tau ; t, y)), \\
\chi(t ; t, y)=y
\end{array}\right.
$$

The curve $(\tau, \chi(\tau ; t, y))$ is the characteristic curve passing through the point $(t, y)$. Properties of $\chi$ depend on the smoothness of the velocity field $v$.

We denote in the sequel (see Fig. 4) $\xi(t, y)$ the foot of the characteristic curve, $t^{*}(t, y)$ the time at which the characteristic curve crosses the boundary $y=0$ and $y^{*}(t)$ the location at time $t$ of a particle initially placed at $y=0$. In other words, $\xi, t^{*}$ and $y^{*}$ are defined by

$$
\xi(t, y) \stackrel{\text { def }}{=} \chi(0 ; t, y), \quad \chi\left(t^{*}(t, y) ; t, y\right)=0 \quad \text { and } \quad y^{*}(t) \stackrel{\text { def }}{=} \chi(t ; 0,0) .
$$

We thus have $\xi\left(t, y^{*}(t)\right)=0$ and also the characterization

$$
\xi(t, y)>0 \quad \Longleftrightarrow \quad t^{*}(t, y)<0 \quad \Longleftrightarrow \quad y>y^{*}(t) .
$$

This enables to prove the following result:

Lemma 4.2. When Hypothesis 4.1 is satisfied, any smooth solution to PDE (3.17) with BC (2.3a) and wellprepared initial conditions satisfies $h-q\left(h, p_{0}\right)>0$. 
Proof of Lemma 4.2. We recall that $q$ is defined through (3.14). For any $t \geq 0$ and $y \in(0, L)$, there are three possible situations:

- If $h(t, y)>h_{g}^{s}$, then $h(t, y)-q\left(h(t, y), p_{0}\right)>h_{g}^{s}-q_{g}>0$ because of (3.15);

- If $h(t, y) \in\left[h_{\ell}^{s}, h_{g}^{s}\right]$, then $h(t, y)-q\left(h(t, y), p_{0}\right) \geq h_{\ell}^{s}-q_{m}>0$ because of (3.15);

- If $h(t, y)<h_{\ell}^{s}$, then equation (3.17) reads (at least locally by a smoothness argument)

$$
\partial_{t}\left(h-q_{\ell}\right)+v \partial_{y}\left(h-q_{\ell}\right)=\frac{\beta_{\ell} \Phi}{p_{0}}\left(h-q_{\ell}\right) .
$$

We infer that $\hat{h}:(\tau ; t, y) \mapsto h(\tau, \chi(\tau ; t, y))$ satisfies

$$
\left\{\begin{array}{l}
\partial_{\tau}\left[\hat{h}(\tau ; t, y)-q_{\ell}\right]=\left[\hat{h}(\tau ; t, y)-q_{\ell}\right] \frac{\beta_{\ell}}{p_{0}} \Phi(\tau, \chi(\tau ; t, y)), \\
\hat{h}(t ; t, y)=h(t, y) .
\end{array}\right.
$$

Hence

$$
h(t, y)-q_{\ell}=\left[\hat{h}(\tau ; t, y)-q_{\ell}\right] \exp \left(\frac{\beta_{\ell}}{p_{0}} \int_{\tau}^{t} \Phi(\sigma, \chi(\sigma ; t, y)) \mathrm{d} \sigma\right)
$$

for any $\tau$ such that $\chi(\tau ; t, y) \in(0, L)$.

This shows that $h(t, y)-q_{\ell}$ is continuous and has the same sign

$\star$ as $h_{e}\left(t^{*}(t, y)\right)-q_{\ell}$ (if $\left.\xi(t, y) \leq 0\right)$,

$\star$ or as $h_{0}(\xi(t, y))-q_{\ell}$ (if $\left.\xi(t, y) \in[0, L]\right)$,

$\star$ or as $h_{\ell}^{s}-q_{\ell}$ (if $\xi(t, y)>L$ or if $\xi(t, y)$ does not exist, i.e. when phase change occurs before and after).

All of them are positive thanks to Hypothesis 4.1 and (3.15).

\subsection{Exact and asymptotic solutions for single-phase flow}

In this section, we compute some analytical solutions of (2.1) supplemented with the stiffened gas law for some particular cases (according to relevant values for $\Phi, h_{e}$ and $D_{e}$ ) when a single phase $\kappa \in\{\ell, m, g\}$ is present. The compressibility coefficient $\beta$ and the coefficient $q$ are thus constant.

Since we focus on the $1 \mathrm{D}$ case and as $\beta_{\kappa}\left(h, p_{0}\right)=\beta_{\kappa}$ in the case of the stiffened gas law, we can compute the velocity $v$ by a direct integration of equation (2.1a), which gives

$$
v(t, y)=v_{e}(t)+\frac{\beta_{\kappa}}{p_{0}} \int_{0}^{y} \Phi(t, z) \mathrm{d} z,
$$

with $v_{e}$ defined by (2.4). This velocity is obviously nonnegative under Hypotheses 2.1, 2.2 and 2.3, so that it is compatible with the location of BC. As mentioned earlier, equation (2.1b) can be rewritten as (3.17). To compute the enthalpy, we apply the method of characteristics (see above) which gets simpler in the case of the stiffened gas law.

The following results had first been stated in [6]. The proofs are detailed below.

\subsubsection{Constant power density}

Proposition 4.3. Let us assume that

- the power density $\Phi=\Phi_{0}>0$ is constant in time and space;

- $h_{e}$ and $D_{e}$ satisfy Hypotheses 2.2 and 4.1 and are such that $v_{e}=D_{e} / \rho\left(h_{e}, p_{0}\right)$ is independent of time;

- $h_{0}$ verifies Hypotheses 2.4 and 4.1 .

Let us denote $\hat{\Phi}_{0} \stackrel{\text { def }}{=} \beta_{\kappa} \Phi_{0} / p_{0}$. Then $\xi(t, y)$ and $t^{*}(t, y)$ defined by (4.2) are equal to

$$
\begin{aligned}
& \xi(t, y)=\left(y+\frac{v_{e}}{\hat{\Phi}_{0}}\right) \mathrm{e}^{-\hat{\Phi}_{0} t}-\frac{v_{e}}{\hat{\Phi}_{0}} \\
& t^{*}(t, y)=t-\frac{1}{\hat{\Phi}_{0}} \ln \left(1+\frac{\hat{\Phi}_{0}}{v_{e}} y\right)=-\frac{1}{\hat{\Phi}_{0}} \ln \left(1+\frac{\hat{\Phi}_{0}}{v_{e}} \xi(t, y)\right),
\end{aligned}
$$


and the solution $h$ of equation (2.1b) supplemented with $B C$ (2.3a) is given by

$$
h(t, y)= \begin{cases}q_{\kappa}+\left[h_{0}(\xi(t, y))-q_{\kappa}\right] \mathrm{e}^{\hat{\Phi}_{0} t}, & \text { if } \xi(t, y) \geq 0 \\ q_{\kappa}+\left[h_{e}\left(t^{*}(t, y)\right)-q_{\kappa}\right]\left(1+\frac{\hat{\Phi}_{0} y}{v_{e}}\right)=h_{e}\left(t^{*}(t, y)\right)+\frac{\Phi_{0} y}{D_{e}\left(t^{*}(t, y)\right)}, & \text { if } \xi(t, y)<0 .\end{cases}
$$

Corollary 4.4. Under the hypotheses of Proposition 4.3, we have $y^{*}(t)=\left(\mathrm{e}^{\hat{\Phi}_{0} t}-1\right) \frac{v_{e}}{\hat{\Phi}_{0}}$ and the solution $\bar{p}$ of equation (2.1c) together with $B C(2.3 \mathrm{c})$ is given by

- if $y^{*}(t)>L$, then

$$
\begin{aligned}
\bar{p}(t, y)=\hat{\Phi}_{0}[\mu(y)-\mu(L)]+ & \\
& \frac{v_{e} p_{0}}{\beta_{\kappa}}\left\{g\left[\bar{H}_{e}\left(t^{*}(t, y)\right)-\bar{H}_{e}\left(t^{*}(t, L)\right)\right]+\hat{\Phi}_{0} v_{e} \mathrm{e}^{\hat{\Phi}_{0} t}\left[\overline{\bar{H}}_{e}\left(t^{*}(t, y)\right)-\overline{\bar{H}}_{e}\left(t^{*}(t, L)\right)\right]\right\} ;
\end{aligned}
$$

- if $y^{*}(t) \leq L$ and $y \geq y^{*}(t)$ then

$$
\begin{aligned}
\bar{p}(t, y)= & \hat{\Phi}_{0}[\mu(y)-\mu(L)]+\frac{p_{0}}{\beta_{\kappa}}\left\{\left(g+\hat{\Phi}_{0} v_{e} \mathrm{e}^{\hat{\Phi}_{0} t}\right)\left[\bar{H}_{0}(\xi(t, L))-\bar{H}_{0}(\xi(t, y))\right]\right. \\
& \left.+\hat{\Phi}_{0}^{2} \mathrm{e}^{\hat{\Phi}_{0} t}\left[\overline{\bar{H}}_{0}(\xi(t, L))-\overline{\bar{H}}_{0}(\xi(t, y))\right]\right\}
\end{aligned}
$$

- otherwise

$$
\begin{aligned}
\bar{p}(t, y)= & \hat{\Phi}_{0}[\mu(y)-\mu(L)]+\frac{p_{0}}{\beta_{\kappa}}\left\{\left(g+\hat{\Phi}_{0} v_{e} \mathrm{e}^{\hat{\Phi}_{0} t}\right)\left[\bar{H}_{0}(\xi(t, L))-\bar{H}_{0}(0)\right]+\hat{\Phi}_{0}^{2} \mathrm{e}^{\hat{\Phi}_{0} t}\left[\overline{\bar{H}}_{0}(\xi(t, L))-\overline{\bar{H}}_{0}(0)\right]\right. \\
& \left.+v_{e}\left\{g\left[\bar{H}_{e}\left(t^{*}(t, y)\right)-\bar{H}_{e}(0)\right]+\hat{\Phi}_{0} v_{e} \mathrm{e}^{\hat{\Phi}_{0} t}\left[\overline{\bar{H}}_{e}\left(t^{*}(t, y)\right)-\overline{\bar{H}}_{e}(0)\right]\right\}\right\}
\end{aligned}
$$

where $\bar{H}_{0}^{\prime}(y)=1 /\left(h_{0}(y)-q_{\kappa}\right), \overline{\bar{H}}_{0}^{\prime}(y)=y /\left(h_{0}(y)-q_{\kappa}\right), \bar{H}_{e}^{\prime}(t)=1 /\left(h_{e}(t)-q_{\kappa}\right)$ and $\overline{\bar{H}}_{e}^{\prime}(t)=\mathrm{e}^{-\hat{\Phi}_{0} t} /\left(h_{e}(t)-q_{\kappa}\right)$.

Notice that equation $(4.4 \mathrm{c})$ is a correction of equation $(9 \mathrm{~b})$ in $[6]$.

Remark 4.5. As it has been stated in [6], if the inlet enthalpy is also constant and if the inlet velocity is nonzero, there is an asymptotic state which is reached in finite time. This time is equal to $t^{\infty}=\frac{1}{\hat{\Phi}_{0}} \ln \left(1+\frac{\hat{\Phi}_{0} L}{v_{e}}\right)$ and satisfies $\xi\left(t^{\infty}, L\right)=0$ and $y^{*}\left(t^{\infty}\right)=L$. Hence, for $t \geq t^{\infty}$, the solution $(h, v, p)(t, y)$ is given by

$$
\left\{\begin{array}{l}
h^{\infty}(y)=h_{e}+\frac{\Phi_{0}}{D_{e}} y, \\
v^{\infty}(y)=v_{e}+\hat{\Phi}_{0} y, \\
p^{\infty}(y)=\hat{\Phi}_{0}[\mu(y)-\mu(L)]+\frac{g D_{e}}{\hat{\Phi}_{0}} \ln \left(\frac{1+\frac{\hat{\Phi}_{0} L}{v_{e}}}{1+\frac{\hat{\Phi}_{0} y}{v_{e}}}\right)+\hat{\Phi}_{0} D_{e}(L-y) .
\end{array}\right.
$$

However, if the inlet velocity is $v_{e}=0$, then $\xi(t, y)$ is always positive and no asymptotic state can be reached since the enthalpy increases continuously in time.

Remark 4.6. Proposition 4.3 applies for nonzero $\Phi_{0}$. When $\Phi_{0}=0$, the same proof holds except the resolution of ODE (4.1) whose solution becomes $\chi(\tau ; t, y)=y+v_{e}(\tau-t)$. We remark that solution (4.6) converges to this case when $\hat{\Phi}_{0} \rightarrow 0$, which shows that the model is continuous with respect to $\Phi_{0}$. In particular, the enthalpy reads

$$
h(t, y)= \begin{cases}h_{0}\left(y-v_{e} t\right), & \text { if } y \geq v_{e} t \\ h_{e}\left(t-y / v_{e}\right), & \text { otherwise }\end{cases}
$$

which was expected as $h$ is a solution to a simple linear transport equation. 
Proof of Proposition 4.3. Equation (4.3) becomes

$$
v(t, y)=v_{e}+\hat{\Phi}_{0} y
$$

Since $v$ is linear, the Cauchy-Lipschitz theorem applied to ODE (4.1) ensures the existence of $\chi$ over some interval (depending on $t$ and $y$ ). Moreover, $\chi$ is continuous with respect to $(\tau, t, y)$. We then solve ODE (4.1) using expression (4.5). We obtain

$$
\chi(\tau ; t, y)=\left(y+\frac{v_{e}}{\hat{\Phi}_{0}}\right) \mathrm{e}^{\hat{\Phi}_{0}(\tau-t)}-\frac{v_{e}}{\hat{\Phi}_{0}} .
$$

For fixed $t \geq 0$ and $y \in(0, L)$, the requirement $\chi(\tau ; t, y) \in(0, L)$ constrains the interval of existence

$$
\tau \in\left(\max \left\{0 ; t^{*}(t, y)\right\}, t+\frac{1}{\hat{\Phi}_{0}} \ln \left(\frac{1+\frac{\hat{\Phi}_{0} L}{v_{e}}}{1+\frac{\hat{\phi}_{0} y}{v_{e}}}\right)\right) .
$$

For $(\tau, t, y)$ satisfying $(4.7)$, we note

$$
\hat{h}(\tau ; t, y) \stackrel{\text { def }}{=} h(\tau, \chi(\tau ; t, y)) .
$$

We deduce from equation (2.1b) rewritten under the form (3.17) that $\hat{h}$ satisfies

$$
\left\{\begin{array}{l}
\partial_{\tau}\left[\hat{h}(\tau ; t, y)-q_{\kappa}\right]=\hat{\Phi}_{0}\left[\hat{h}(\tau ; t, y)-q_{\kappa}\right] \\
\hat{h}(t ; t, y)=h(t, y)
\end{array}\right.
$$

The solution of this linear first order ODE is

$$
h(t, y)-q_{\kappa}=\hat{h}(t ; t, y)-q_{\kappa}=\left[\hat{h}(\tau ; t, y)-q_{\kappa}\right] \mathrm{e}^{\hat{\Phi}_{0}(t-\tau)}=\left[h(\tau, \chi(\tau ; t, y))-q_{\kappa}\right] \mathrm{e}^{{\hat{\phi_{0}}}_{0}(t-\tau)} .
$$

Two cases must be investigated depending on the minimal time until which the characteristic curve remains in the domain or equivalently depending on the sign of $\xi$ (see (4.7) and Fig. 4):

- if $\xi(t, y) \geq 0$, then the characteristic curve does not cross the boundary $y=0$, which means that we can take $\tau=0$ in equation (4.9) and

$$
h(t, y)-q_{\kappa}=\left[h_{0}(\chi(0 ; t, y))-q_{\kappa}\right] \mathrm{e}^{\hat{\phi}_{0} t}=\left[h_{0}(\xi(t, y))-q_{\kappa}\right] \mathrm{e}^{\hat{\Phi}_{0} t} ;
$$

- if $\xi(t, y)<0$, the backward characteristic curve reaches the boundary at time $t^{*}(t, y)>0$ and

$$
h(t, y)-q_{\kappa}=\left[h_{e}\left(t^{*}\right)-q_{\kappa}\right] \mathrm{e}^{\hat{\Phi}_{0}\left(t-t^{*}\right)}=\left[h_{e}\left(t^{*}\right)-q_{\kappa}\right]\left(1+\frac{\hat{\Phi}_{0} y}{v_{e}}\right) .
$$

Noticing that $\frac{\beta_{\kappa}}{p_{0}}\left(h-q_{\kappa}\right)=\frac{1}{\rho}$ leads to

$$
h(t, y)=q_{\kappa}+\left[h_{e}\left(t^{*}\right)-q_{\kappa}\right]+\frac{1}{\rho_{e}\left(t^{*}\right)} \frac{\Phi_{0}}{v_{e}} y=h_{e}\left(t^{*}\right)+\frac{\Phi_{0}}{D_{e}\left(t^{*}\right)} y .
$$

Proof of Corollary 4.4. The exact dynamic pressure $\bar{p}$ can be computed by integrating the momentum equation (2.1c) which is equivalent to

$$
\partial_{y} \bar{p}=-\partial_{t}(\rho v)-\partial_{y}\left(\rho v^{2}\right)+\partial_{y}\left(\mu \partial_{y} v\right)-\rho g .
$$

Using the mass conservation law and observing that $v$ given by (4.5) is independent of time, we obtain

$$
\partial_{y} \bar{p}=-\left(\hat{\Phi}_{0} v+g\right) \rho+\hat{\Phi}_{0} \partial_{y} \mu
$$


from which we deduce

$$
\partial_{y} \bar{p}=-\frac{p_{0}}{\beta_{\kappa}} \frac{g+\hat{\Phi}_{0}\left(v_{e}+\hat{\Phi}_{0} y\right)}{h-q_{\kappa}}+\hat{\Phi}_{0} \partial_{y} \mu .
$$

Integrating between $y$ and $L$, we get due to $\mathrm{BC}(2.3 \mathrm{c})$

$$
\bar{p}(t, y)=\frac{p_{0}\left(g+\hat{\Phi}_{0} v_{e}\right)}{\beta_{\kappa}} \int_{y}^{L} \frac{1}{\bar{h}(t, z)} \mathrm{d} z+\frac{p_{0} \hat{\Phi}_{0}^{2}}{\beta_{\kappa}} \int_{y}^{L} \frac{z}{\bar{h}(t, z)} \mathrm{d} z+\hat{\Phi}_{0}[\mu(y)-\mu(L)]
$$

where $\bar{h} \stackrel{\text { def }}{=} h-q_{\kappa}$. Since $h$ is defined piecewise by (4.4), we have to consider three cases:

- If $y^{*}(t)>L$, the curve $\tau \mapsto \chi(\tau ; t, y)$ lies in the green part of the graph on Figure 4, i.e. above the curve $\tau \mapsto \chi\left(\tau ; t, y^{*}(t)\right)$. As $y^{*}(t)>L \Longrightarrow \chi(t, y)<0$ for all $(t, y) \in \mathbb{R}_{+} \times(0, L)$, we can select the relevant value for $h$ in (4.4), i.e. $\bar{h}(t, y)=\bar{h}_{e}\left(t^{*}(t, y)\right)\left(1+\frac{\hat{\phi}_{0} y}{v_{e}}\right)$.

To compute each integral in (4.10), we use the change of variables $\tau=t^{*}(t, z)$, which yields

$$
\begin{aligned}
& \int_{y}^{L} \frac{1}{\bar{h}(t, z)} \mathrm{d} z=-v_{e} \int_{t^{*}(t, y)}^{t^{*}(t, L)} \frac{1}{\bar{h}_{e}(\tau)} \mathrm{d} \tau=v_{e}\left[\bar{H}_{e}(\tau)\right]_{t^{*}(t, L)}^{t^{*}(t, y)}, \\
& \int_{y}^{L} \frac{z}{\bar{h}(t, z)} \mathrm{d} z=\frac{v_{\mathrm{e}}^{2}}{\hat{\Phi}_{0}} \int_{t^{*}(t, L)}^{t^{*}(t, y)} \frac{\mathrm{e}^{\hat{\Phi}_{0}(t-\tau)}-1}{\bar{h}_{e}(\tau)} \mathrm{d} \tau=\frac{v_{\mathrm{e}}^{2}}{\hat{\Phi}_{0}}\left[\mathrm{e}^{\hat{\Phi}_{0} t} \overline{\bar{H}}_{e}(\tau)-\bar{H}_{e}(\tau)\right]_{t^{*}(t, L)}^{t^{*}(t, y)} .
\end{aligned}
$$

We then infer (4.4a).

- If $y^{*}(t) \leq L$ and $y \geq y^{*}(t)(\Longrightarrow \xi(t, y) \geq 0)$, we have $\bar{h}(t, y)=\bar{h}_{0}(\xi(t, y)) \mathrm{e}^{\hat{\Phi}_{0} t}$ so that by means of the change of variable $\zeta=\xi(t, z)$ we specify the integrals in (4.10)

$$
\begin{aligned}
\int_{y}^{L} \frac{1}{\bar{H}(t, z)} \mathrm{d} z & =\int_{\xi(t, y)}^{\xi(t, L)} \frac{1}{\bar{h}_{0}(\zeta)} \mathrm{d} \zeta=\left[\bar{H}_{0}(\zeta)\right]_{\xi(t, y)}^{\xi(t, L)}, \\
\int_{y}^{L} \frac{z}{\bar{h}(z)} \mathrm{d} z & =\mathrm{e}^{\hat{\Phi}_{0} t} \int_{\xi(t, y)}^{\xi(t, L)} \frac{\zeta}{\overline{h_{0}(\zeta)}} \mathrm{d} \zeta+\left(\mathrm{e}^{\hat{\Phi}_{0} t}-1\right) \frac{v_{e}}{\hat{\Phi}_{0}} \int_{\xi(t, y)}^{\xi(t, L)} \frac{1}{\overline{h_{0}(\zeta)}} \mathrm{d} \zeta \\
& =\mathrm{e}^{\hat{\Phi}_{0} t}\left[\overline{\bar{H}}_{0}(\zeta)\right]_{\xi(t, y)}^{\xi(t, L)}+\left(\mathrm{e}^{\hat{\Phi}_{0} t}-1\right) \frac{v_{e}}{\hat{\Phi}_{0}}\left[\bar{H}_{0}(\zeta)\right]_{\xi(t, y)}^{\xi(t, L)} .
\end{aligned}
$$

Hence we deduce (4.4b).

- The last corresponds to $y<y^{*}(t) \leq L$. The integration domain is split into two parts depending on the sign of $\xi(t, z)$. More precisely

$$
\int_{y}^{L} f(z) \mathrm{d} z=\int_{y^{*}(t)}^{L} f(z) \mathrm{d} z+\int_{y}^{y^{*}(t)} f(z) \mathrm{d} z
$$

for any function $f$. For integrals between $y^{*}(t)$ and $L$, we apply Formula (4.4b) with $y=y^{*}(t)$. For integrals between $y$ and $y^{*}(t)$, we apply Formula (4.4a) with $L$ replaced by $y^{*}(t)$. Summing all terms leads to (4.4c).

\subsubsection{Varying power density (with $y$ or $t$ )}

Let us generalize the results of Proposition 4.3 by taking $\Phi=\Phi(y)$ :

Proposition 4.7. Let us assume that

- the power density $\Phi$ depends only on space;

- $h_{e}$ and $D_{e}$ satisfy Hypotheses 2.2 and 4.1 and are such that $v_{e}=D_{e} / \rho\left(h_{e}, p_{0}\right)$ is independent of time;

- $h_{0}$ verifies Hypotheses 2.4 and 4.1 . 
Let us define

$$
\Theta(y) \stackrel{\text { def }}{=} \int_{0}^{y} \frac{\mathrm{d} z}{v(z)} \quad \text { with } \quad v(z)=v_{e}+\frac{\beta_{\kappa}}{p_{0}} \int_{0}^{z} \Phi(y) \mathrm{d} y .
$$

Then $\xi$ and $t^{*}$ defined by (4.2) are equal to

$$
\xi(t, y)=\Theta^{-1}(\Theta(y)-t) \quad \text { and } \quad t^{*}(t, y)=t-\Theta(y)
$$

The solution $h$ of equation (2.1b) with $B C$ (2.3a) is given by

$$
h(t, y)= \begin{cases}q_{\kappa}+v(y) \frac{h_{0}(\xi(t, y))-q_{\kappa}}{v(\xi(t, y))}, & \text { if } \xi(t, y) \geq 0 \\ q_{\kappa}+v(y) \frac{h_{e}\left(t^{*}(t, y)\right)-q_{\kappa}}{v_{e}}=h_{e}\left(t^{*}(t, y)\right)+\frac{1}{D_{e}\left(t^{*}(t, y)\right)} \int_{0}^{y} \Phi(z) \mathrm{d} z, & \text { if } \xi(t, y)<0\end{cases}
$$

Let us note that $v(y)=v_{0}(y)$ since $v(t, y)=v(y)$ and as $v_{0}$ is well-prepared (see Sect. 2.2).

We can also extend the results of Proposition 4.3 by taking $\Phi=\Phi(t)$ :

Proposition 4.8. Let us assume that

- the power density $\Phi$ depends only on time;

- $h_{e}$ and $D_{e}$ satisfy Hypotheses 2.2 and 4.1;

- $h_{0}$ verifies Hypotheses 2.4 and 4.1.

Let us define

$$
\Psi(t) \stackrel{\text { def }}{=} \frac{\beta_{\kappa}}{p_{0}} \int_{0}^{t} \Phi(s) \mathrm{d} s
$$

We thus have

$$
\xi(t, y)=y \mathrm{e}^{-\Psi(t)}-\int_{0}^{t} v_{e}(s) \mathrm{e}^{-\Psi(s)} \mathrm{d} s
$$

and $t^{*}(t, y)$ is the solution of the equation (upon $\left.t\right) y=\int_{t^{*}}^{t} v_{e}(s) \mathrm{e}^{\Psi(t)-\Psi(s)} d s$. Then the solution $h$ of equation (2.1b) with $B C(2.3 \mathrm{a})$ is given by

$$
h(t, y)=q_{\kappa}+ \begin{cases}{\left[h_{0}(\xi(t, y))-q_{\kappa}\right] \mathrm{e}^{\Psi(t)},} & \text { if } \xi(t, y) \geq 0 \\ {\left[h_{e}\left(t^{*}(t, y)\right)-q_{\kappa}\right] \mathrm{e}^{\Psi(t)-\Psi\left(t^{*}\right)},} & \text { if } \xi(t, y)<0\end{cases}
$$

Remark 4.9. In the general case, i.e. with time dependence for $h_{e}$ and $D_{e}$ and time/space dependence for $\Phi$, it does not seem possible to compute an exact solution. However, if $h_{e}(t), D_{e}(t)$ and $\Phi(t, y)$ have a finite limit as $t \rightarrow+\infty$ denoted by $h_{\mathrm{e}}^{\infty}, D_{\mathrm{e}}^{\infty}, \Phi^{\infty}(y)$, then there exists a steady solution for the enthalpy

$$
h^{\infty}(y)=h_{\mathrm{e}}^{\infty}+\frac{1}{D_{\mathrm{e}}^{\infty}} \int_{0}^{y} \Phi^{\infty}(z) \mathrm{d} z
$$

and all other quantities are deduced from $h^{\infty}$. We recover the result of [16].

Up to now, there is no theoretical result in the general case about the convergence of the unsteady solution to this steady state. We just notice that solutions from Propositions 4.7 and 4.8 actually do.

Proof of Proposition 4.7. Since the inlet velocity $v_{e}$ is a positive constant and as $\Phi$ does not depend on $t$, the velocity $v(t, y)=v(y)=v_{0}(y)$ is a positive function independent of time due to (4.3). Function $\Theta$ introduced in the statement of Proposition 4.7 is thus invertible. In a similar way as for Proposition 4.3, we solve the characteristic ODE (4.1) which is equivalent to

$$
1=\frac{\chi^{\prime}}{v(\chi)}=\Theta^{\prime}(\chi) \chi^{\prime}=[\Theta(\chi)]^{\prime}
$$


For the sake of simplicity, we set $\chi^{\prime}=\frac{\mathrm{d} \chi}{\mathrm{d} \tau}$ and $\chi^{\prime \prime}=\frac{\mathrm{d}^{2} \chi}{\mathrm{d} \tau^{2}}$. This leads to $\Theta(\chi(\tau ; t, y))-\Theta(y)=\Theta(\chi(\tau ; t, y))-$ $\Theta(\chi(t ; t, y))=\tau-t$ and

$$
\chi(\tau ; t, y)=\Theta^{-1}(\Theta(y)+\tau-t) .
$$

We can ensure that $\chi(\tau ; t, y) \in(0, L)$ provided $\tau \in\left(\max \left\{0, t^{*}(t, y)\right\}, t+\Theta(L)-\Theta(y)\right)$. Keeping the same notation (4.8) for $\hat{h}$, equation (3.17) becomes

$$
\left\{\begin{array}{l}
\partial_{\tau}\left[\hat{h}(\tau ; t, y)-q_{\kappa}\right]=\frac{\beta_{\kappa}}{p_{0}}\left[\hat{h}(\tau ; t, y)-q_{\kappa}\right] \Phi(\chi(\tau ; t, y)), \\
\hat{h}(t ; t, y)=h(t, y) .
\end{array}\right.
$$

We differentiate ODE (4.1) to obtain

$$
\chi^{\prime \prime}(\tau ; t, y)=\chi^{\prime}(\tau ; t, y) \frac{\mathrm{d} v}{\mathrm{~d} y}(\chi(\tau ; t, y))=\chi^{\prime}(\tau ; t, y) \frac{\beta_{\kappa}}{p_{0}} \Phi(\chi(\tau ; t, y)) .
$$

The positivity of $v$ implies that $\chi^{\prime}(\tau ; t, y)>0$ and

$$
\frac{\beta_{\kappa}}{p_{0}} \Phi(\chi(\tau ; t, y))=\frac{\chi^{\prime \prime}(\tau ; t, y)}{\chi^{\prime}(\tau ; t, y)}=\left[\ln \left(\chi^{\prime}(\tau ; t, y)\right)\right]^{\prime}=[\ln v(\chi(\tau ; t, y))]^{\prime} .
$$

Inserting this relation in equation (4.12), we have

$$
\partial_{\tau}\left(\hat{h}-q_{\kappa}\right)=\left(\hat{h}-q_{\kappa}\right) \partial_{\tau}[\ln v(\chi(\tau ; t, y))] .
$$

Hence

$$
h(t, y)-q_{\kappa}=v(y) \frac{h(\tau, \chi(\tau ; t, y))-q_{\kappa}}{v(\chi(\tau ; t, y))} .
$$

Given expression (4.11) for $\chi$, we finally obtain

$$
h(t, y)=q_{\kappa}+ \begin{cases}v(y) \frac{h_{0}(\xi(t, y))-q_{\kappa}}{v(\xi(t, y))}, & \text { if } \xi(t, y) \geq 0, \\ v(y) \frac{h_{e}\left(t^{*}(t, y)\right)-q_{\kappa}}{v_{e}}, & \text { otherwise. }\end{cases}
$$

Proof of Proposition 4.8. As previously, the key point is the integration of the characteristic ODE (4.1) which reads

$$
\frac{\mathrm{d}}{\mathrm{d} \tau}\left(\chi(\tau) \mathrm{e}^{-\Psi(\tau)}\right)=v_{e}(\tau) \mathrm{e}^{-\Psi(\tau)}
$$

This leads to

$$
\chi(\tau ; t, y)=y \mathrm{e}^{\Psi(\tau)-\Psi(t)}+\int_{t}^{\tau} v_{e}(s) \mathrm{e}^{\Psi(\tau)-\Psi(s)} \mathrm{d} s .
$$

Consequently

$$
h(t, y)-q_{\kappa}=\left[h(\tau, \chi(\tau ; t, y))-q_{\kappa}\right] \mathrm{e}^{\Psi(t)-\Psi(\tau)} .
$$

The main issue is then to determine the interval for $\tau$ such that $\chi(\tau ; t, y) \in(0, L)$. The equality $\chi(\tau ; t, y)=0$ can be rewritten as

$$
y=\int_{\tau}^{t} v_{e}(s) \mathrm{e}^{\Psi(t)-\Psi(s)} \mathrm{d} s .
$$

As the right hand side vanishes for $\tau=t$, two cases may occur: either $y$ is greater than the right hand side for $\tau=0$ (which would imply that the left bound of the interval is 0 ), or there exists $\tau=t^{*}>0$ such that the previous equality holds. In the former case, we obtain

$$
h(t, y)-q_{\kappa}=\left[h_{0}(\chi(0 ; t, y))-q_{\kappa}\right] \mathrm{e}^{\Psi(t)},
$$

while in the latter case

$$
h(t, y)-q_{\kappa}=\left[h_{e}\left(t^{*}(t, y)\right)-q_{\kappa}\right] \mathrm{e}^{\Psi(t)-\Psi\left(t^{*}\right)} .
$$




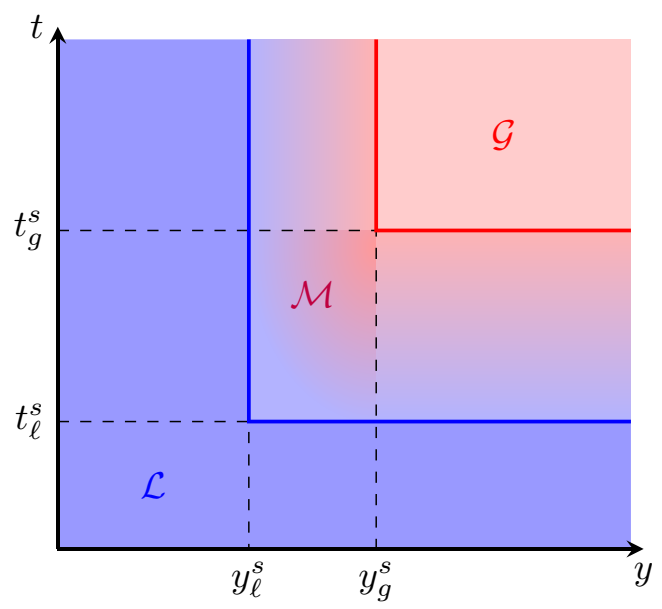

(A) Liquid $(\mathcal{L})$, mixture $(\mathcal{M})$ and vapor $(\mathcal{G})$ regions of the spatiotemporal domain $\mathbb{R}^{+} \times$ $\mathbb{R}^{+}$for the velocity.

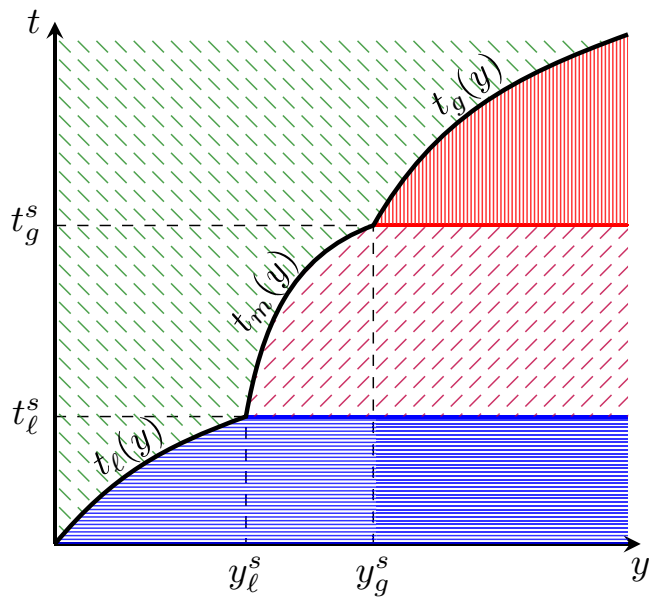

(B) Definition of four regions of the spatiotemporal domain $\mathbb{R}^{+} \times \mathbb{R}^{+}$for the enthalpy.

Figure 5. Definition of regions for Proposition 4.10.

\subsection{Exact and asymptotic solutions for two-phase flow with phase transition}

In the case of a two-phase flow with phase transition and if we can determine the position of each phase, we can deduce the exact solution using the single-phase flow results given in Section 4.2. In the particular case where all parameters and boundary/initial data are constant, the result is the following.

Proposition 4.10. Let us assume that $h_{e}(t) \equiv h_{e}>q_{\ell}, v_{e}(t) \equiv v_{e}>0, \Phi(t, y) \equiv \Phi_{0}$ and $h_{0}(y) \equiv h_{0}=h_{e}>q_{\ell}$. Let $\hat{\Phi}_{\kappa} \stackrel{\text { def }}{=} \beta_{\kappa} \Phi_{0} / p_{0}$, where $\kappa$ is respectively $\ell, m, g$ in the liquid, mixture and gas phases. We suppose that the initial and boundary data correspond to the liquid phase, i.e. $h_{e}\left(=h_{0}\right)<h_{\ell}^{s}$. We set

$$
y_{\ell}^{s} \stackrel{\text { def }}{=} \frac{D_{e}}{\Phi_{0}}\left(h_{\ell}^{s}-h_{e}\right), \quad y_{g}^{s} \stackrel{\text { def }}{=} \frac{D_{e}}{\Phi_{0}}\left(h_{g}^{s}-h_{e}\right) .
$$

Let us define three curves in $\mathbb{R}^{+} \times \mathbb{R}^{+}$as pictured on Figure $5 B$

$$
\begin{array}{ll}
t_{\ell}(y) \stackrel{\text { def }}{=} \frac{1}{\hat{\Phi}_{\ell}} \ln \left(1+\frac{\hat{\Phi}_{\ell} y}{v_{e}}\right), & \text { for } 0 \leq y \leq y_{\ell}^{s}, \\
t_{m}(y) \stackrel{\text { def }}{=} \frac{1}{\hat{\Phi}_{m}} \ln \left(\frac{v_{e}+\left(\hat{\Phi}_{\ell}-\hat{\Phi}_{m}\right) y_{\ell}^{s}+\hat{\Phi}_{m} y}{v_{e}+\hat{\Phi}_{\ell} y_{\ell}^{s}}\right)+t_{\ell}\left(y_{\ell}^{s}\right), & \text { for } y_{\ell}^{s}<y<y_{g}^{s}, \\
t_{g}(y) \stackrel{\text { def }}{=} \frac{1}{\hat{\Phi}_{g}} \ln \left(\frac{v_{e}+\left(\hat{\Phi}_{\ell}-\hat{\Phi}_{m}\right) y_{\ell}^{s}+\left(\hat{\Phi}_{m}-\hat{\Phi}_{g}\right) y_{g}^{s}+\hat{\Phi}_{g} y}{v_{e}+\left(\hat{\Phi}_{\ell}-\hat{\Phi}_{m}\right) y_{\ell}^{s}+\hat{\Phi}_{m} y_{g}^{s}}\right)+t_{m}\left(y_{g}^{s}\right), & \text { for } y \geq y_{g}^{s} .
\end{array}
$$

Let us also define

$$
t_{\ell}^{s} \stackrel{\text { def }}{=} t_{\ell}\left(y_{\ell}^{s}\right)=\frac{1}{\hat{\Phi}_{\ell}} \ln \left(\frac{h_{\ell}^{s}-q_{\ell}}{h_{0}-q_{\ell}}\right), \quad t_{g}^{s} \stackrel{\text { def }}{=} t_{m}\left(y_{g}^{s}\right)=t_{\ell}^{s}+\frac{1}{\hat{\Phi}_{m}} \ln \left(\frac{h_{g}^{s}-q_{m}}{h_{\ell}^{s}-q_{m}}\right) .
$$

Then the spatiotemporal domain $\mathbb{R}^{+} \times \mathbb{R}^{+}$consists of three regions corresponding to liquid, mixture and vapor phases as follows (see Fig. 5A):

$$
\begin{aligned}
\mathcal{L} & =\left\{(t, y) \in \mathbb{R}^{+} \times \mathbb{R}^{+} \mid t \leq t_{\ell}^{s} \text { or } y \leq y_{\ell}^{s}\right\}, \\
\mathcal{M} & =\left\{(t, y) \in\left(t_{\ell}^{s},+\infty\right) \times\left(y_{\ell}^{s},+\infty\right) \mid t \leq t_{g}^{s} \text { or } y \leq y_{g}^{s}\right\}, \\
\mathcal{G} & =\left\{(t, y) \in\left(t_{g}^{s},+\infty\right) \times\left(y_{g}^{s},+\infty\right)\right\} ;
\end{aligned}
$$


the solution $v$ of equation (2.1a) is given by

$$
v(t, y)= \begin{cases}v_{e}+\hat{\Phi}_{\ell} y, & \text { if }(t, y) \in \mathcal{L}, \\ v_{e}+\hat{\Phi}_{\ell} y_{\ell}^{s}+\hat{\Phi}_{m}\left(y-y_{\ell}^{s}\right), & \text { if }(t, y) \in \mathcal{M}, \\ v_{e}+\hat{\Phi}_{\ell} y_{\ell}^{s}+\hat{\Phi}_{m}\left(y_{g}^{s}-y_{\ell}^{s}\right)+\hat{\Phi}_{g}\left(y-y_{g}^{s}\right), & \text { if }(t, y) \in \mathcal{G},\end{cases}
$$

and the solution $h$ of equation (2.1b) is given by (see Fig. 5B)

$$
h(t, y)= \begin{cases}q_{\ell}+\left(h_{0}-q_{\ell}\right) \mathrm{e}^{\hat{\Phi}_{\ell} t}, & \text { if }(t, y) \in \mathcal{L} \text { and } t<t_{\ell}(y), \\ q_{m}+\left(h_{\ell}^{s}-q_{m}\right) \mathrm{e}^{\hat{\Phi}_{m}\left(t-t_{\ell}^{s}\right)}, & \text { if }(t, y) \in \mathcal{M} \text { and } t<t_{m}(y), \\ q_{g}+\left(h_{g}^{s}-q_{g}\right) \mathrm{e}^{\hat{\Phi}_{g}\left(t-t_{g}^{s}\right)}, & \text { if }(t, y) \in \mathcal{G} \text { and } t<t_{g}(y), \\ h_{e}+\frac{\Phi_{0}}{D_{e}} y, & \text { otherwise. }\end{cases}
$$

Remark 4.11. We notice that constant $\hat{\Phi}_{m}$ is the Zuber's characteristic reaction frequency defined in [44] and used in [26]. Contrary to the latter paper, we do not assume the pure phases to be incompressible. Thus we can extend the Zuber's frequency to liquid and gas phases as a piecewise constant function.

Remark 4.12. In any case, we observe that a steady state is reached. It is given by

$$
h^{\infty}(y)=h_{e}+\frac{\Phi_{0}}{D_{e}} y
$$

and the velocity is piecewise linear. Moreover, we can determine the time $t^{\infty}$ at which the steady state (it is thus an asymptotic state in that case) is reached

$$
t^{\infty} \stackrel{\text { def }}{=} \begin{cases}t_{\ell}(L), & \text { if } \quad y_{\ell}^{s}>L \\ t_{m}(L), & \text { if } \quad y_{\ell}^{s} \leq L \leq y_{g}^{s} \\ t_{g}(L), & \text { if } \quad y_{g}^{s}<L\end{cases}
$$

The assumptions of Proposition 4.10 may seem restrictive but it can be trivially extended to several cases:

- to other constant initial and boundary conditions (i.e. mixture or vapor);

- if $\Phi=\Phi_{0}<0$ such that $v$ remains positive, the enthalpy is then still monotone.

If $\Phi$ and/or boundary-initial conditions are not constant anymore, the enthalpy is no longer monotone. It is thus difficult to determine regions $\mathcal{L}, \mathcal{M}$ and $\mathcal{G}$. However, if this can be achieved, we can similarly apply the monophasic results in each region to compute the exact solution. In any case, the following result holds (similar to Remark 4.9):

Remark 4.13. For any inlet velocity $v_{e}$, inlet flow rate $D_{e}$ and power density $\Phi$ which have finite limits in time, let us denote $\left(h_{\mathrm{e}}^{\infty}, D_{\mathrm{e}}^{\infty}, \Phi^{\infty}(y)\right)=\lim _{t \rightarrow+\infty}\left(h_{e}(t), D_{e}(t), \Phi(t, y)\right)$. Then, there exists a steady solution $\left(h^{\infty}(y), v^{\infty}(y), \bar{p}^{\infty}(y)\right)$ given by

$$
\begin{aligned}
h^{\infty}(y) & =h_{\mathrm{e}}^{\infty}+\frac{1}{D_{\mathrm{e}}^{\infty}} \int_{0}^{y} \Phi^{\infty}(z) \mathrm{d} z, \\
v^{\infty}(y) & =\frac{D_{\mathrm{e}}^{\infty}}{\rho\left(h^{\infty}(y), p_{0}\right)}, \\
\bar{p}^{\infty}(y) & =g \int_{y}^{L} \rho\left(h^{\infty}(z), p_{0}\right) \mathrm{d} z-\left[\mu \frac{\beta\left(h^{\infty}(z), p_{0}\right) \Phi^{\infty}(z)}{p_{0}}-\frac{\left(D_{\mathrm{e}}^{\infty}\right)^{2}}{\rho\left(h^{\infty}(z), p_{0}\right)}\right]_{z=y}^{z=L},
\end{aligned}
$$

with $\rho\left(h^{\infty}, p_{0}\right)$ given by $(3.3)$. 
Proof of Proposition 4.10. Since $\rho_{e}, v_{e}, h_{0}$ and $\Phi_{0}$ are constant and correspond to the liquid phase, equations (2.1a), (3.17) are written over a time interval $[0, \mathcal{T}$ ) for some $\mathcal{T}>0$ to be specified later (which corresponds to the first time another phase appears)

$$
\left\{\begin{array}{l}
\partial_{y} v=\hat{\Phi}_{\ell}, \\
\partial_{t} h+v \partial_{y} h=\hat{\Phi}_{\ell}\left(h-q_{\ell}\right), \\
v(t, 0)=v_{e}, \\
h(t, 0)=h_{e}, \\
h(0, x)=h_{0}=h_{e} .
\end{array}\right.
$$

We can apply Proposition 4.3 to this system which leads to

$$
v(y)=v_{e}+\hat{\Phi}_{\ell} y
$$

and

$$
h(t, y)= \begin{cases}q_{\ell}+\left(h_{0}-q_{\ell}\right) \mathrm{e}^{\hat{\Phi}_{\ell} t}, & \text { if } t<t_{\ell}(y), \\ h_{e}+\frac{\Phi_{0}}{D_{e}} y, & \text { otherwise }\end{cases}
$$

where the curve $t=t_{\ell}(y)$ corresponds to the characteristic curve coming from $(t=0, y=0)$, that is $\xi_{\ell}(t, y)=$ 0 (see Prop. 4.3 for the definition of $\xi_{\ell}$ ). The enthalpy $h(t, \cdot)$ is a monotone-increasing function consisting (spatially) of a linear part and a constant part at each time. Two situations may occur:

- either $h_{e}+\frac{\Phi_{0}}{D_{e}} L \leq h_{\ell}^{s}$ : the fluid remains liquid indefinitely $(\mathcal{T}=+\infty)$ and the enthalpy is equal to $h_{e}+\frac{\Phi_{0}}{D_{e}} y$ everywhere as soon as $t \geq t_{\ell}(L)$;

- or $h_{e}+\frac{\Phi_{0}}{D_{e}} L>h_{\ell}^{s}$ : a mixture phase appears.

In the latter case, there exists $t>0$ and $y \in(0, L)$ such that $h(t, y)=h_{\ell}^{s}$. We then define $\mathcal{T}=t_{\ell}^{s}$ as the solution of $h\left(t_{\ell}^{s}, L\right)=h_{\ell}^{s}$ and $y_{\ell}^{s}$ as the smallest $y$ such that $h\left(t_{\ell}^{s}, y\right)=h_{\ell}^{s}$, i.e.

$$
q_{\ell}+\left(h_{0}-q_{\ell}\right) \mathrm{e}^{\hat{\Phi}_{\ell} t_{\ell}^{s}}=h_{\ell}^{s}, \quad h_{e}+\frac{\Phi_{0}}{D_{e}} y_{\ell}^{s}=h_{\ell}^{s} .
$$

For $t>t_{\ell}^{s}$, the fluid is in liquid phase for $y<y_{\ell}^{s}$ and in mixture phase for $y \geq y_{\ell}^{s}$ and $t$ small enough, i.e. $t \in\left[t_{\ell}^{s}, \mathcal{T}^{\prime}\right)$. Therefore, in the liquid region the previous solution is still valid, whereas in the mixture region $\left[t_{\ell}^{s}, \mathcal{T}^{\prime}\right) \times\left[y_{\ell}^{s}, L\right]$ equations $(2.1 \mathrm{a}),(3.17)$ are written as

$$
\left\{\begin{array}{l}
\partial_{y} v=\hat{\Phi}_{m} \\
\partial_{t} h+v \partial_{y} h=\hat{\Phi}_{m}\left(h-q_{m}\right) \\
v\left(t, y_{\ell}^{s}\right)=v_{e}+\hat{\Phi}_{\ell} y_{\ell}^{s} \\
h\left(t, y_{\ell}^{s}\right)=h_{\ell}^{s} \\
h\left(t_{\ell}^{s}, y\right)=h_{\ell}^{s}
\end{array}\right.
$$

We adapt Proposition 4.3 to the current spatiotemporal domain so that we obtain

$$
v(y)=v_{e}+\hat{\Phi}_{\ell} y_{\ell}^{s}+\hat{\Phi}_{m}\left(y-y_{\ell}^{s}\right)
$$

and

$$
h(t, y)= \begin{cases}q_{m}+\left(h_{\ell}^{s}-q_{m}\right) \mathrm{e}^{\hat{\Phi}_{m}\left(t-t_{\ell}^{s}\right)}, & \text { if } t<t_{m}(y), \\ h_{e}+\frac{\Phi}{D_{e}} y, & \text { otherwise. }\end{cases}
$$

The curve $t=t_{m}(y)$ corresponds to the characteristic curve passing through $\left(t_{\ell}^{s}, y_{\ell}^{s}\right)$, i.e.

$$
t_{m}(y)=\frac{1}{\hat{\Phi}_{m}} \ln \left(\frac{v_{e}+\left(\hat{\Phi}_{\ell}-\hat{\Phi}_{m}\right) y_{\ell}^{s}+\hat{\Phi}_{m} y}{v_{e}+\hat{\Phi}_{\ell} y_{\ell}^{s}}\right)+t_{\ell}^{s} .
$$




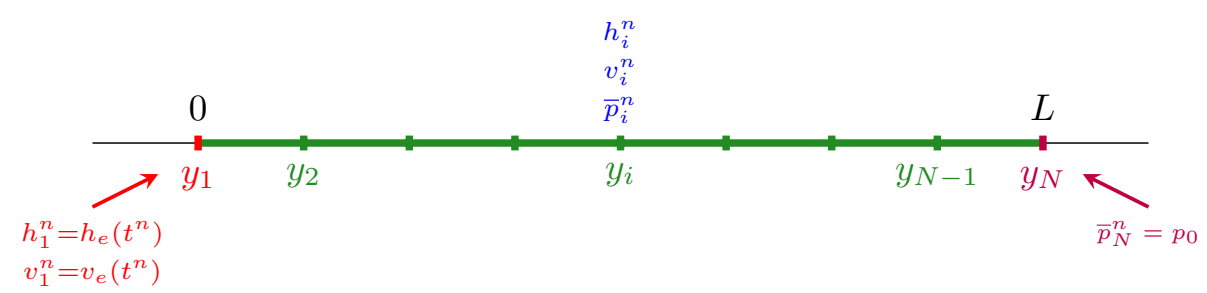

FiguRE 6. Grid and boundary conditions.

This can be rewritten in a simpler form. To this end, we use the definition of $y_{\ell}^{s}$, the relation $\frac{1}{\rho_{e}}=\frac{\beta_{\ell}}{p_{0}}\left(h_{e}-q_{\ell}\right)$ and the continuity of $\rho$ at $y_{\ell}^{s}$ (implying $\beta_{m}\left(h_{\ell}^{s}-q_{m}\right)=\beta_{\ell}\left(h_{\ell}^{s}-q_{\ell}\right)$ ) which leads to

$$
t_{m}(y)=\frac{1}{\hat{\Phi}_{m}} \ln \left(\frac{h_{e}-q_{m}+\frac{\Phi_{0} y}{D_{e}}}{h_{\ell}^{s}-q_{m}}\right)+t_{\ell}^{s} .
$$

If needed, we apply the same procedure to find $\left(t_{g}^{s}, y_{g}^{s}\right)$ such that $h_{g}^{s}$ is reached and the proof follows.

\section{NUMERICAL SCHEME}

The main advantage of dimension 1 is that equations in (2.1) can be decoupled in an explicit numerical procedure which means that it suffices to compute $h$ from equation $(2.1 \mathrm{~b})$ - i.e. (3.17) for the stiffened gas law - in order to deduce all other variables. To solve the transport equation with source term (3.17), the numerical method of characteristics (MOC) seems suitable insofar as this method was essential in the theoretical part of the study. Moreover, this method has interesting properties such as unconditional stability and positivitypreserving for variable $h-q$ (see Sect. 5.3). Accuracy can be improved using 2nd-order results from [38].

Given $\Delta y>0$ and $\Delta t>0$, we consider a uniform Cartesian grid $\left\{y_{i}=i \Delta y\right\}_{1<i<N}$ such that $y_{1}=0$ and $y_{N}=L$ (see Fig. 6) as well as a time discretization $\left\{t^{n}=n \Delta t\right\}_{n \geq 0}$. Unknowns are collocated at the nodes of the mesh. We set the initial values $v_{i}^{0}=v_{0}\left(y_{i}\right)$ and $h_{i}^{0}=h_{0}\left(y_{i}\right)$ for $i=1, \ldots, N$.

We must emphasize that as the pressure variable $p$ is supposed to be constant and equal to $p_{0}$ in the LMNC model, most parameters are constant throughout the study (such as $T^{s}, \beta_{\kappa}$ or $q_{\kappa}$ ). That is why references to the dependence on the pressure may be dropped in the sequel for the sake of simplicity.

\subsection{Key idea of the scheme}

For details about numerical methods of characteristics, the reader may refer to $[2,20,38]$ and references therein. This method amounts to tracking particles along the flow and to solve ODEs along the characteristic curves. More precisely, it consists in locating accurately the position of particles and updating the unknown according to the source term as described from a theoretical point of view in Section 4.2. Actually, at time $t^{n}$, we aim at approximating the solution of

$$
\frac{\mathrm{d}}{\mathrm{d} \tau} \hat{h}_{i}^{n+1}(\tau)=\frac{\beta\left(\hat{h}_{i}^{n+1}(\tau)\right) \Phi\left(\tau, \chi\left(\tau ; t^{n+1}, y_{i}\right)\right)}{p_{0}}\left(\hat{h}_{i}^{n+1}(\tau)-q\left(\hat{h}_{i}^{n+1}(\tau)\right)\right)
$$

where $\tau \mapsto \hat{h}_{i}^{n+1}(\tau) \stackrel{\text { def }}{=} h\left(\tau, \chi\left(\tau ; t^{n+1}, y_{i}\right)\right)$ and the characteristic flow $\chi$ satisfies

$$
\left\{\begin{array}{l}
\frac{\mathrm{d}}{\mathrm{d} \tau} \chi\left(\tau ; t^{n+1}, y_{i}\right)=v\left(\tau, \chi\left(\tau ; t^{n+1}, y_{i}\right)\right), \quad \tau \leq t^{n+1}, \\
\chi\left(t^{n+1} ; t^{n+1}, y_{i}\right)=y_{i}
\end{array}\right.
$$

with $v$ an approximate solution to (2.1a). The reader may refer to [38] for further details about the resolution of $(5.1 \mathrm{~b})$. 
The classic Euler-type MOC method applied to ODE (5.1a) over the interval $\left[t^{n} ; t^{n+1}\right]$ is written

$$
\begin{aligned}
h\left(t^{n+1}, y_{i}\right) & =\hat{h}_{i}^{n+1}\left(t^{n+1}\right) \\
& =\hat{h}_{i}^{n+1}\left(t^{n}\right)+\Delta t \frac{\beta\left(\hat{h}_{i}^{n+1}\left(t^{n}\right)\right) \Phi\left(t^{n}, \chi\left(t^{n} ; t^{n+1}, y_{i}\right)\right)}{p_{0}}\left(\hat{h}_{i}^{n+1}\left(t^{n}\right)-q\left(\hat{h}_{i}^{n+1}\left(t^{n}\right)\right)\right)+\mathcal{O}\left(\Delta t^{2}\right) .
\end{aligned}
$$

Hence the scheme can be written

$$
h_{i}^{n+1}=\tilde{h}_{i}^{n+1, n}+\Delta t \frac{\beta\left(\tilde{h}_{i}^{n+1, n}\right) \Phi\left(t^{n}, \xi_{i}^{n}\right)}{p_{0}}\left(\tilde{h}_{i}^{n+1, n}-q\left(\tilde{h}_{i}^{n+1, n}\right)\right),
$$

where $\xi_{i}^{n}$ and $\tilde{h}_{i}^{n+1, n}$ are some approximations of $\chi\left(t^{n} ; t^{n+1}, y_{i}\right)$ and $\hat{h}_{i}^{n+1}\left(t^{n}\right)$, respectively. In the sequel, scheme (5.2) will be referred to as the MOC scheme.

To reach higher order, we rewrite ODE (5.1a) using the facts that $\beta(h)$ and $h-q(h)$ are positive under Hypotheses 2.3 and 4.1. We set

$$
R(\hat{h}) \stackrel{\text { def }}{=} \int_{\mathcal{H}}^{\hat{h}} \frac{\mathrm{d} h}{\beta(h) \cdot(h-q(h))},
$$

where $\mathcal{H}$ is defined within Hypothesis 4.1. Then, equation (5.1a) reads

$$
R^{\prime}\left(\hat{h}_{i}^{n+1}\right) \frac{\mathrm{d}}{\mathrm{d} \tau} \hat{h}_{i}^{n+1}(\tau)=\frac{\Phi\left(\tau, \chi\left(\tau ; t^{n+1}, y_{i}\right)\right)}{p_{0}}
$$

and thus can be integrated explicitly between $t^{n}$ and $t^{n+1}$

$$
R\left(\hat{h}_{i}^{n+1}\left(t^{n+1}\right)\right)-R\left(\hat{h}_{i}^{n+1}\left(t^{n}\right)\right)=\frac{1}{p_{0}} \int_{t^{n}}^{t^{n+1}} \Phi\left(\tau, \chi\left(\tau ; t^{n+1}, y_{i}\right)\right) \mathrm{d} \tau .
$$

As $\Phi$ is a datum, the right hand side can be expanded at any order or exactly computed. Using the trapezoidal rule, the scheme reads

$$
h_{i}^{n+1}=R^{-1}\left(R\left(\tilde{h}_{i}^{n+1, n}\right)+\frac{\Delta t}{p_{0}} \frac{\Phi\left(t^{n}, \xi_{i}^{n}\right)+\Phi\left(t^{n+1}, y_{i}\right)}{2}\right)
$$

Notice that we can give explicit expressions for $R$ and $R^{-1}$ :

$$
\begin{gathered}
R(h)= \begin{cases}\frac{1}{\beta_{\ell}} \ln \left(\frac{h-q_{\ell}}{\mathcal{H}-q_{\ell}}\right), & \text { if } h \leq h_{\ell}^{s}, \\
R_{\ell}^{s}+\frac{1}{\beta_{m}} \ln \left(\frac{h-q_{m}}{h_{\ell}^{s}-q_{m}}\right), & \text { if } h_{\ell}^{s}<h<h_{g}^{s}, \\
R_{g}^{s}+\frac{1}{\beta_{g}} \ln \left(\frac{h-q_{g}}{h_{g}^{s}-q_{g}}\right), & \text { if } h \geq h_{g}^{s},\end{cases} \\
R^{-1}(r)= \begin{cases}q_{\ell}+\left(\mathcal{H}-q_{\ell}\right) \mathrm{e}^{\beta_{\ell} r}, & \text { if } r \leq R_{\ell}^{s}, \\
q_{m}+\left(h_{\ell}^{s}-q_{m}\right) \mathrm{e}^{\beta_{m}\left(r-R_{\ell}^{s}\right)}, & \text { if } R_{\ell}^{s}<r<R_{g}^{s}, \\
q_{g}+\left(h_{g}^{s}-q_{g}\right) \mathrm{e}^{\beta_{g}\left(r-R_{g}^{s}\right)}, & \text { if } r \geq R_{g}^{s},\end{cases}
\end{gathered}
$$

where

$$
R_{\ell}^{s} \stackrel{\text { def }}{=} \frac{1}{\beta_{\ell}} \ln \left(\frac{h_{\ell}^{s}-q_{\ell}}{\mathcal{H}-q_{\ell}}\right), \quad \quad R_{g}^{s} \stackrel{\text { def }}{=} R_{\ell}^{s}+\frac{1}{\beta_{m}} \ln \left(\frac{h_{g}^{s}-q_{m}}{h_{\ell}^{s}-q_{m}}\right) .
$$

Strategy (5.4) is named INTMOC. We recall that $h_{\kappa}^{s}>q_{\kappa}$ and $h_{g}^{s}>h_{\ell}^{s}>q_{m}-$ see $(3.15)-$ and that $\mathcal{H}>q_{\ell}$ under Hypothesis 4.1. Thus, $R_{\ell}^{s}$ and $R_{g}^{s}$ are well-defined.

Strategies (5.2) and (5.4) being set up, it remains to specify how to compute $\xi_{i}^{n}$ and $\tilde{h}_{i}^{n+1, n}$. These computations are the core of numerical methods of characteristics and are detailed in the next section. 


\subsection{Description of the scheme}

Given the numerical solutions $\left(h_{i}^{n}, v_{i}^{n}, \bar{p}_{i}^{n}\right)$, the overall process at step $n+1$ consists in computing successively $h_{i}^{n+1}, v_{i}^{n+1}$ and $\bar{p}_{i}^{n+1}$ as follows.

Enthalpy: For the boundary condition $(i=1)$ we impose $h_{1}^{n+1}=h_{e}\left(t^{n+1}\right)$. Then $h_{i}^{n+1}$ is determined in two steps:

(1) Solve ODE (5.1b) over the interval $\left[t^{n}, t^{n+1}\right]$. The approximation $\xi_{i}^{n}$ of $\chi\left(t^{n} ; t^{n+1}, y_{i}\right)$ is computed either at order 1 or 2 :

(i) at order 1 in time, we have $\chi\left(t^{n} ; t^{n+1}, y_{i}\right) \approx y_{i}-\Delta t \cdot v\left(t^{n}, y_{i}\right)$ so that we set

$$
\xi_{i}^{n}=y_{i}-\Delta t \cdot v_{i}^{n}
$$

(ii) at order 2 in time (see $[38,39]$ for more details), we have

$$
\chi\left(t^{n} ; t^{n+1}, y_{i}\right) \approx y_{i}-\Delta t \cdot v\left(t^{n}, y_{i}\right)-\frac{1}{2} \Delta t^{2}\left(\partial_{t} v\left(t^{n}, y_{i}\right)-v\left(t^{n}, y_{i}\right) \partial_{y} v\left(t^{n}, y_{i}\right)\right) .
$$

Due to (2.1a) and with a standard 1st-order finite-difference discretization for $\partial_{t} v$, we set

$$
\xi_{i}^{n}=y_{i}-\Delta t\left(\frac{3}{2} v_{i}^{n}-\frac{1}{2} v_{i}^{n-1}\right)+\frac{\Delta t^{2}}{2} \frac{\beta\left(h_{i}^{n}\right)}{p_{0}} v_{i}^{n} \Phi\left(t^{n}, y_{i}\right) .
$$

(2) Update the enthalpy:

- if $\xi_{i}^{n}>0$ (see Fig. 7A), let $j$ be the index such that $\xi_{i}^{n} \in\left[y_{j}, y_{j+1}\right)$ and $\theta_{i j}^{n} \stackrel{\text { def }}{=}\left(y_{j+1}-\xi_{i}^{n}\right) / \Delta y$. As $\xi_{i}^{n}$ is generally not a mesh node, we use an interpolation procedure to evaluate $\tilde{h}_{i}^{n+1, n}$ :

(i) at order 1

$$
\tilde{h}_{i}^{n+1, n}=\theta_{i j}^{n} h_{j}^{n}+\left(1-\theta_{i j}^{n}\right) h_{j+1}^{n} ;
$$

(ii) at higher order (see [38] for more details)

$$
\tilde{h}_{i}^{n+1, n}=\lambda_{i}^{n} h_{j}^{-}+\left(1-\lambda_{i}^{n}\right) h_{j}^{+}
$$

where

$$
\begin{aligned}
& \lambda_{i}^{n} \stackrel{\text { def }}{=}\left\{\begin{array}{lll}
\frac{1+\theta_{i j}^{n}}{3}, & \text { if } \mathcal{P}_{j}^{+}\left(\theta_{i j}^{n}\right) \geq 0 & \text { and } \mathcal{P}_{j}^{-}\left(\theta_{i j}^{n}\right) \geq 0, \\
0, & \text { if } \mathcal{P}_{j}^{+}\left(\theta_{i j}^{n}\right) \geq 0 & \text { and } \mathcal{P}_{j}^{-}\left(\theta_{i j}^{n}\right)<0, \\
1, & \text { if } \mathcal{P}_{j}^{+}\left(\theta_{i j}^{n}\right)<0 & \text { and } \mathcal{P}_{j}^{-}\left(\theta_{i j}^{n}\right) \geq 0, \\
\theta_{i j}^{n}, & \text { otherwise, } &
\end{array}\right. \\
& h_{j}^{-} \stackrel{\text { def }}{=}\left\{\begin{array}{l}
h_{j}^{n}, \quad \text { if } \mathcal{P}_{j}^{+}\left(\theta_{i j}^{n}\right)<0 \text { and } \mathcal{P}_{j}^{-}\left(\theta_{i j}^{n}\right)<0, \\
\frac{\left(\theta_{i j}^{n}\right)^{2}}{2}\left(h_{j-1}^{n}-2 h_{j}^{n}+h_{j+1}^{n}\right)-\frac{\theta_{i j}^{n}}{2}\left(h_{j-1}^{n}-4 h_{j}^{n}+3 h_{j+1}^{n}\right)+h_{j+1}^{n}, \\
\text { otherwise, }
\end{array}\right. \\
& h_{j}^{+} \stackrel{\text { def }}{=}\left\{\begin{array}{l}
h_{j+1}^{n}, \quad \text { if } \mathcal{P}_{j}^{+}\left(\theta_{i j}^{n}\right)<0 \text { and } \mathcal{P}_{j}^{-}\left(\theta_{i j}^{n}\right)<0, \\
\frac{\left(\theta_{i j}^{n}\right)^{2}}{2}\left(h_{j+2}^{n}-2 h_{j+1}^{n}+h_{j}^{n}\right)-\frac{\theta_{i j}^{n}}{2}\left(h_{j+2}^{n}-h_{j}^{n}\right)+h_{j+1}^{n},
\end{array}\right.
\end{aligned}
$$

and $\mathcal{P}_{j}^{ \pm}(\theta) \stackrel{\text { def }}{=}\left(\theta-\delta_{j}^{ \pm}\right)\left(\theta-\delta_{j+1}^{ \pm}\right)$with

$$
\begin{array}{rrr}
\delta_{j}^{-} \stackrel{\text { def }}{=} \frac{2\left(h_{j+1}^{n}-h_{j}^{n}\right)}{h_{j-1}^{n}-2 h_{j}^{n}+h_{j+1}^{n}}, & \delta_{j}^{+} \stackrel{\text { def }}{=} \frac{2\left(h_{j+1}^{n}-h_{j}^{n}\right)}{h_{j}^{n}-2 h_{j+1}^{n}+h_{j+2}^{n}}, \\
\delta_{j+1}^{-} \stackrel{\text { def }}{=} \frac{h_{j-1}^{n}-4 h_{j}^{n}+3 h_{j+1}^{n}}{h_{j-1}^{n}-2 h_{j}^{n}+h_{j+1}^{n}}, & \delta_{j+1}^{+} \stackrel{\text { def }}{=} \frac{h_{j+2}^{n}-h_{j}^{n}}{h_{j}^{n}-2 h_{j+1}^{n}+h_{j+2}^{n}} .
\end{array}
$$




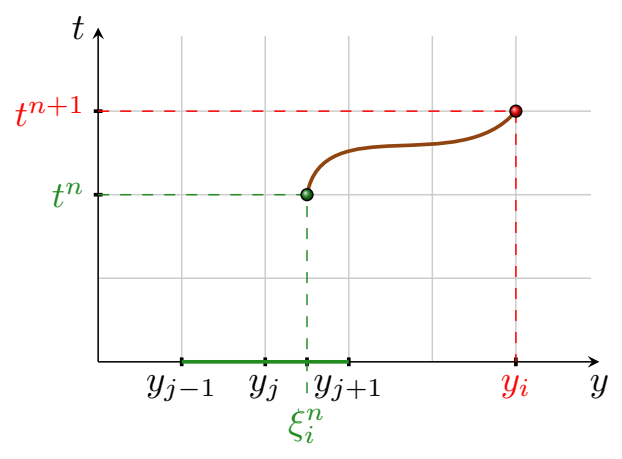

(A) $\xi_{i}^{n}>0$

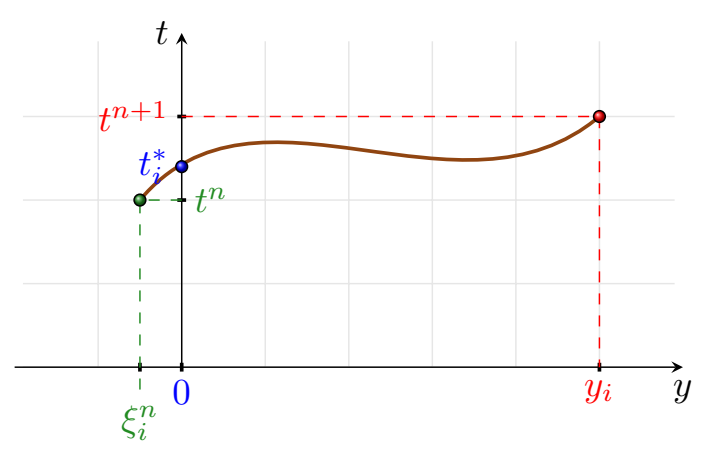

(в) $\xi_{i}^{n} \leq 0$

Figure 7. Numerical method of characteristics.

This procedure has been designed in [38] in order to ensure the maximum principle by means of a variable stencil (and an adaptive order). Even if there is no maximum principle associated to equation (3.17), this scheme preserves the property $\tilde{h}_{i}^{n}-q\left(\tilde{h}_{i}^{n}\right)>0$ (see Prop. 5.1).

We then update $h_{i}^{n+1}$ by formulae (5.2) or (5.4).

- if $\xi_{i}^{n} \leq 0$ (see Fig. 7B), we compute the time $t_{i}^{*}$ at which the characteristic curve $\tau \mapsto \chi\left(\tau ; t^{n+1}, y_{i}\right)$ crosses the inflow boundary. There we have $h\left(t_{i}^{*}, 0\right)=h_{e}\left(t_{i}^{*}\right)$. Using a first order approximation in time, we set $t_{i}^{*}=t^{n+1}-y_{i} / v_{i}^{n}$ and we compute the updated enthalpy similarly to what is detailed above:

(i) by integrating ODE (5.1a) over $\left[t_{i}^{*}, t^{n+1}\right]$ (MOC strategy)

$$
h_{i}^{n+1}=h_{e}\left(t_{i}^{*}\right)+\left(t^{n+1}-t_{i}^{*}\right) \frac{\beta\left(h_{e}\left(t_{i}^{*}\right)\right) \Phi\left(t^{*}, 0\right)}{p_{0}}\left[h_{e}\left(t_{i}^{*}\right)-q\left(h_{e}\left(t_{i}^{*}\right)\right)\right] ;
$$

(ii) by integrating ODE (5.3) over $\left[t_{i}^{*}, t^{n+1}\right]$ (INTMOC strategy)

$$
h_{i}^{n+1}=R^{-1}\left(R\left(h_{e}\left(t_{i}^{*}\right)\right)+\frac{t^{n+1}-t_{i}^{*}}{p_{0}} \frac{\Phi\left(t_{i}^{*}, 0\right)+\Phi\left(t^{n+1}, y_{i}\right)}{2}\right) .
$$

The boundary $y=0$ is the only one we need to care about since characteristic curves cannot exit from the domain at $y=L$ (we assumed that $v_{e}>0$ and $\Phi \geq 0$ which implies that $v>0$ ).

Velocity: For the boundary condition $(i=1)$, we set $v_{1}^{n+1}=v_{e}\left(t^{n+1}\right)$. Then, we integrate equation (2.1a) over $\left[y_{i}, y_{i+1}\right]$. Depending on the ability to compute the primitive function of $\Phi$ (and as $\beta$ is piecewise constant), the velocity field can be computed directly

$$
v_{i}^{n+1}=v_{i-1}^{n+1}+\frac{1}{p_{0}} \int_{y_{i-1}}^{y_{i}} \beta\left(h\left(t^{n+1}, z\right)\right) \Phi\left(t^{n+1}, z\right) \mathrm{d} z
$$

or approximated for example by the following upwind approach

$$
v_{i}^{n+1}=v_{i-1}^{n+1}+\frac{\Delta y}{p_{0}} \beta\left(h_{i-1}^{n+1}\right) \Phi\left(t^{n+1}, y_{i-1}\right)
$$

since $h$ is transported by $v_{i}^{n} \geq 0$. However, since the coefficient $\beta$ is discontinuous at phase change points (see Fig. 3C), we have to adapt the previous algorithm in cells where the fluid changes from a phase to the mixture. It is reasonable to suppose that at most a pure phase and a mixture are present within a single cell (never liquid, mixture and steam simultaneously). Then, if $h_{\kappa}^{s} \in\left(h_{i-1}^{n+1}, h_{i}^{n+1}\right)$, let $y^{*}$ be the linear approximation of $y_{\kappa}^{s}$, i.e.

$$
y^{*} \stackrel{\text { def }}{=} y_{i-1}+\Delta y \frac{h_{\kappa}^{s}-h_{i-1}^{n+1}}{h_{i}^{n+1}-h_{i-1}^{n+1}} .
$$


Hence

$$
\int_{y_{i-1}}^{y_{i}} \beta\left(h\left(t^{n+1}, z\right)\right) \Phi\left(t^{n+1}, z\right) \mathrm{d} z=\int_{y_{i-1}}^{y^{*}} \beta\left(h\left(t^{n+1}, z\right)\right) \Phi\left(t^{n+1}, z\right) \mathrm{d} z+\int_{y^{*}}^{y_{i}} \beta\left(h\left(t^{n+1}, z\right)\right) \Phi\left(t^{n+1}, z\right) \mathrm{d} z
$$

or when the primitive function of $\Phi$ is not known

$$
\int_{y_{i-1}}^{y_{i}} \beta\left(h\left(t^{n+1}, z\right)\right) \Phi\left(t^{n+1}, z\right) \mathrm{d} z \approx\left(y^{*}-y_{i-1}\right) \beta\left(h_{i-1}^{n+1}\right) \Phi\left(t^{n+1}, y_{i-1}\right)+\left(y_{i}-y^{*}\right) \beta\left(h_{i}^{n+1}\right) \Phi\left(t^{n+1}, y_{i}\right) .
$$

Pressure: For the boundary condition $(i=N)$, we set $\bar{p}_{N}^{n}=p_{0}$. Then we rewrite equation (2.1c) in the following equivalent form

$$
\begin{aligned}
-\partial_{y} \bar{p} & =\partial_{t}(\rho(h) v)+\partial_{y}\left(\rho(h) v^{2}\right)-\partial_{y}\left(\mu \partial_{y} v\right)+\rho(h) g \\
& =\rho(h) \partial_{t} v+\rho(h) v \partial_{y} v-\partial_{y}\left(\mu \partial_{y} v\right)+\rho(h) g
\end{aligned}
$$

Using (2.1a) it becomes

$$
-\partial_{y} \bar{p}=\rho(h) \partial_{t} v+\rho(h) v \frac{\beta(h) \Phi}{p_{0}}-\partial_{y}\left(\mu \frac{\beta(h) \Phi}{p_{0}}\right)+\rho(h) g .
$$

Let us note $\rho_{i}^{n+1}=\rho\left(h_{i}^{n+1}\right)$ and $\beta_{i}^{n+1}=\beta\left(h_{i}^{n+1}\right)$. Integrating this equation over $\left[y_{i-1}, y_{i}\right]$, we obtain

$$
\begin{aligned}
\bar{p}_{i-1}^{n+1}= & \bar{p}_{i}^{n+1}+\frac{\Delta y}{2}\left[\left(\rho_{i}^{n+1}+\rho_{i-1}^{n+1}\right) g+\rho_{i}^{n+1} \frac{v_{i}^{n+1}-v_{i}^{n}}{\Delta t}+\rho_{i-1}^{n+1} \frac{v_{i-1}^{n+1}-v_{i-1}^{n}}{\Delta t}\right. \\
& \left.+\rho_{i}^{n+1} v_{i}^{n+1} \frac{\beta_{i}^{n+1}}{p_{0}} \Phi\left(t^{n+1}, y_{i}\right)+\rho_{i-1}^{n+1} v_{i-1}^{n+1} \frac{\beta_{i-1}^{n+1}}{p_{0}} \Phi\left(t^{n+1}, y_{i-1}\right)\right] \\
& -\mu\left[\frac{\beta_{i}^{n+1}}{p_{0}} \Phi\left(t^{n+1}, y_{i}\right)-\frac{\beta_{i-1}^{n+1}}{p_{0}} \Phi\left(t^{n+1}, y_{i-1}\right)\right], \quad i \in\{2, \ldots, N\} .
\end{aligned}
$$

\subsection{Positivity-preserving property}

We have the following result which is the discrete version of Lemma 4.2:

Proposition 5.1. When Hypothesis 4.1 is satisfied, strategies (5.2) and (5.9a) and (5.4) and (5.9b) with wellprepared initial conditions ensure the positivity of $h_{i}^{n}-q\left(h_{i}^{n}\right)$ for any $\Delta t>0$.

Proof. For the sake of clarity, we set $\kappa(h)$ the phase corresponding to the value of $h$, namely $\kappa(h)=\ell$ if $h<h_{\ell}^{s}$, $m$ if $h \in\left[h_{\ell}^{s}, h_{g}^{s}\right]$ and $g$ if $h>h_{g}^{s}$. Let us show by induction the inequalities

$$
h_{i}^{n} \geq \mathcal{H} \quad \text { and } \quad h_{i}^{n}>q_{\kappa\left(h_{i}^{n}\right)} .
$$

We recall that $\mathcal{H}$ is defined within Hypothesis 4.1. Given the physical framework, we assume without loss of generality that the fluid is under the liquid phase initially and at the entry, which means $h_{e}(t)<h_{\ell}^{s}$ and $h_{0}(y)<h_{\ell}^{s}$ for all $t \geq 0$ and $y \in(0, L)$. Estimates (5.13) is satisfied for $n=0$ by hypothesis. If it is satisfied at iteration $n$, then $\tilde{h}_{i}^{n+1, n} \geq \mathcal{H}$ according to the maximum principle verified by the interpolation processes (5.7) (by convexity) and (5.8) (by construction: see [38]). Then, if $\kappa\left(\tilde{h}_{i}^{n+1, n}\right) \in\{m, g\}$, we directly have $\tilde{h}_{i}^{n+1, n}>q_{\kappa\left(\tilde{h}_{i}^{n+1, n}\right)}$ due to (3.15). If $\kappa\left(\tilde{h}_{i}^{n+1, n}\right)=\ell$, then $\tilde{h}_{i}^{n+1, n} \geq \mathcal{H}>q_{\ell}$ due to Hypothesis 4.1. To summarize, we have $\tilde{h}_{i}^{n+1, n}>q_{\kappa\left(\tilde{h}_{i}^{n+1, n}\right)}$.

To complete the proof, we remark that no matter what scheme is used to compute $h_{i}^{n+1}$ from $\tilde{h}_{i}^{n+1, n}$, the cases $\kappa\left(h_{i}^{n+1}\right) \in\{m, g\}$ are trivially handled due to (3.15). It thus remains to deal with $\kappa\left(h_{i}^{n+1}\right)=\ell$. If $h_{i}^{n+1}$ is computed via the MOC scheme $(5.2)$, as $\tilde{h}_{i}^{n+1, n}>q_{\kappa\left(\tilde{h}_{i}^{n+1, n}\right)}$, we remark that $h_{i}^{n+1}>\tilde{h}_{i}^{n+1, n}$, which implies that $\kappa\left(\tilde{h}_{i}^{n+1, n}\right)=\ell$. Thus, (5.2) yields

$$
h_{i}^{n+1}-q_{\ell}=\left(\tilde{h}_{i}^{n+1, n}-q_{\ell}\right)\left(1+\Delta t \frac{\beta_{\ell} \Phi\left(t^{n}, \xi_{i}^{n}\right)}{p_{0}}\right)>0 .
$$


Likewise, if $h_{i}^{n+1}$ is computed via the INTMOC scheme (5.4), as $R^{-1}$ is monotone-increasing owing to (3.15) and Hypothesis 4.1 , we have $h_{i}^{n+1}>\tilde{h}_{i}^{n+1, n}$, which again implies that $\kappa\left(\tilde{h}_{i}^{n+1, n}\right)=\ell$. Scheme (5.4) then reads

$$
h_{i}^{n+1}-q_{\ell}=\left(\tilde{h}_{i}^{n+1, n}-q_{\ell}\right) \exp \left[\Delta t \frac{\beta_{\ell}\left(\Phi\left(t^{n}, \xi_{i}^{n}\right)+\Phi\left(t^{n+1}, y_{i}\right)\right)}{2 p_{0}}\right] \text {. }
$$

Hence the conclusion, which also holds in the exiting characteristic curve case via schemes (5.9a) and (5.9b). Both schemes are thus compatible with our modelling of thermodynamics.

Both schemes (MOC and INTMOC) are explicit and unconditionally stable which are standard features for numerical methods of characteristics. The handling of boundary conditions is achieved in the present study at order 1. Although the time step can be chosen independently from the mesh size, it must be small enough to provide accurate transient results.

\section{NumERICAL EXAMPLES}

In this section, we focus on data sets for which phase transition occurs (for the simulations of single-phase flows, we refer to [6]).

To simulate some scenarii in a PWR, parameters are set as follows:

- Geometry of the reactor: $L=4.2 \mathrm{~m}$;

- Discretization parameters: $N=100$ mesh nodes $(\Delta y \approx 4.3 \mathrm{~cm})$ and $\Delta t=0.01 \mathrm{~s}$;

- Reference value for the pressure, the power density and the velocity: $p_{0}=155 \times 10^{5} \mathrm{~Pa}, \Phi_{0}=$ $170 \times 10^{6} \mathrm{~W} \cdot \mathrm{m}^{-3}, \tilde{v}=0.5 \mathrm{~m} \cdot \mathrm{s}^{-1}$

- Initial data: $h_{0}(y)=h_{e}, v_{0}(y)=v_{e}+\int_{0}^{y} \beta\left(h_{0}(z)\right) \Phi(0, z) / p_{0} \mathrm{~d} z$ (well-prepared insofar as they satisfy the divergence constraint (2.1a));

- Constant viscosity: $\mu_{0}=8.4 \cdot 10^{-5} \mathrm{~kg} \cdot \mathrm{m}^{-1} \cdot \mathrm{s}^{-1}$.

To perform simulations, we have to provide physical values for liquid and vapor stiffened gas parameters. This is achieved by using the tables of water and steam [33] as described in the Appendix C. Parameters involved in the stiffened gas law for the following simulations are those in Table 1. For this data set, variables at saturation are $h_{\ell}^{s} \approx 1.627 \times 10^{6} \mathrm{~J} \cdot \mathrm{K}^{-1}, h_{g}^{s} \approx 3.004 \times 10^{6} \mathrm{~J} \cdot \mathrm{K}^{-1}, \rho_{\ell}^{s} \approx 632.663 \mathrm{~kg} \cdot \mathrm{m}^{-3}$ and $\rho_{g}^{s} \approx 52.937 \mathrm{~kg} \cdot \mathrm{m}^{-3}$.

In Test 6.1, a two-phase flow with phase transition is considered and enables to compare the numerical schemes presented in Section 5, while all other tests only involve scheme (5.4) and (5.9b) with high order interpolation (5.8) (noted INTMOC_2) to assess the robustness of the scheme and the relevance of the model.

\subsection{Two-phase flow with phase transition}

In the first test, we investigate the ability of our model to deal with two-phase flows with phase transition. We consider the case when the inlet density $\rho_{e}$, the inlet velocity $v_{e}$, the initial condition $h_{0}$ and the power density $\Phi$ are constant, so that we can apply Proposition 4.10 to compute exact transient and asymptotic solutions. The boundary conditions are $\rho_{e}(t)=750 \mathrm{~kg} \cdot \mathrm{m}^{-3}$ and $v_{e}(t)=\tilde{v}$, thus $h_{0}(y)=h_{e}(t)=h_{\ell}\left(\rho_{e}\right) \approx 1.190 \times 10^{6} \mathrm{~J} \cdot \mathrm{K}^{-1}$. The power density is set constant in space and time and equal to $\Phi_{0}$. With these parameters, the domain is initially filled with liquid. Then according to Proposition 4.10 mixture appears at time $t_{\ell}^{s} \simeq 1.769 \mathrm{~s}$ for $y>y_{\ell}^{s} \simeq 0.964 \mathrm{~m}$ and pure vapor appears at time $t_{g}^{s} \simeq 2.929 \mathrm{~s}$ for $y>y_{g}^{s} \simeq 4.002 \mathrm{~m}$. The asymptotic state is reached at $t_{g}(L) \simeq 2.957 \mathrm{~s}$.

Figure 8 displays numerical results for the enthalpy and the velocity at instants $t=2.1 \mathrm{~s}, t=2.8 \mathrm{~s}$ and $t=3.5 \mathrm{~s}$. At this last time the solution has already reached the asymptotic regime. Figure 9 displays the mass fraction and the Mach number computed from the enthalpy and the velocity. Figure 10 displays the density and the temperature computed from the enthalpy.

In those figures we compare the exact and asymptotic solutions to the different versions of the numerical scheme, namely:

- by scheme (5.2) and (5.9a) with linear interpolation (5.7) for $\tilde{h}_{i}^{n+1, n}$ (called MOC_1),

- by scheme (5.2) and (5.9a) with high order interpolation (5.8) for $\tilde{h}_{i}^{n+1, n}$ (called MOC_2),

- by scheme (5.4) and (5.9b) with linear interpolation (5.7) for $\tilde{h}_{i}^{n+1, n}$ (called INTMOC_1), 
- by scheme (5.4) and (5.9b) with high order interpolation (5.8) for $\tilde{h}_{i}^{n+1, n}$ (called INTMOC_2).

We mention that the foot of the characteristic curve $\xi_{i}^{n}$ is always computed by means of the 2nd-order expression (5.6) and the velocity field by means of formulae (5.10) and (5.10').

We observe that all numerical results match the behaviour of the exact solution including phase transition. Nevertheless as it can be noticed at time $t=2.8 \mathrm{~s}$, there is some discrepancy close to the singularity (interface between information coming from the boundary and the inner domain). This can easily be accounted for. Indeed, the scheme relies on an interpolation process which assumes some smoothness for the solution. As it is not smooth at the singularity, the scheme provides a smoothened version of the exact solution. It must be underlined that the higher the degree of interpolation, the more accurate the solution. Moreover, there is a clear advantage of INTMOC versions of the scheme over MOC which is due to the better approximation of the right hand side in the former case. For these reasons, from now on, we will use only the INTMOC_2 scheme which is more accurate.

Figure 9 shows that the Mach number remains lower than 0.05 so that the low Mach number hypothesis of the model is valid in this configuration. Observe that during the transient state, the Mach number is greater than at the asymptotic state.

From a physical point of view, it is worth emphasizing that our model predicts the appearance of some vapor at the top of the reactor for this data set. This is due to a too small inflow velocity.

\subsection{Non monotone $\Phi$}

We perform a simulation for a steady power density piecewise constant in space

$$
\Phi(t, y)= \begin{cases}\Phi_{0}, & \text { if } y \leq L / 2 \\ 0, & \text { if } y>L / 2\end{cases}
$$

This power density models the situation where the control rods are blocked at the middle of the reactor. The boundary conditions are $\rho_{e}(t)=750 \mathrm{~kg} \cdot \mathrm{m}^{-3}$ and $v_{e}(t)=\tilde{v}$, thus $h_{0}(y)=h_{e}(t)=h_{\ell}\left(\rho_{e}\right) \approx 1.190 \times 10^{6} \mathrm{~J} \cdot \mathrm{K}^{-1}$. At the initial state the core thus is filled with liquid, so that the void fraction is 0 .

We emphasize that due to the steady boundary condition for the pressure and to the fact that the dynamic pressure is decoupled from the other variables in $1 \mathrm{D}$, the right part $[L / 2, L]$ of the domain does not influence the left part $[0, L / 2]$. This can also be seen in the statement of Proposition 4.10 where the process (for $h$ and $v$ ) evolves from $y=0$ to $y=L$. Hence, we can apply Proposition 4.10 over $[0, L / 2]$ since $\Phi$ is constant within. However, given the solution over $[0, L / 2]$ (and more specifically at $y=L / 2$ ), we cannot apply Proposition 4.10 over $[L / 2, L]$ with these inlet boundary conditions at $y=L / 2$ as they are unsteady until the asymptotic state is reached. Besides, we know the global asymptotic solution which is given by Proposition 4.13.

We plot on Figure 11 the asymptotic solution from Proposition 4.13 and the numerical one computed by means of the INTMOC 2 scheme for the enthalpy, the mass fraction, the dynamical pressure, the density and the temperature at the instants $t=0.0 \mathrm{~s}, t=1.0 \mathrm{~s}, t=2.0 \mathrm{~s}, t=3.0 \mathrm{~s}$ and $t=4.0 \mathrm{~s}$. The velocity is given at instants $t<t_{\ell}^{s}, t=1.8 \mathrm{~s}$ and $t=2.0 \mathrm{~s}$. At $t=t_{\ell}^{s} \simeq 1.768 \mathrm{~s}$ mixture appears over $\left[y_{\ell}^{s}, L / 2\right]$ where $y_{\ell}^{s} \simeq 0.964 \mathrm{~m}$. This mixture is transported to fill the domain $y \geq y_{\ell}^{s}$. As long as the domain is filled with liquid, the velocity does not depend on time. When mixture appears, $\beta$ is piecewise constant in the domain so that, according to Proposition 4.10, the velocity increases up to the asymptotic value. The dynamical pressure is computed by scheme (5.12) and decreases towards the asymptotic state.

\subsection{A simplified scenario for a Loss of Flow Accident}

Our model is tested here on an accidental transient regime: a main coolant pump trip which is a Loss Of Flow Accident (LOFA) as at the beginning of the Fukushima accident in reactors 1, 2 and 3. To simulate this scenario, the domain is filled at first with liquid water and we have a normal behaviour of the reactor: the pumps work normally and control rods are in upper position. We impose at the entrance the velocity $v_{e}=10 \tilde{v}$ and the power density is equal to $\Phi_{0}$. These constants are chosen so as to prevent the appearance of mixture. The asymptotic state is reached at $t \simeq 0.8 \mathrm{~s}$.

At time $t_{1}=1.5 \mathrm{~s}$ most of the pumps stop, so that the entrance velocity decreases. At time $t_{2}$ the security system makes control rods drop into the core decreasing abruptly the power density. However, there is still 

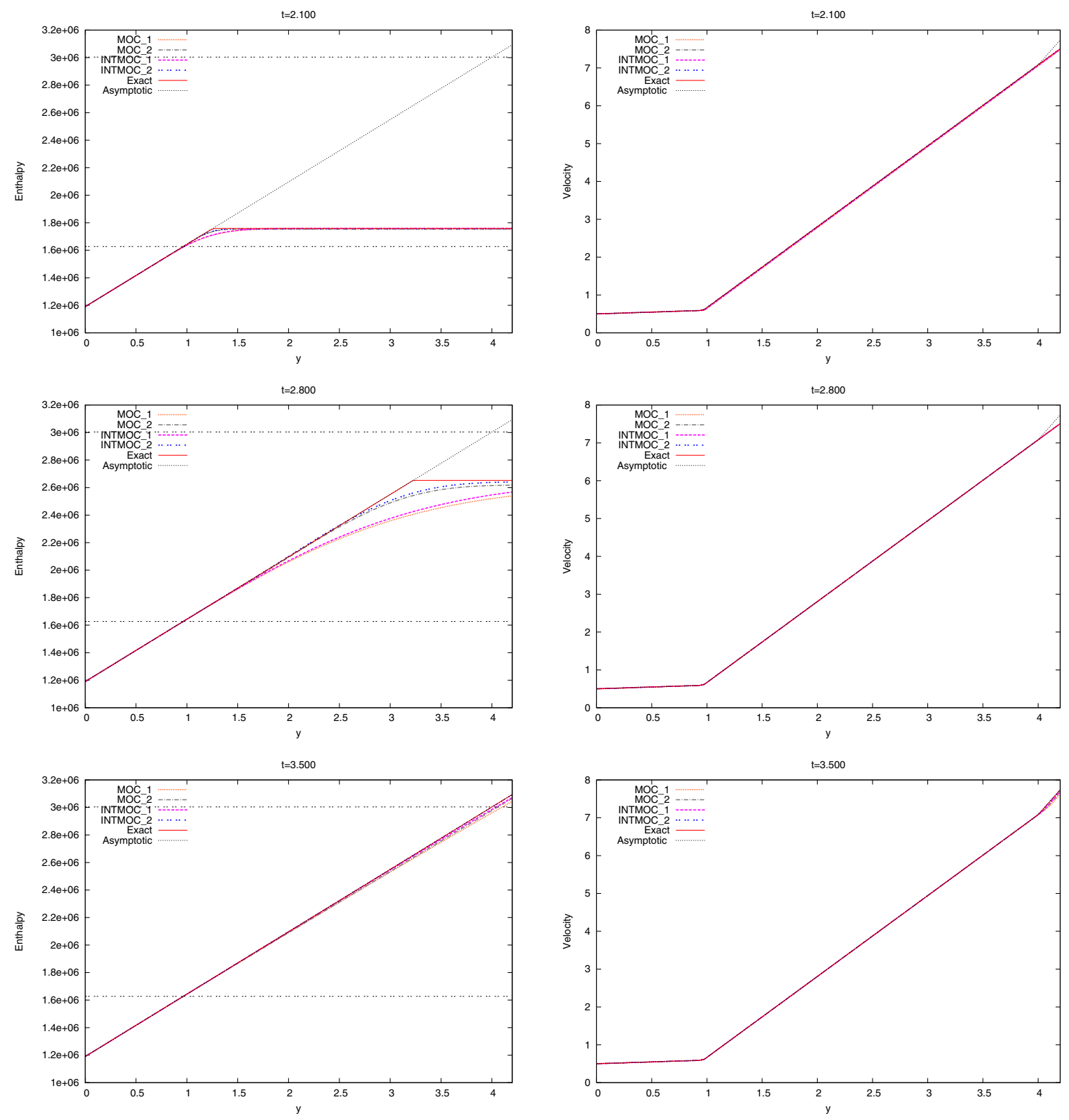

Figure 8. Numerical enthalpy (left) and velocity (right) for test 6.1: Two-phase flow with phase transition. We compare the four numerical solutions to the analytical and asymptotic ones. The horizontal dotted lines correspond to $h=h_{\ell}^{s}$ and $h=h_{g}^{s}$.

some residual power density (here set to $\left.7 \% \Phi_{0}\right)$. This instant $t_{2} \simeq 2.85 \mathrm{~s}$ corresponds to the reaction time $(0.3 \mathrm{~s})$ of the safety system (drop of the control rods) after mixture appears $(t \simeq 2.55 \mathrm{~s})$ and is detected. At time $t_{3}$ the security pumps are turned on, thus the inflow is re-established. We then compute the solution until the asymptotic state is reached. Functions $v_{e}(t)$ and $\Phi(t)$ can be modelled as follows (see Fig. 12):

$$
v_{e}(t)=\left\{\begin{array}{ll}
10 \tilde{v}, & \text { if } 0 \leq t<t_{1}, \\
0.2 \tilde{v}, & \text { if } t_{1} \leq t<t_{3}, \\
10 \tilde{v}, & \text { if } t \geq t_{3},
\end{array} \quad \Phi(t)= \begin{cases}\Phi_{0}, & \text { if } 0 \leq t<t_{2} \\
7 \% \Phi_{0}, & \text { if } t \geq t_{2}\end{cases}\right.
$$



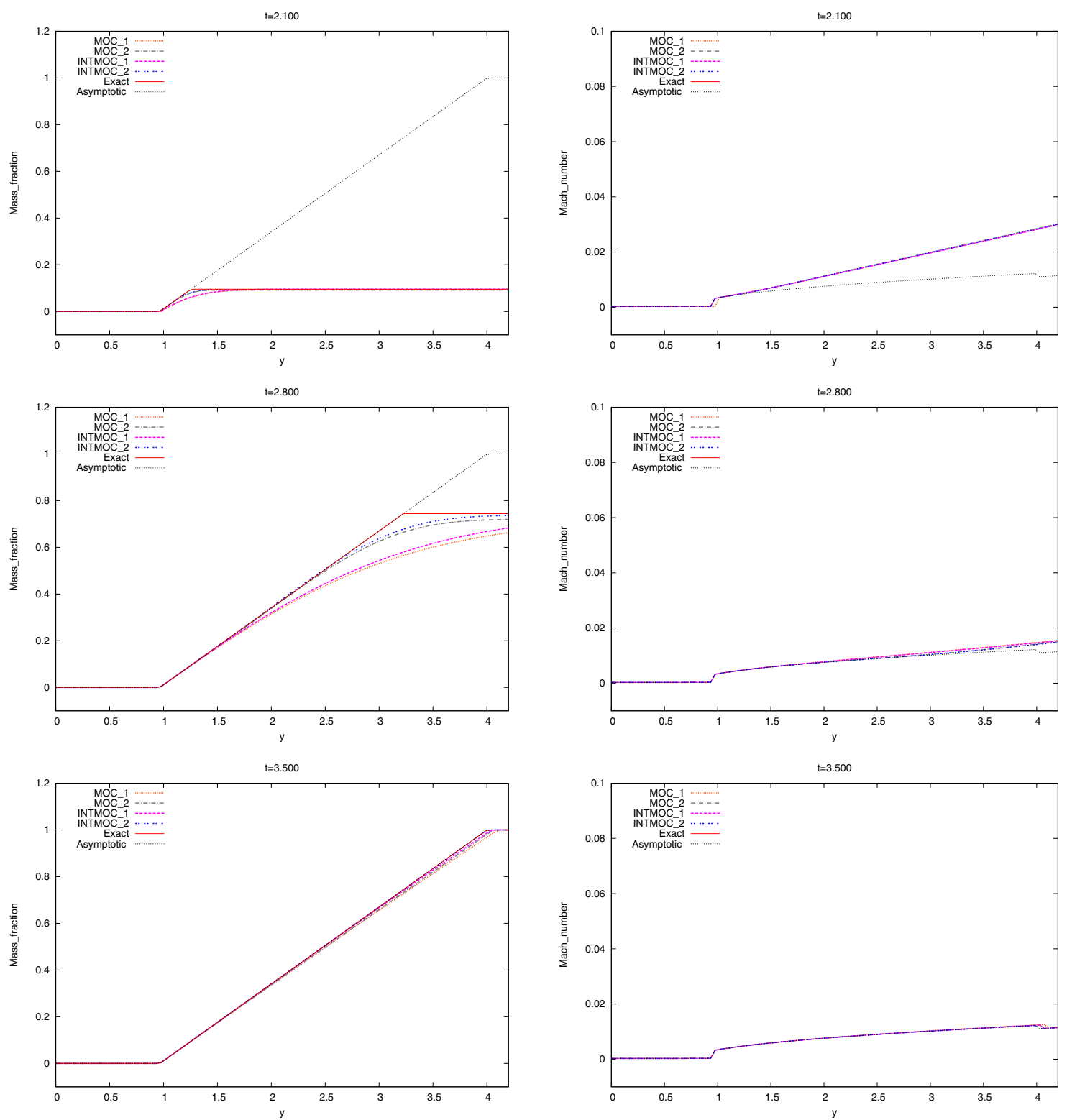

FiguRE 9. Numerical mass fraction (left) and Mach number (right) of the test 6.1: Two-phase flow with phase transition. We compare the four numerical solutions with the analytical and asymptotic ones.

In Figure 13 we report the behaviour of the mass fraction and temperature computed at different instants. Thanks to our model we can predict the appearance of some steam in the core depending of the value of $t_{3}$ :

Case A. for $t_{3}=40 \mathrm{~s}$, the asymptotic state corresponding to $\Phi=7 \% \Phi_{0}$ and $v_{e}=0.2 \tilde{v}$ is established. In this case, due to the residual power density the fluid is completely vaporized at the top of the domain during the transition (see for example Fig. 13A at time $t=30 \mathrm{~s}$ ) even though there are only liquid and mixture phases in the corresponding asymptotic state.

Case B. for $t_{3}=20 \mathrm{~s}$, the pumps are re-started soon enough so that the appearance of pure steam is avoided.

Case C. for $t_{3}=4 \mathrm{~s}$, even though the pumps are re-started almost instantaneously, some mixture appears in a large part of the domain. 

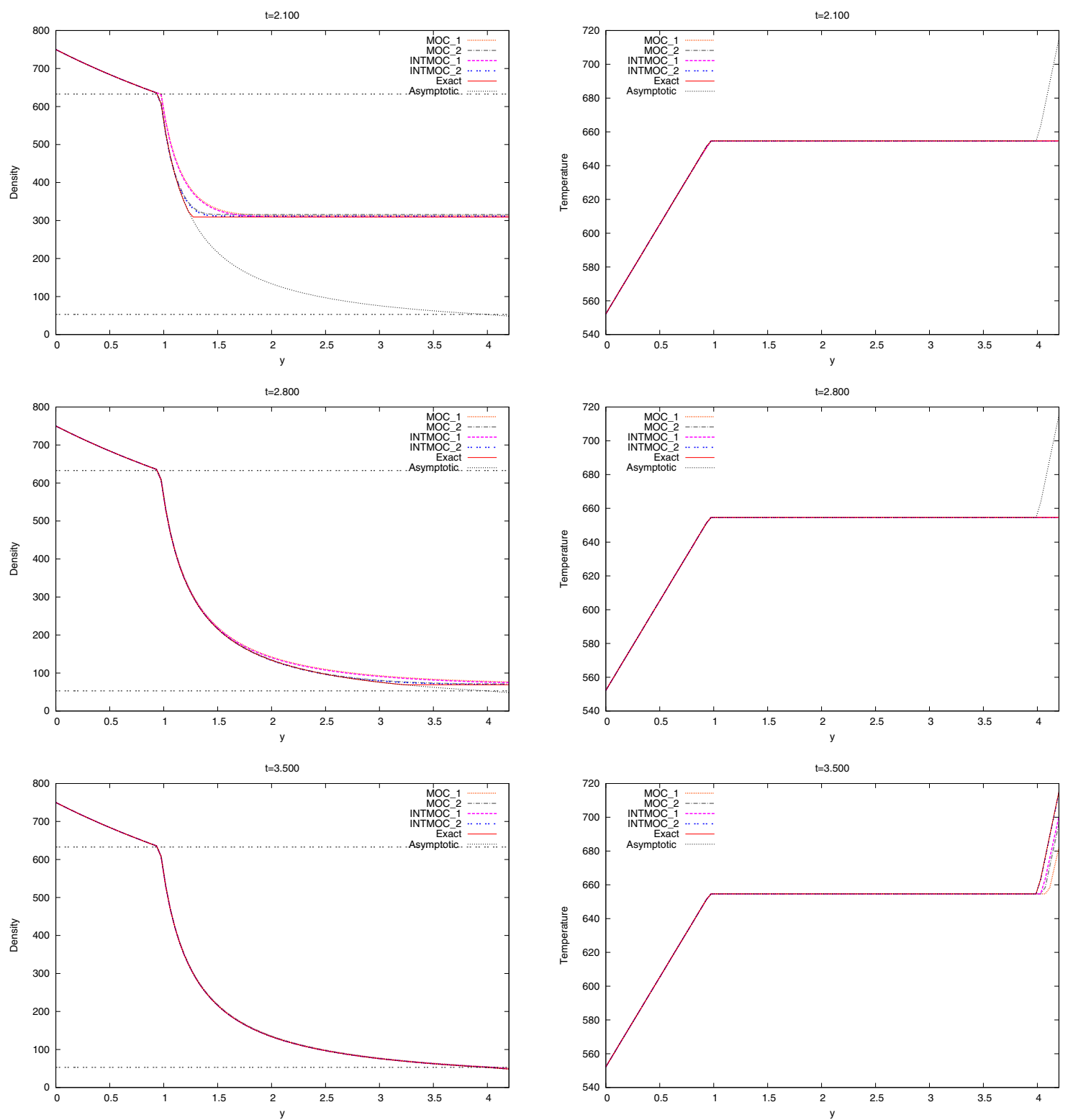

Figure 10. Numerical density (left) and temperature (right) of the test 6.1: Two-phase flow with phase transition. We compare the four numerical solutions with the analytical and asymptotic ones. The horizontal dotted lines correspond to $\rho=\rho_{\ell}^{s}$ and $\rho=\rho_{g}^{s}$.

In all cases when the pumps are re-started, the fluid comes back to the liquid phase. As expected, the sooner the pumps are re-started, the safer the situation.

\section{ConClusion AND PERSPECtives}

We proposed in this paper simulations of a low Mach number model - named LMNC - for fluid flows in nuclear reactor cores coupled to an adaptive stiffened gas equation of state (EOS) which varies according to the phase of the coolant fluid (which can be pure liquid water, pure steam or mixture of these two phases). The monodimensional (1D) numerical strategy we worked out leads to accurate and relevant qualitative results in 


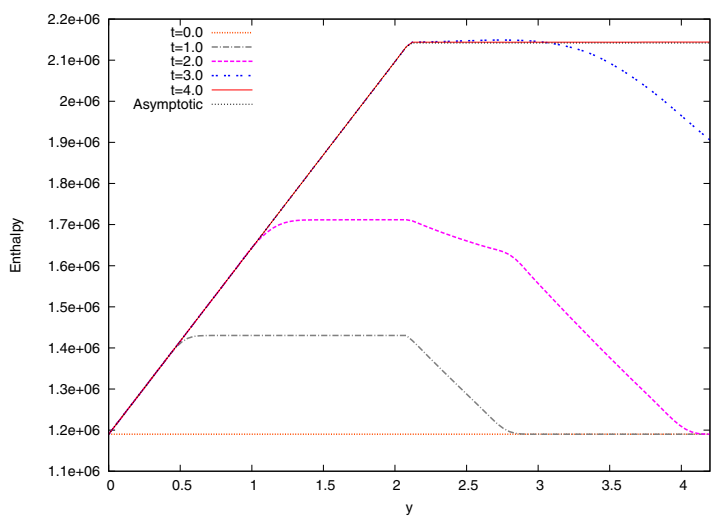

(A) Enthalpy

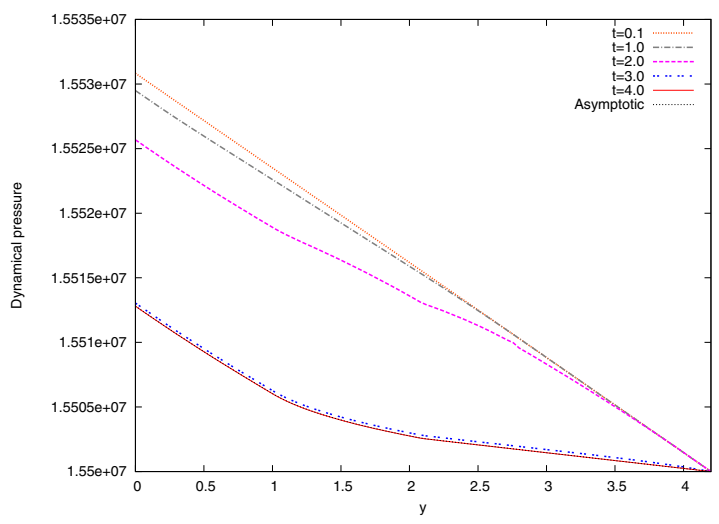

(c) Dynamical pressure

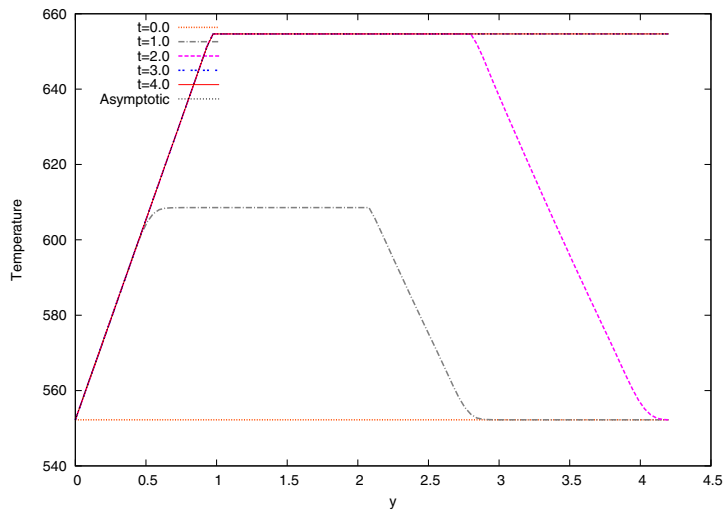

(E) Temperature

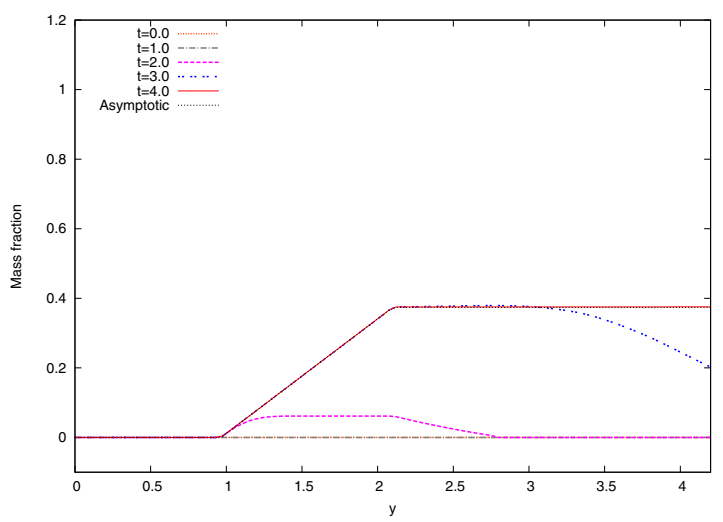

(в) Mass fraction

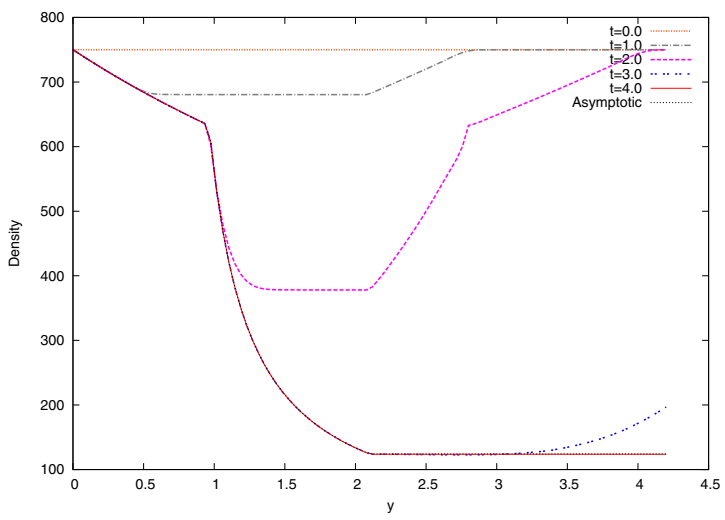

(D) Density

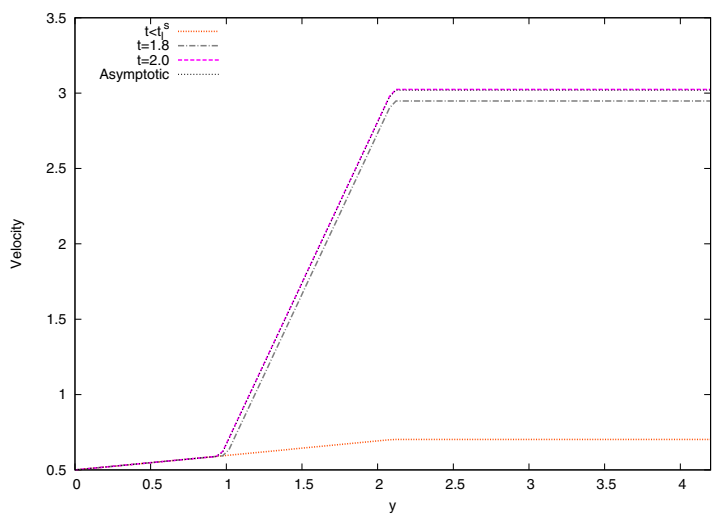

(F) Velocity

FigURE 11. Numerical results of the test 6.2: Non monotone $\Phi$.

accordance with what was expected. These results were obtained with an optimal computational cost since the velocity and the dynamic pressure are directly integrated in the $1 \mathrm{D}$ case.

More precisely, compared to a previous study [6], the present work enables to deal with more realistic situations insofar as the model allows for phase transition and, thus, for accidental scenarii such as a Loss of Flow Accident (LOFA) induced by a coolant pump trip event. Nevertheless, the method used to determine parameters involved in the stiffened gas EOS seems to be restrictive given the range of temperature that must 


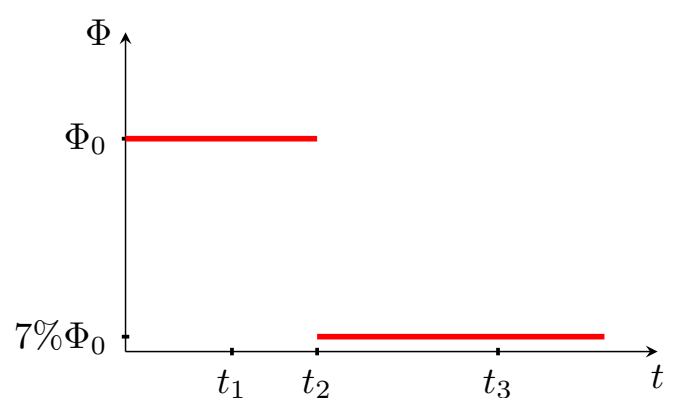

(A) $\Phi(t)$

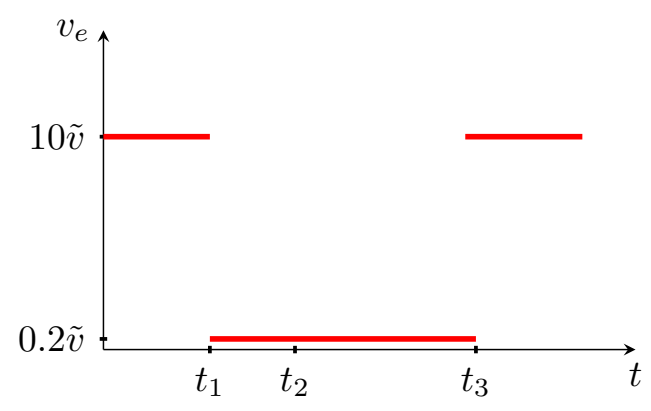

(B) $v_{e}(t)$

Figure 12. Power density and entrance velocity for the test 6.3: A simplified scenario for a Loss of Flow Accident.

be considered. That is why another strategy will be investigated in [18] using tabulated laws instead of the stiffened gas EOS.

From a numerical point of view, a variant of the classic method of characteristics was proposed. It relies on the EOS and provided second-order accurate results. This algorithm was compared to the unsteady analytical solutions derived in this study for different data sets including configurations where phase transition occurs.

Moreover, we recall that the thermodynamic pressure is constant in the LMNC model. Hence, whatever the EOS used to model each phase and no matter what the dimension of the domain $(1 \mathrm{D}, 2 \mathrm{D}$ or $3 \mathrm{D})$, the computational cost related to the EOS will be much lower in the discretization of the LMNC model than in any strategy for the compressible model.

The adaptation of the proposed algorithm to dimensions 2 and 3 will be carried out in future works. However, it must be underlined that although the LMNC model may be a useful tool for safety studies, it is not designed to handle all potential situations especially when the Mach number cannot be considered small anymore. This is why it is important to study the possibility to couple the LMNC model to the compressible system from which it is derived (this approach will be investigated in future works). Likewise, couplings with systems dedicated to other circuits in the reactor must be considered.

\section{Appendix A. Derivation of the miXture Density Formula}

The mechanic and thermodynamic equilibria hypothesis in the mixture at saturation implies that the pressures are identical in each phase. This allows to obtain (3.2) by using (3.1b). This results in the equality for the mixture density $\rho_{m}$

$$
\rho_{m}=\frac{\alpha \rho_{g}^{s} h_{g}^{s}+(1-\alpha) \rho_{\ell}^{s} h_{\ell}^{s}}{h}=\alpha \rho_{g}^{s}+(1-\alpha) \rho_{\ell}^{s}
$$

where $\rho_{\kappa}^{s}$ and $h_{\kappa}^{s}$ are the density and the enthalpy of the phase $\kappa \in\{\ell, g\}$ at saturation, i.e. $\rho_{\kappa}^{s}=\rho_{\kappa}\left(p, T^{s}(p)\right)$ and $h_{\kappa}^{s}=h_{\kappa}\left(p, T^{s}(p)\right)$ where $T^{s}(p)$ is the temperature $T$ solution of $\left.g_{\ell}(p, T)=g_{g}(p, T)\right)$. We deduce $\alpha$ is prescribed by

$$
\alpha(h, p)=\rho_{\ell}^{s} \frac{h-h_{\ell}^{s}}{\left(\rho_{g}^{s} h_{g}^{s}-\rho_{\ell}^{s} h_{\ell}^{s}\right)-h \cdot\left(\rho_{g}^{s}-\rho_{\ell}^{s}\right)} .
$$

Hence we obtain

$$
\rho_{m}(h, p)=\rho_{\ell}^{s}+\alpha(h, p) \cdot\left(\rho_{g}^{s}-\rho_{\ell}^{s}\right)=\frac{\rho_{g}^{s} \rho_{\ell}^{s}\left(h_{g}^{s}-h_{\ell}^{s}\right)}{\rho_{g}^{s} h_{g}^{s}-\rho_{\ell}^{s} h_{\ell}^{s}-h \cdot\left(\rho_{g}^{s}-\rho_{\ell}^{s}\right)},
$$

which gives the expression (3.3) of $\rho_{m}$ as a function of $(h, p)$.

\section{Appendix B. Computation of the speed of Sound for two-Phase Media}

We detail in this section how to express the speed of sound in the diphasic case. This enables in particular to compute the Mach number in any case and to highlight that it remains small. 

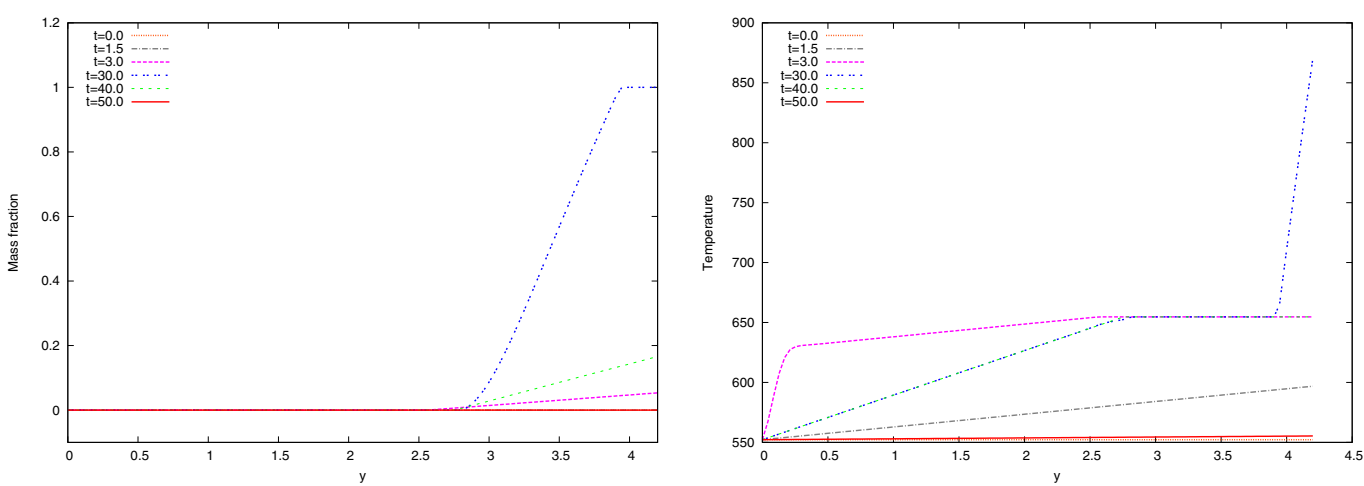

(A) Case A
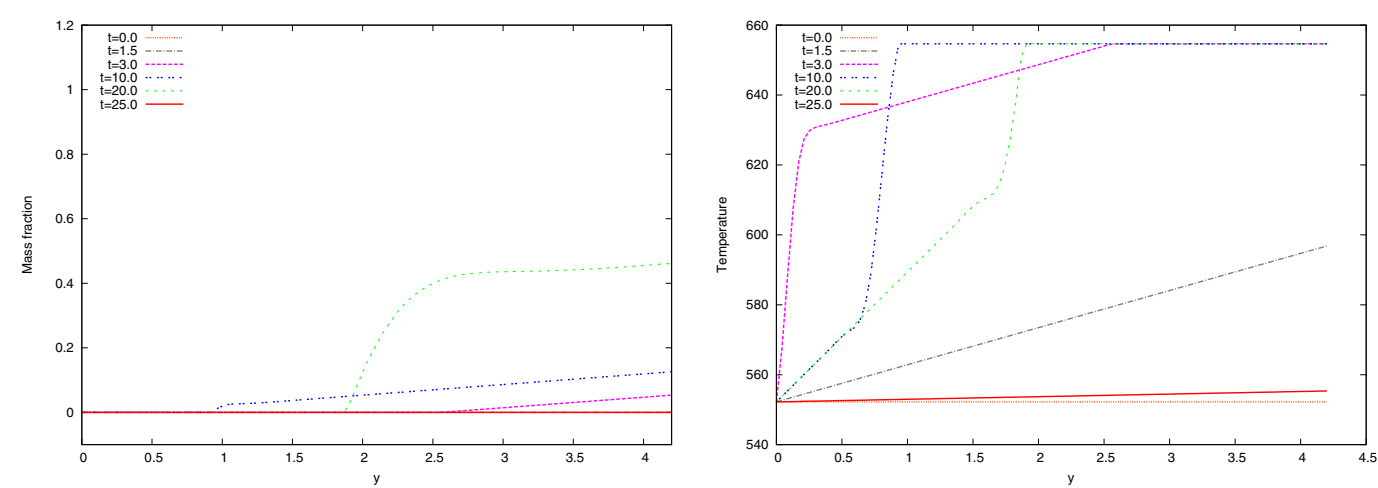

(в) Case B
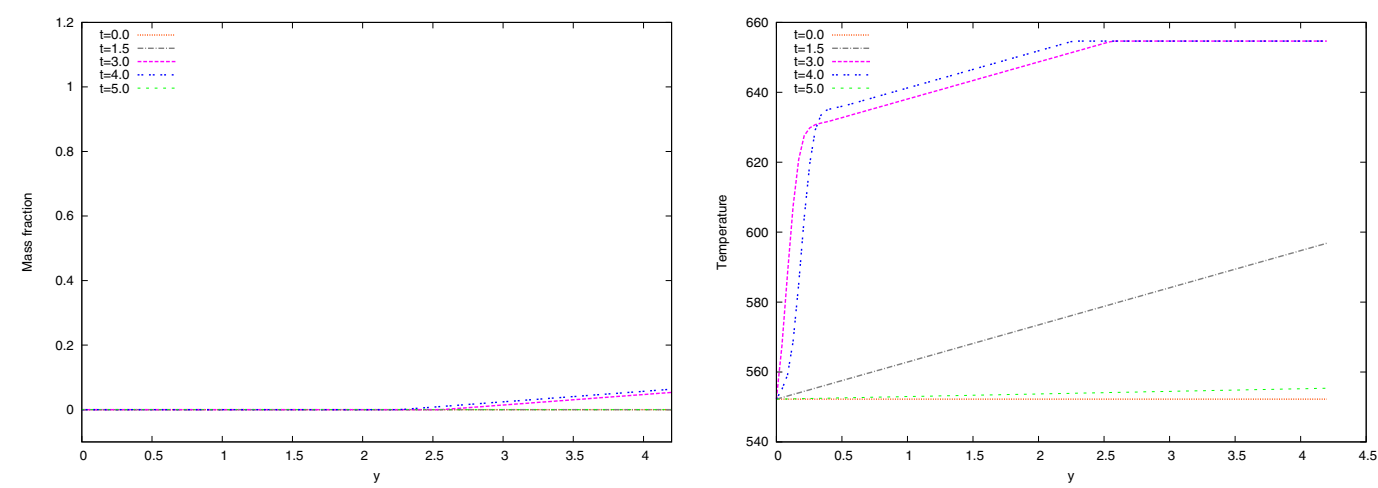

(c) Case C

Figure 13. Numerical mass fraction (left) and temperature (right) of the test 6.3: A simplified scenario for a Loss of Flow Accident.

\section{B.1. General case}

In $[3,22,23]$, it has been proved that the speed of sound of the mixture is always positive. To compute explicitly the speed of sound as a function of the enthalpy and the pressure, we start from the usual thermodynamic relation

$$
T \mathrm{~d} \eta=\mathrm{d} \varepsilon-\frac{p}{\rho^{2}} \mathrm{~d} \rho .
$$

On the one hand, the relation $h=\varepsilon+\frac{p}{\rho}$ coupled to the previous statement leads to

$$
\mathrm{d}(\rho \varepsilon)=(\rho T) \mathrm{d} \eta+h \mathrm{~d} \rho .
$$


On the other hand, we have

$$
\mathrm{d}(\rho \varepsilon)=\left.\frac{\partial(\rho \varepsilon)}{\partial \rho}\right|_{p} \mathrm{~d} \rho+\left.\frac{\partial(\rho \varepsilon)}{\partial p}\right|_{\rho} \mathrm{d} p .
$$

Therefore, the comparison of the two previous equalities yields

$$
\mathrm{d} p=\frac{h-\left.\frac{\partial(\rho \varepsilon)}{\partial \rho}\right|_{p}}{\left.\frac{\partial(\rho \varepsilon)}{\partial p}\right|_{\rho}} \mathrm{d} \rho+\frac{\rho T}{\left.\frac{\partial(\rho \varepsilon)}{\partial p}\right|_{\rho}} \mathrm{d} \eta
$$

so that the speed of sound $c$ is given by

$$
\left.c^{2} \stackrel{\text { def }}{=} \frac{\partial p}{\partial \rho}\right|_{\eta}=\frac{h-\left.\frac{\partial(\rho \varepsilon)}{\partial \rho}\right|_{p}}{\left.\frac{\partial(\rho \varepsilon)}{\partial p}\right|_{\rho}}=\frac{h-\left.\frac{\partial(\rho h)}{\partial \rho}\right|_{p}}{\left.\frac{\partial(\rho h)}{\partial p}\right|_{\rho}-1}
$$

since $\rho \varepsilon=\rho h-p$.

- In the pure phase $\kappa \in\{\ell, g\}$, the volume fraction $\alpha$ is $0\left(i . e . \kappa=\ell\right.$ ) or 1 (i.e. $\kappa=g$ ), so that $\rho=\rho_{\kappa}, h=h_{\kappa}$ and equation (B.1) becomes

$$
c_{\kappa}^{2}=\frac{-\left.\rho_{\kappa} \frac{\partial h_{\kappa}}{\partial \rho_{\kappa}}\right|_{p}}{\left.\rho_{\kappa} \frac{\partial h_{\kappa}}{\partial p}\right|_{\rho_{\kappa}}-1}
$$

- In the mixture, using (3.2) and noticing that $\rho_{\kappa} h_{\kappa}=\rho_{\kappa}^{s} h_{\kappa}^{s}$ (since $T=T^{s}(p)$ in the mixture), which only depends on $p$, we can write

$$
\begin{aligned}
& \left.\frac{\partial(\rho h)}{\partial \rho}\right|_{p}=\left.\frac{\partial \alpha}{\partial \rho}\right|_{p}\left(\rho_{g}^{s} h_{g}^{s}\right)+\left.\alpha \frac{\partial\left(\rho_{g}^{s} h_{g}^{s}\right)}{\partial \rho}\right|_{p}-\left.\frac{\partial \alpha}{\partial \rho}\right|_{p}\left(\rho_{\ell}^{s} h_{\ell}^{s}\right)+\left.(1-\alpha) \frac{\partial\left(\rho_{\ell}^{s} h_{\ell}^{s}\right)}{\partial \rho}\right|_{p}, \\
& \left.\frac{\partial(\rho h)}{\partial p}\right|_{\rho}=\left.\frac{\partial \alpha}{\partial p}\right|_{\rho}\left(\rho_{g}^{s} h_{g}^{s}\right)+\left.\alpha \frac{\partial\left(\rho_{g}^{s} h_{g}^{s}\right)}{\partial p}\right|_{\rho}-\left.\frac{\partial \alpha}{\partial p}\right|_{\rho}\left(\rho_{\ell}^{s} h_{\ell}^{s}\right)+\left.(1-\alpha) \frac{\partial\left(\rho_{\ell}^{s} h_{\ell}^{s}\right)}{\partial p}\right|_{\rho} .
\end{aligned}
$$

Because of (3.1a), we compute the partial derivatives of the volume fraction $\alpha$

$$
\begin{aligned}
& \left.\frac{\partial \alpha}{\partial \rho}\right|_{p}=\frac{1}{\rho_{g}^{s}-\rho_{\ell}^{s}}, \\
& \left.\frac{\partial \alpha}{\partial p}\right|_{\rho}=\frac{-\left(\rho_{\ell}^{s}\right)^{\prime}\left(\rho_{g}^{s}-\rho_{\ell}^{s}\right)-\left(\rho-\rho_{\ell}^{s}\right)\left(\left(\rho_{g}^{s}\right)^{\prime}-\left(\rho_{\ell}^{s}\right)^{\prime}\right)}{\left(\rho_{g}^{s}-\rho_{\ell}^{s}\right)^{2}}=-\frac{\alpha\left(\rho_{g}^{s}\right)^{\prime}+(1-\alpha)\left(\rho_{\ell}^{s}\right)^{\prime}}{\rho_{g}^{s}-\rho_{\ell}^{s}}
\end{aligned}
$$

where $\left(\rho_{\kappa}^{s}\right)^{\prime}$ is the derivative of $p \mapsto \rho_{\kappa}^{s}(p)$. Hence

$$
\left(c_{m}(h, p)\right)^{2} \stackrel{\text { def }}{=} \frac{h-\frac{\rho_{g}^{s} h_{g}^{s}-\rho_{\ell}^{s} h_{\ell}^{s}}{\rho_{g}^{s}-\rho_{\ell}^{s}}}{-\left[\alpha\left(\rho_{g}^{s}\right)^{\prime}+(1-\alpha)\left(\rho_{\ell}^{s}\right)^{\prime}\right] \frac{\rho_{g}^{s} h_{g}^{s}-\rho_{\ell}^{s} h_{\ell}^{s}}{\rho_{g}^{s}-\rho_{\ell}^{s}}+\alpha\left(\rho_{g}^{s} h_{g}^{s}\right)^{\prime}+(1-\alpha)\left(\rho_{\ell}^{s} h_{\ell}^{s}\right)^{\prime}-1},
$$

where $\left(\rho_{\kappa}^{s} h_{\kappa}^{s}\right)^{\prime}$ denotes the derivative of the product $p \mapsto \rho_{\kappa}^{s}(p) h_{\kappa}^{s}(p)$.

Finally, the speed of sound is given by

$$
c(h, p)= \begin{cases}c_{\ell}(h, p), & \text { if } h \leq h_{\ell}^{s}(p) \\ c_{m}(h, p), & \text { if } h_{\ell}^{s}(p)<h<h_{g}^{s}(p), \\ c_{g}(h, p), & \text { if } h \geq h_{g}^{s}(p)\end{cases}
$$

Remark B.1. The speed of sound is discontinuous across the saturation curve, and it is smaller in the mixture than in any pure phase. The minimum value is reached for void ratios close to zero (see [22] and Fig. 3D). 


\section{B.2. Stiffened gas EOS}

To compute the speed of sound $c_{\kappa}$ in a pure phase $(\kappa \in\{\ell, g\})$ using (B.2a), we need to express the enthalpy $h$ as a function of the density $\rho_{\kappa}$ and the pressure $p$. Inverting (3.12a), we obtain

$$
h_{\kappa}(\rho, p)=q_{\kappa}+\frac{\gamma_{\kappa}\left(p+\pi_{\kappa}\right)}{\rho\left(\gamma_{\kappa}-1\right)}
$$

so that (B.2a) becomes

$$
c_{\kappa}(\rho, p)=\sqrt{\frac{\gamma_{\kappa}\left(p+\pi_{\kappa}\right)}{\rho}} .
$$

In the mixture, the speed of sound $c_{m}$ satisfies equation (B.2b) which is written as

$$
\left(c_{m}(h, p)\right)^{2}=\frac{h-q_{m}}{-\left[\alpha\left(\rho_{g}^{s}\right)^{\prime}+(1-\alpha)\left(\rho_{\ell}^{s}\right)^{\prime}\right] q_{m}+\alpha\left(\rho_{g}^{s} h_{g}^{s}\right)^{\prime}+(1-\alpha)\left(\rho_{\ell}^{s} h_{\ell}^{s}\right)^{\prime}-1} .
$$

In this expression, we introduced the following notations

$$
\begin{aligned}
\left(\rho_{\kappa}^{s}\right)^{\prime}(p) & =\frac{1}{\left(\gamma_{\kappa}-1\right) c_{v_{\kappa}} T^{s}(p)}\left(1-\left(p+\pi_{\kappa}\right) \frac{\left(T^{s}\right)^{\prime}}{T^{s}}(p)\right), \\
\left(\rho_{\kappa}^{s} h_{\kappa}^{s}\right)^{\prime}(p) & =\frac{1}{\left(\gamma_{\kappa}-1\right) c_{v_{\kappa}} T^{s}(p)}\left(q_{\kappa}+\gamma_{\kappa} c_{v_{\kappa}} T^{s}(p)-\left(p+\pi_{\kappa}\right) q_{\kappa} \frac{\left(T^{s}\right)^{\prime}}{T^{s}}(p)\right), \\
\left(T^{s}\right)^{\prime}(p) & =\frac{\frac{\left(\gamma_{g}-1\right) c_{v_{g}}}{p+\pi_{g}}-\frac{\left(\gamma_{\ell}-1\right) c_{v_{\ell}}}{p+\pi_{\ell}}}{\left(c_{v_{g}} \gamma_{g}-c_{v_{\ell}} \gamma_{\ell}\right)+\frac{q_{g}-q_{\ell}}{T^{s}(p)}} T^{s}(p)=\frac{\frac{1}{\rho_{g}^{s}(p)}-\frac{1}{\rho_{\ell}^{s}(p)}}{h_{g}^{s}(p)-h_{\ell}^{s}(p)} T^{s}(p)=\frac{T^{s}(p)}{p} \beta_{m}(p) .
\end{aligned}
$$

\section{Appendix C. Approximation of the water EOS With the stiffened gas LaW}

In this appendix, we describe the method introduced in $[31,32,40]$ to choose parameters describing each pure phase of water with a stiffened gas law to best fit with saturated curves (see Fig. 2a). Then, we determine relevant values for liquid water and steam.

More precisely, we suppose that each phase is described by its own stiffened gas EOS (3.7), so that we have to compute five constants for each phase: the specific heat at constant volume $c_{v_{\kappa}}$, the adiabatic coefficient $\gamma_{\kappa}$, the reference pressure $\pi_{\kappa}$, the binding energy $q_{\kappa}$ and the reference entropy $q_{\kappa}^{\prime}$ defined by (3.9). We recall that $\kappa=\ell$ refers to the liquid phase and $\kappa=g$ to the vapor one. These parameters will be determined using experimental values at saturation.

The stiffened gas law consists of the following expressions for the density, the enthalpy and the Gibbs potential as functions of the temperature $T$ and the pressure $p$ :

$$
\begin{aligned}
& \rho_{\kappa}(T, p)=\frac{p+\pi_{\kappa}}{\left(\gamma_{\kappa}-1\right) c_{v_{\kappa}} T}, \\
& \left.h_{\kappa}(T, p)=q_{\kappa}+\gamma_{\kappa} c_{v_{\kappa}} T, \text { (independent of } p\right) \\
& g_{\kappa}(T, p)=q_{\kappa}+T\left[c_{v_{\kappa}} \gamma_{\kappa}-q_{\kappa}^{\prime}-c_{v_{\kappa}} \gamma_{\kappa} \ln T+c_{v_{\kappa}}\left(\gamma_{\kappa}-1\right) \ln \left(p+\pi_{\kappa}\right)\right] .
\end{aligned}
$$

For a given temperature $\bar{T}$, the experimental data needed for the computation are the pressure at saturation $p_{\exp }^{s}(\bar{T})$, the enthalpies at saturation $h_{\kappa, \exp }^{s}(\bar{T})$ and the densities at saturation $\rho_{\kappa, \exp }^{s}(\bar{T})$. We can find these data in [33] for several fluids. Let $T_{0}$ and $T_{1}$ two reference temperatures (recorded in [33]) between the triple point and the critical point (see Fig. 2b). 


\section{Step I. Computation of $\gamma_{\kappa} c_{v_{\kappa}}$.}

From the analytical expression of the enthalpy (C.1b), we have $h_{\kappa}^{\prime}(T)=\gamma_{\kappa} c_{v_{\kappa}}$. This yields an averaged value of the product $c_{p_{\kappa}} \stackrel{\text { def }}{=} \gamma_{\kappa} c_{v_{\kappa}}$ by means of a linear approximation of the experimental enthalpies between reference states $T_{0}$ and $T_{1}$ as

$$
c_{p_{\kappa}}=\frac{h_{\kappa, \exp }^{s}\left(T_{1}\right)-h_{\kappa, \exp }^{s}\left(T_{0}\right)}{T_{1}-T_{0}} .
$$

\section{Step II. Computation of $\boldsymbol{q}_{\boldsymbol{\kappa}}$.}

Equation (C.1b) applied to the reference state $T_{0}$ provides the approximation of the binding energy $q_{\kappa}$ :

$$
q_{\kappa}=h_{\kappa, \exp }^{s}\left(T_{0}\right)-c_{p_{\kappa}} T_{0}
$$

\section{Step III. Computation of $\pi_{\kappa}$.}

At saturation, there is an algebraic relation between the temperature and the pressure, e.g. we can write $p=p^{s}(T)$. Thus, using (C.1a), the specific densities at saturation are expressed as

$$
\rho_{\kappa}^{s}(T)=\rho_{\kappa}\left(T, p^{s}(T)\right)=\frac{p^{s}(T)+\pi_{\kappa}}{\left(c_{p_{\kappa}}-c_{v_{\kappa}}\right) T},
$$

which evaluated at $T_{0}$ and $T_{1}$ yields

$$
\frac{\rho_{\kappa}^{s}\left(T_{1}\right)}{\rho_{\kappa}^{s}\left(T_{0}\right)}=\frac{\left(p^{s}\left(T_{1}\right)+\pi_{\kappa}\right) T_{0}}{\left(p^{s}\left(T_{0}\right)+\pi_{\kappa}\right) T_{1}},
$$

so that we can determine an averaged value of the coefficient $\pi_{\kappa}$ using experimental values of the density and the pressure at saturation

$$
\pi_{\kappa}=\frac{T_{0} \rho_{\kappa, \exp }^{s}\left(T_{0}\right) p_{\exp }^{s}\left(T_{1}\right)-T_{1} \rho_{\kappa, \exp }^{s}\left(T_{1}\right) p_{\exp }^{s}\left(T_{0}\right)}{T_{1} \rho_{\kappa, \exp }^{s}\left(T_{1}\right)-T_{0} \rho_{\kappa, \exp }^{s}\left(T_{0}\right)} .
$$

\section{Step IV. Computation of $\boldsymbol{c}_{\boldsymbol{v}_{\kappa}}$.}

Equation (C.3) applied to the reference state $T_{0}$ provides the approximation of $c_{v_{\kappa}}$

$$
c_{v_{\kappa}}=c_{p_{\kappa}}-\frac{p_{\exp }^{s}\left(T_{0}\right)+\pi_{\kappa}}{T_{0} \rho_{\kappa, \exp }^{s}\left(T_{0}\right)}
$$

\section{Step V. Computation of $\gamma_{\kappa}$.}

Using (C.2) we deduce the approximation of $\gamma_{\kappa}$

$$
\gamma_{\kappa}=\frac{c_{p_{\kappa}}}{c_{v_{\kappa}}}
$$

\section{Step VI. Computation of $q_{\kappa}^{\prime}$.}

At thermodynamic equilibrium, the two Gibbs potentials are equal. Using (C.1c), this implies

$$
A \ln \left(p^{s}(T)+\pi_{g}\right)-B \ln \left(p^{s}(T)+\pi_{\ell}\right)-C(\ln T-1)+\frac{D}{T}+q_{\ell}^{\prime}-q_{g}^{\prime}=0
$$

with

$$
A \stackrel{\text { def }}{=} c_{p_{g}}-c_{v_{g}}, \quad B \stackrel{\text { def }}{=} c_{p_{\ell}}-c_{v_{\ell}}, \quad C \stackrel{\text { def }}{=} c_{p_{g}}-c_{p_{\ell}}, \quad D \stackrel{\text { def }}{=} q_{g}-q_{\ell}
$$

By convention, we take $q_{\ell}^{\prime}=0 \mathrm{~J} \cdot \mathrm{K}^{-1}$ and determine the coefficient $q_{g}^{\prime}$ using experimental values of the pressure at saturation

$$
q_{g}^{\prime}=A \ln \left(p_{\exp }^{s}\left(T_{0}\right)+\pi_{g}\right)-B \ln \left(p_{\exp }^{s}\left(T_{0}\right)+\pi_{\ell}\right)-C\left(\ln T_{0}-1\right)+\frac{D}{T_{0}} .
$$

Reference states $T_{0}$ and $T_{1}$ must now be specified. First, $T_{0}$ is a reference state chosen in order to have the best fit between the theoretical pressure $p^{s}(T)$ and the experimental one $p_{\exp }^{s}(T)$. Secondly, this strategy for 
the determination of parameters for a stiffened gas EOS is accurate if the two reference states are sufficiently close. Practically (following [31,32]), we took for the liquid phase $T_{0}=298 \mathrm{~K}$ and $T_{1}=473 \mathrm{~K}$ (Steps I and II) and $T_{0}=439 \mathrm{~K}$ and $T_{1}=588 \mathrm{~K}$ (Steps III to VI). As for the steam, we set $T_{0}=298 \mathrm{~K}$ and $T_{1}=473 \mathrm{~K}$ in any step.

The values for liquid water and steam are given in Table 1 . As underlined in [22,31,32], these parameter values yield reasonable approximations over a temperature range from $298 \mathrm{~K}$ to $473 \mathrm{~K}$. Nevertheless near the critical point, there are some restrictions due to the nonlinearity of the enthalpy with respect to the temperature. To circumvent this limitation, we shall propose in [18] a new strategy to better approximate the thermodynamic quantities involved in the LMNC model.

\section{REFERENCES}

[1] TRACE V5.0 Theory Manual, Field Equations, Solution Methods and Physical Models. Technical report, U.S. Nuclear Regulatory Commission (2008).

[2] A. Acrivos, Method of characteristics technique. Application to heat and mass transfer problems. Ind. Eng. Chem. 48 (1956) $703-710$.

[3] G. Allaire, G. Faccanoni and S. Kokh, A strictly hyperbolic equilibrium phase transition model. C. R. Acad. Sci. Paris Ser. I 344 (2007) 135-140.

[4] A.S. Almgren, J.B. Bell, C.A. Rendleman and M. Zingale, Low Mach number modeling of type Ia supernovae. I. hydrodynamics. Astrophys. J. 637 (2006) 922.

[5] A.S. Almgren, J.B. Bell, C.A. Rendleman and M. Zingale, Low Mach number modeling of type Ia supernovae. II. energy evolution. Astrophys. J. 649 (2006) 927.

[6] M. Bernard, S. Dellacherie, G. Faccanoni, B. Grec, O. Lafitte, T.-T. Nguyen and Y. Penel. Study of low Mach nuclear core model for single-phase flow. ESAIM Proc. 38 (2012) 118-134.

[7] D. Bestion. The physical closure laws in the CATHARE code. Nucl. Eng. Des. 124 (1990) 229-245.

[8] H. B. Callen, Thermodynamics and an Introduction to Thermostatistics. 2nd edition. John Wiley and sons (1985).

[9] V. Casulli and D. Greenspan, Pressure method for the numerical solution of transient, compressible fluid flows. Int. J. Numer. Methods Fluids 4 (1984) 1001-1012.

[10] S. Clerc, Numerical Simulation of the Homogeneous Equilibrium Model for Two-Phase Flows. J. Comput. Phys. 181 (2002) 577-616.

[11] P. Colella and K. Pao, A projection method for low speed flows. J. Comput. Phys. 149 (1999) 245-269.

[12] J.M. Delhaye, Thermohydraulique des réacteurs. EDP sciences (2008).

[13] S. Dellacherie, On a diphasic low Mach number system. ESAIM: M2AN 39 (2005) 487-514.

[14] S. Dellacherie, Numerical resolution of a potential diphasic low Mach number system. J. Comput. Phys. 223 (2007) $151-187$.

[15] S. Dellacherie, Analysis of Godunov type schemes applied to the compressible Euler system at low Mach number. J. Comput. Phys. 229 (2010) 978-1016.

[16] S. Dellacherie, On a low Mach nuclear core model. ESAIM Proc. 35 (2012) 79-106.

[17] S. Dellacherie, G. Faccanoni, B. Grec, F. Lagoutière, E. Nayir and Y. Penel, 2D numerical simulation of a low Mach nuclear core model with stiffened gas using Freefem++. ESAIM. Proc. (accepted).

[18] S. Dellacherie, G. Faccanoni, B. Grec and Y. Penel, Study of low Mach nuclear core model for two-phase flows with phase transition II: tabulated EOS. In preparation.

[19] M. Drouin, O. Grégoire and O. Simonin, A consistent methodology for the derivation and calibration of a macroscopic turbulence model for flows in porous media. Int. J. Heat Mass Transfer 63 (2013) 401-413.

[20] D.R. Durran, Numerical methods for fluid dynamics, With applications to Geophysics, vol. 32 of Texts in Applied Mathematics. Springer, 2nd edition. New York (2010).

[21] P. Embid, Well-posedness of the nonlinear equations for zero Mach number combustion. Comm. Partial Differ. Equ. 12 (1987) $1227-1283$.

[22] G. Faccanoni, Étude d'un modèle fin de changement de phase liquide-vapeur. Contribution à l'étude de la crise d'ébullition. Ph.D. thesis, École Polytechnique, France (2008)

[23] G. Faccanoni, S. Kokh and G. Allaire, Modelling and simulation of liquid-vapor phase transition in compressible flows based on thermodynamical equilibrium. ESAIM: M2AN 46 1029-1054 2012.

[24] P. Fillion, A. Chanoine, S. Dellacherie and A. Kumbaro, FLICA-OVAP: A new platform for core thermal-hydraulic studies. Nucl. Eng. Des. 241 (2011) 4348-4358.

[25] E. Goncalvès and R.F. Patella, Numerical study of cavitating flows with thermodynamic effect. Comput. Fluids 39 (2010) 99-113.

[26] J.M. Gonzalez-Santalo and R.T. Jr Lahey, An exact solution for flow transients in two-phase systems by the method of characteristics. J. Heat Transfer 95 (1973) 470-476.

[27] W. Greiner, L. Neise and H. Stöcker, Thermodynamics and statistical mechanics. Springer (1997).

[28] H. Guillard and C. Viozat, On the behaviour of upwind schemes in the low Mach number limit. Comput. Fluids 28 (1999) 63-86.

[29] S. Jaouen, Étude mathématique et numérique de stabilité pour des modeles hydrodynamiques avec transition de phase. Ph.D. thesis, Université Paris 6, France (2001).

[30] M.F. Lai, J.B. Bell and P. Colella. A projection method for combustion in the zero Mach number limit, in Proc. of 11th AIAA Comput. Fluid Dyn. Conf. (1993) 776-783. 
[31] O. Le Métayer, J. Massoni and R. Saurel, Elaborating equations of state of a liquid and its vapor for two-phase flow models. Int. J. Therm. Sci. 43 (2004) 265-276,.

[32] O. Le Métayer, J. Massoni and R. Saurel, Modelling evaporation fronts with reactive Riemann solvers. J. Comput. Phys. 205 (2005) 567-610.

[33] E.W. Lemmon, M.O. McLinden and D.G. Friend, Thermophysical Properties of Fluid Systems. National Institute of Standards and Technology, Gaithersburg MD, 20899.

[34] A. Majda and K.G. Lamb, Simplified equations for low Mach number combustion with strong heat release, Dynamical issues in combustion theory, vol. 35 of IMA Vol. Math. Appl. Springer-Verlag (1991).

[35] A. Majda and J. Sethian, The derivation and numerical solution of the equations for zero Mach number combustion. Combust. Sci. Technol. 42 (1985) 185-205.

[36] R. Menikoff and B.J. Plohr, The Riemann problem for fluid flow of real materials. Rev. Modern Phys. 61 (1989) 75-130.

[37] S. Müller and A. Voss, The Riemann problem for the Euler equations with nonconvex and nonsmooth equation of state: construction of wave curves. SIAM J. Sci. Comput. 28 (2006) 651-681.

[38] Y. Penel, An explicit stable numerical scheme for the 1D transport equation. Discrete Contin. Dyn. Syst. Ser. S 5 (2012) 641-656.

[39] Y. Penel, Existence of global solutions to the 1D abstract bubble vibration model. Differ. Integral Equ. 26 (2013) 59-80.

[40] R. Saurel, F. Petitpas and R. Abgrall, Modelling phase transition in metastable liquids: application to cavitating and flashing flows. J. Fluid Mech. 607 (2008) 313-350.

[41] G.I. Sivashinsky, Hydrodynamic theory of flame propagation in an enclosed volume. Acta Astronaut. 6 (1979) $631-645$.

[42] G. Volpe, Performance of compressible flow codes at low Mach numbers. AIAA J. 31 (1993) 49-56.

[43] A. Voss, Exact Riemann solution for the Euler equations with nonconvex and nonsmooth equation of state. Ph.D. thesis, RWTH Aachen (2005).

[44] N. Zuber, Flow excursions and oscillations in boiling, two-phase flow systems with heat addition, in Symposium on Two-phase Flow Dynamics, Eindhoven EUR4288e (1967) 1071-1089. 